\title{
A wholehearted computational assessment of cardiac pacing
}

Citation for published version (APA):

Willemen, E. (2021). A wholehearted computational assessment of cardiac pacing. [Doctoral Thesis, Maastricht University]. Maastricht University. https://doi.org/10.26481/dis.20210521ew

Document status and date:

Published: 01/01/2021

DOI:

10.26481/dis.20210521ew

Document Version:

Publisher's PDF, also known as Version of record

\section{Please check the document version of this publication:}

- A submitted manuscript is the version of the article upon submission and before peer-review. There can be important differences between the submitted version and the official published version of record.

People interested in the research are advised to contact the author for the final version of the publication, or visit the DOI to the publisher's website.

- The final author version and the galley proof are versions of the publication after peer review.

- The final published version features the final layout of the paper including the volume, issue and page numbers.

Link to publication

\footnotetext{
General rights rights.

- You may freely distribute the URL identifying the publication in the public portal. please follow below link for the End User Agreement:

www.umlib.nl/taverne-license

Take down policy

If you believe that this document breaches copyright please contact us at:

repository@maastrichtuniversity.nl

providing details and we will investigate your claim.
}

Copyright and moral rights for the publications made accessible in the public portal are retained by the authors and/or other copyright owners and it is a condition of accessing publications that users recognise and abide by the legal requirements associated with these

- Users may download and print one copy of any publication from the public portal for the purpose of private study or research.

- You may not further distribute the material or use it for any profit-making activity or commercial gain

If the publication is distributed under the terms of Article $25 \mathrm{fa}$ of the Dutch Copyright Act, indicated by the "Taverne" license above, 


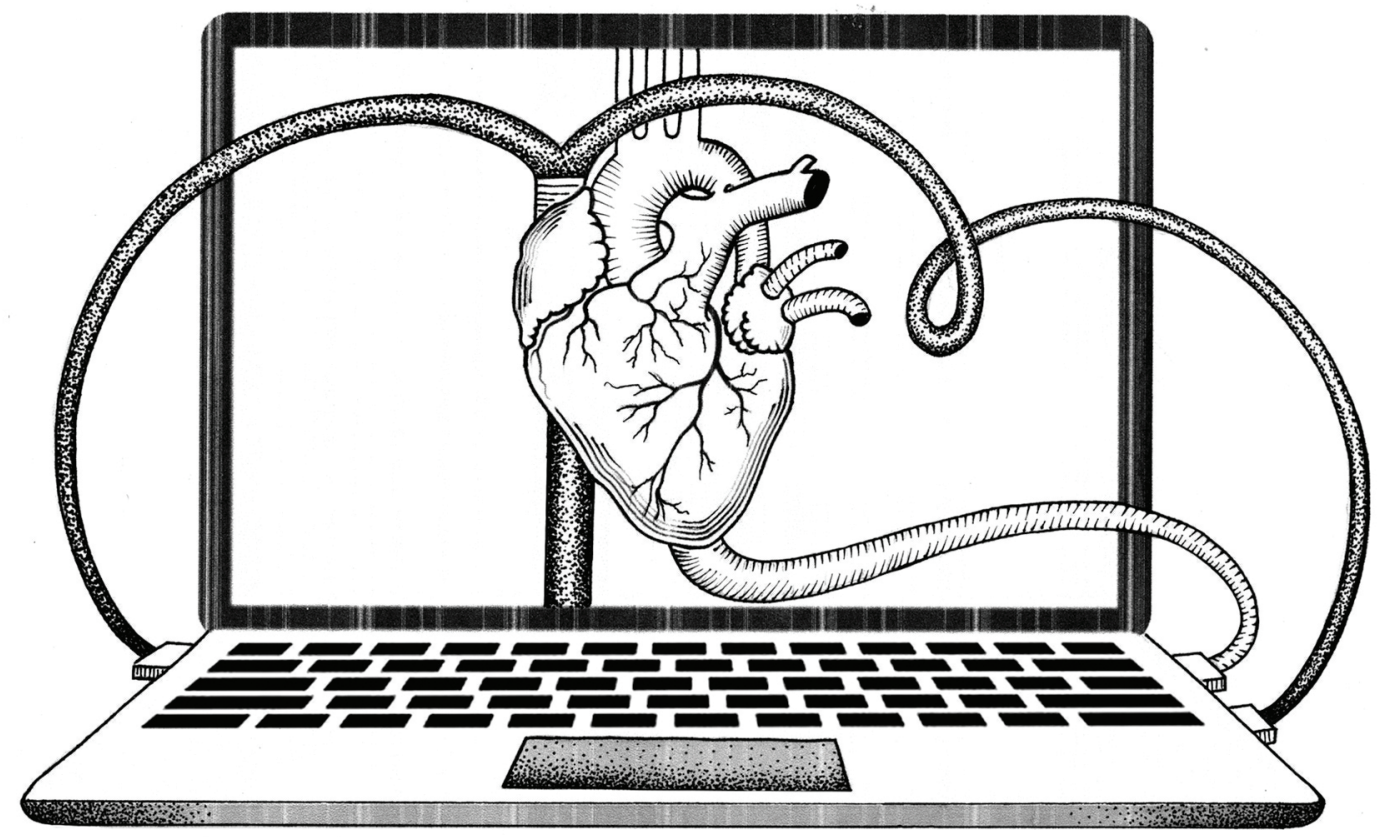

\section{A wholehearted computational assessment of cardiac pacing}

Erik Willemen 
Copyright (c) 2021 by Erik Willemen

Cover \& title page artwork by Luuk Willemen ISBN: 978-94-6423-251-6 


\section{A wholehearted \\ computational assessment of cardiac pacing}

Proefschrift

ter verkrijging van de graad van doctor aan de Universiteit Maastricht, op gezag van de Rector Magnificus, prof. dr. Rianne M. Letschert, volgens het besluit van het College van Decanen,

in het openbaar te verdedigen

op vrijdag 21 mei 2021 om 14:00 uur.

door

Erik Willemen

geboren op 10 januari 1989

te Hengelo (Overijssel), Nederland 


\section{Promotores}

Prof. dr. F.W. Prinzen

Prof. dr. T. Delhaas

\section{Copromotor}

Dr. ir. J. Lumens

\section{Beoordelingscommissie}

Prof. Dr. U. Schotten (voorzitter)

Dr. C.P. Allaart (Amsterdam UMC)

Prof. dr. H.J.G.M. Crijns

Prof. dr. ir. R. Peeters

Dr. ir. B.E. Westerhof (University of Twente)

Parts of the research described in this thesis was funded through the EraCoSysMed PUSHCART project. 


\section{Table of Contents}

Chapter 1 General Introduction

Chapter 2 Importance of effective atrioventricular

delay for ventricular filling and cardiac pump function

Chapter 3 The left and right ventricles respond differently to variation of pacing delays in cardiac resynchronization therapy

Chapter 4 Impact of Paced Left Ventricular

Resynchronization Therapy

Chapter 5 LBBB and High Afterload

Chapter 6 


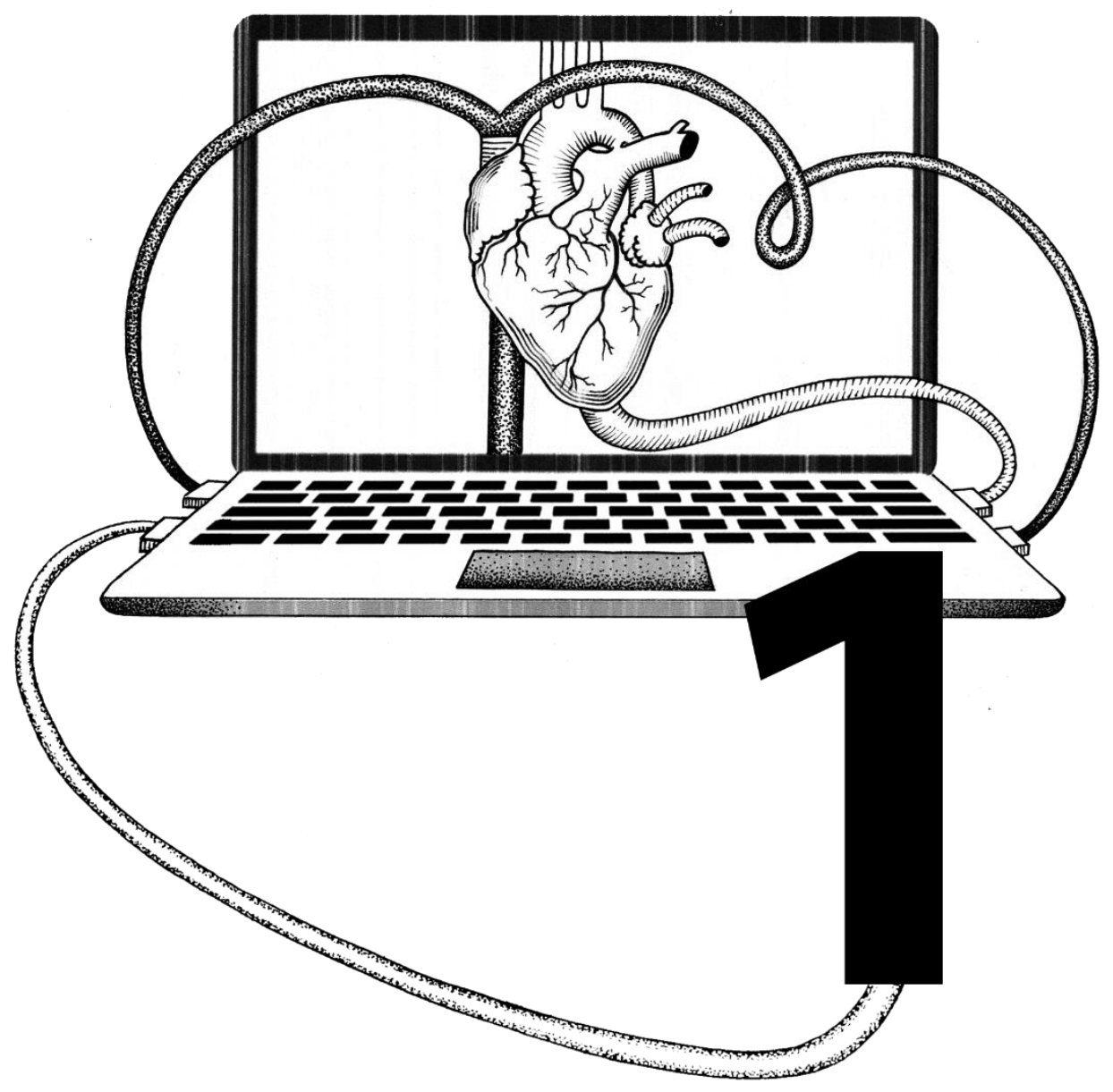


Chapter 1

General introduction 

The pumping action of the healthy human heart ensures circulation of blood through our cardiovascular system. Normal cardiac pump function is by no means self-evident, but requires all four chambers to work together in a continuous and tightly coordinated effort. Cardiac muscle tissue and thereby the heart contracts when it is triggered with an electrical stimulus. In a normal heart cycle the first electrical stimulus originates from the sinoatrial node (SA node), located in the upper part of the right atrium. The resulting electrical wave front travels over, and activates the right and left atrium, and reaches the atrioventricular node ( $A V$ node). In a healthy heart, the AV node is the only location where electrical signals can propagate from the atria to the ventricles. In the AV node the electrical wave front propagation is slower than in cardiac tissue, causing a delay between atrial and ventricular activation. Propagation within the ventricle is fast as a result of the fast conducting His-Purkinje system. Starting from the AV node the electrical wave front travels at high velocity through the bundle of His, down the right and left bundle branches towards the Purkinje fibers. This results in an efficient synchronous contraction because the fast conduction systems allows all segments of the ventricles to contract within a short time ${ }^{1}$.

Disturbances in normal electrical conduction in the heart affect cardiac function. In this thesis we mainly focused on two cardiac conduction disturbances:

1. Blockage of the AV node ( $A V$ block): This leads to electrical uncoupling of atria to ventricles. As a result, the ventricles do not contract frequently enough (ventricular bradycardia) which can lead to insufficient blood flow which in its turn results in syncope and/or organ under perfusion.

2. Blockage of parts of the ventricular rapid conduction system, such as left and right bundle branch: Leading to dyssynchronous ventricular activation and a less efficient contraction. In ventricular dyssynchrony early activated regions start to shorten before aortic 
valve opening, thereby stretching later activated regions. Once these pre-stretched late activated regions are activated, they contract more forcefully due to the local Frank-Starling effect. The stronger contraction causes mid-systolic stretching of the earlier activated regions. Part of the work performed by the ventricle during ventricular dyssynchrony is thus used for stretching ventricular segments activated at another time, instead of building up ventricular pressure. Left ventricular dyssynchrony is associated with a higher risk of heart failure and mortality ${ }^{2,3}$.

When disturbances in electrical conduction lead to acute symptoms or increase in mortality, artificial electrical stimulation of the heart (cardiac pacing) can support or take over a patients' electrical activation. In cardiac pacing, electrode(s) are placed in or on the atria and/or ventricle(s) to provide an electrical stimulus. Cardiac pacing is predominantly, and for already more than 60 years, used in patients with symptomatic bradycardia, including patients with AV block. In these patients, cardiac pacing restores the natural heart rate and cardiac output to provide sufficient blood circulation for organ perfusion. However, conventional RV pacing causes an abnormal, more dyssynchronous activation compared to normal healthy activation. In bradycardic patients with a normal left ventricular (LV) ejection fraction, it was shown that conventional RV pacing can result in remodeling, atrial fibrillation and heart failure ${ }^{4,5}$. Moreover, AV delay is important to create optimal coupling between atria and ventricles.

A newer pacemaker therapy is cardiac resynchronization therapy (CRT). CRT can be applied in patients with ventricular conduction abnormalities like left bundle branch block (LBBB) to restore a more synchronous activation by pacing both ventricles ${ }^{6}$. This recommendation is largely based on numerous randomized control trials, demonstrating reduced all-cause mortality and/or hospitalization in CRT-treated patients ${ }^{7-10}$. However, $30 \%$ of patients show no response to $\mathrm{CRT}^{11,12}$, even some 
patients fulfilling strict criteria for $\mathrm{LBBB}^{13}$. The amount of response of a patient to CRT depends on the underlying pathology and the delivery of the pacing therapy ${ }^{14,15}$, delivery of the therapy can be modulated by choosing one or more good pacing sites and programming adequate delays of stimulation between the pacing electrodes ( $\mathrm{V} V$ and $\mathrm{V} V$ ).

\section{Optimizing pacing therapy}

In a cardiac pacemaker the moment of electrical stimulation and delay between different stimulations (pacing delay) can be programmed. Therefore the pacing delay is one of factors that can be used to optimize the benefit of pacing in a pacemaker recipient. Numerous studies have shown that atrio-ventricular delay can optimize ventricular filling while the ventriculoventricular delay can improve the ventricular activation pattern ${ }^{16-18}$. However, numerous optimization methods exist that provide different 'optimal' settings ${ }^{19}$. Furthermore, a metanalysis of pacing delay optimization studies in CRT showed no long-term benefits of optimizing pacing delay settings ${ }^{17}$.

In light of the abovementioned studies, it is not completely understood what parameter should be used to optimize pacemaker therapy. For example, most studies focus on LV function alone while right ventricular (RV) function is also affected by ventricular pacing ${ }^{20}$. Furthermore, several studies show that RV failure is an independent predictor of mortality in patients with LV failure with and without CRT ${ }^{21,22}$. These data indicate that a whole-heart approach to pacing therapy optimization should be adopted. In the present thesis, we will use computer simulations of cardiovascular system dynamics to perform such a whole-heart investigation of pacing therapy optimization. 


\section{Mechanical consequences of abnormal ventricular conduction}

As previously explained, electrical dyssynchrony leads to mechanical dyscoordination. Measuring mechanical dyscoordination can thus potentially be used for better selection of patients for CRT. Studies into CRT patient selection have mainly focused on better identifying patients with LBBB-like mechanical dyscoordinationn. In LBBB the septum is activated early compared to the LV lateral wall, resulting in early septal shortening and systolic septal rebound stretch. These abnormal deformations of the septum have been studied using echocardiography and were found to be associated with outcome in CRT patients ${ }^{23-25}$.

CRT response can potentially also be predicted using regional myocardial work, the integral of deformation and pressure over a cardiac cycle ${ }^{24,26}$. Recently, a method was introduced that allows measurement of this regional work through echocardiography combined with a simple brachial artery cuff pressure measurements ${ }^{27}$. In earlier animalexperimental studies invasive LV pressure and non-invasive measures of myocardial strain were used. These studies showed low, sometimes negative, myocardial work in the early-activated septum and enhanced myocardial work in the late-activated LV lateral wall during RV pacing ${ }^{28}$ and $\mathrm{LBBB}^{29}$. It was also previously shown that these measures of regional myocardial work relate to regional metabolism ${ }^{30}$ and changes in ventricular wall mass ${ }^{29}$.

The mechanical consequences of ventricular electrical dyssynchrony can be measured and appear to be associated to CRT response in numerous single center trials. Such measures have however not been added to the recent guidelines, indicating the additive value of these imaging based measures is debatable. It is still unknown how typical pathophysiological variations, such as hypertension, affect deformation and myocardial work. In the present thesis we will use computer simulations to assess if and how 
mechanical consequences of abnormal ventricular conduction are affected by more than ventricular conduction alone.

\section{Computational modeling of the paced heart}

Computer models provide a description of situations through simulations. The simulated outcome always depends on the relations and simulation conditions assumed. Computer simulations therethrough allow a level of control that is not always physically, ethically or financially possible within animal or clinical studies. Computer modeling thus enables forming of new insights, also in the context of cardiac pacing ${ }^{31-33}$. Many models were developed around the same time CRT was introduced. This led to computational studies on electrophysiology and mechanics at multiple levels (cell, tissue and organ scales) as well as hemodynamics (system scale) ${ }^{31}$. The ultimate goal of computer modeling in a cardiac pacing context is to maximize the efficacy and efficiency of pacing therapy in each patient.

In this thesis we used CircAdapt to study factors influencing cardiac pacing response. CircAdapt is a lumped parameter model that enables real-time beat-to-beat simulations of the mechanics and hemodynamics of the human cardiovascular system under physiological and pathophysiological circumstances ${ }^{34-36}$. The model consists of connected modules which represent the atria, ventricles, myocardial tissue, the pericardium, valves, large blood vessels, and a module representing the systemic and pulmonary vasculature. The CircAdapt model is particularly apt to simulate cardiac pacing because it describes the physiology and physics of atrioventricular coupling, the mechanical interaction between the ventricles as well as the interaction between the ventricles and the circulatory system that itself leads to ventricular hemodynamic interaction. The serial and parallel coupling of the LV and RV makes CircAdapt particularly useful for whole-heart simulations. 


\section{4 | Chapter 1}

CircAdapt has been used in the context of cardiac pacing to extend insights of existing clinical and animal experimental data ${ }^{37,38}$ or to form new hypotheses based on simulations alone ${ }^{39-41}$. This thesis builds further upon this work, using CircAdapt in a whole-heart approach to extend insights into the working mechanisms of cardiac pacing.

\section{General aim and outline of thesis}

In this thesis we wholeheartedly computationally assessed cardiac pacing to better understand how cardiac pacing therapy and patient selection thereof can be optimized. In line with this, the general aim of this thesis was to improve our mechanistic understanding of how the variable aspects of pacing therapy delivery influence cardiac pump function.

In Chapters $\mathbf{2}$ and $\mathbf{3}$ we investigated the functional consequences of optimization of pacing delays. Chapter $\mathbf{2}$ specifically focuses on how the hemodynamic response to optimization of the atrioventricular (AV) delay is affected by different timing factors. We used simulations in which interatrial delay, pacing site and heart rate were varied to check the effects on AV flow dynamics and global hemodynamics.

In Chapter $\mathbf{3}$ we extended analysis of previous canine experimental work from Strik et $\mathrm{al}^{16}$ with measurements of the RV. Measured electrical activation times were used as direct input for the timing differences in contraction of the RV and LV in the CircAdapt model. Differences between LV and RV hemodynamic responses were investigated and linked to the whole heart cardiac output that was available in the simulations.

Studies looking into the prediction of response to CRT often focus only on electrical measurements performed before CRT implant. In Chapter 4 we investigated whether pacing-induced electrical dyssynchrony at the time of cardiac resynchronization therapy (CRT) device implantation was 
associated with chronic CRT response. To assess pacing-induced electrical dyssynchrony we used electrograms, measured during CRT implant, to determine the interlead paced-to-sensed delays: the time interval between a pacing artefact at one electrode and the sensing artefact at the other electrode. The relative contributions of baseline dyssynchrony and interlead paced-to-sensed delays to CRT response were studied in a more well-controlled in silico environment using CircAdapt.

In Chapter 5 we investigated how changes in afterload affect measures of mechanical dyssynchrony and ventricular work in LBBB. The effect of afterload on selection and treatment of CRT patients is relevant since hypertension is a comorbidity, present in more than $60 \%$ of CRT and pacemaker patients ${ }^{42,43}$. In Chapter 6 we discussed and extended findings of a recent study by Storsten et $\mathrm{a}^{44}$, who for the first time looked into RV work in LBBB patient, showing that in patients with LBBB the RV free wall has a distinct early systolic shortening which coincides with the early systolic septal shortening. Creating LBBB in dogs also resulted in a decrease in both septal and RV free wall work, which increased again with CRT pacing. We investigated, using CircAdapt simulations, whether RV contractile failure would change this response and thereby also affect the effect of CRT as a whole.

To conclude, Chapter $\mathbf{7}$ integrates the major findings of all aforementioned chapters and puts them in a broader scientific and clinical perspective. 


\section{References}

1. Mulpuru SK, Madhavan M, McLeod CJ, Cha YM, Friedman PA. Cardiac Pacemakers: Function, Troubleshooting, and Management: Part 1 of a 2-Part Series. J Am Coll Cardiol. 2017;69(2):189-210. doi:10.1016/j.jacc.2016.10.061

2. Azadani PN, Soleimanirahbar A, Marcus GM, et al. Asymptomatic Left Bundle Branch Block Predicts New-Onset Congestive Heart Failure and Death From Cardiovascular Diseases. Cardio/Res. 2012;3(6):258-263. doi:10.4021/cr214w

3. Cheng A, Helm RH, Abraham TP. Pathophysiological mechanisms underlying ventricular dyssynchrony. Europace. 2009;11 Suppl 5:10-14. doi:10.1093/europace/eup272

4. $\quad$ Yu CM, Chan JYS, Zhang Q, et al. Biventricular pacing in patients with bradycardia and normal ejection fraction. N Engl J Med. 2009;361(22):2123-2134. doi:10.1056/NEJMoa0907555

5. Chen S, Wang Z, Kiuchi MG, et al. Cardiac pacing strategies and post-implantation risk of atrial fibrillation and heart failure events in sinus node dysfunction patients: a collaborative analysis of over 6000 patients. Clin Res Cardiol. 2016;105(8):687698. doi:10.1007/s00392-016-0973-1

6. Brignole M, Auricchio A, Baron-Esquivias G, et al. 2013 ESC Guidelines on cardiac pacing and cardiac resynchronization therapy: the Task Force on cardiac pacing and resynchronization therapy of the European Society of Cardiology (ESC). Developed in collaboration with the European Heart Rhythm Association . Eur Heart J. 2013;34(29):2281-2329. doi:10.1093/eurheartj/eht150

7. Moss AJ, Hall WJ, Cannom DS, et al. Cardiac-Resynchronization Therapy for the Prevention of Heart-Failure Events. N Engl J Med. 2009;361(14):1329-1338. doi:10.1056/NEJMoa0906431

8. Bristow MR, Saxon LA, Boehmer J, et al. Cardiac-Resynchronization Therapy with or without an Implantable Defibrillator in Advanced Chronic Heart Failure. N Engl J Med. 2004;350(21):2140-2150. doi:10.1056/NEJMoa032423

9. Cleland JGF, Daubert J-CC, Erdmann E, et al. The Effect of Cardiac Resynchronization on Morbidity and Mortality in Heart Failure. N Eng/ J Med. 2005;352(15):1539-1549. doi:10.1056/NEJMoa050496

10. Tang ASL, Wells GA, Talajic M, et al. Cardiac-Resynchronization Therapy for Mildto-Moderate Heart Failure. N Engl J Med. 2010;363(25):2385-2395. doi:10.1056/NEJMoa1009540

11. Naqvi SY, Jawaid A, Goldenberg I, Kutyifa V. Non-response to Cardiac Resynchronization Therapy. Curr Heart Fail Rep. 2018;15(5):315-321. doi:10.1007/s11897-018-0407-7

12. Zhang Q, Zhou Y, Yu C-M. Incidence, definition, diagnosis, and management of the cardiac resynchronization therapy nonresponder. Curr Opin Cardiol. 2015;30(1):40-49. doi:10.1097/HCO.0000000000000140 
13. Bertaglia E, Migliore F, Baritussio A, et al. Stricter criteria for left bundle branch block diagnosis do not improve response to CRT. Pacing Clin Electrophysiol. 2017;40(7):850-856. doi:10.1111/pace.13104

14. Mullens W, Grimm RA, Verga T, et al. Insights From a Cardiac Resynchronization Optimization Clinic as Part of a Heart Failure Disease Management Program. J Am Coll Cardiol. 2009;53(9):765-773. doi:10.1016/j.jacc.2008.11.024

15. Vernooy K, van Deursen CJM, Strik M, Prinzen FW. Strategies to improve cardiac resynchronization therapy. Nat Rev Cardiol. 2014;11(8):481-493. doi:10.1038/nrcardio.2014.67

16. Strik M, van Middendorp LB, Houthuizen $\mathrm{P}$, et al. Interplay of electrical wavefronts as determinant of the response to cardiac resynchronization therapy in dyssynchronous canine hearts. Circ Arrhythmia Electrophysiol. 2013;6(5):924-931. doi:10.1161/CIRCEP.113.000753

17. Auger D, Hoke U, Bax JJ, Boersma E, Delgado V. Effect of atrioventricular and ventriculoventricular delay optimization on clinical and echocardiographic outcomes of patients treated with cardiac resynchronization therapy: A metaanalysis. Am Heart J. 2013;166(1):20-29. doi:10.1016/j.ahj.2013.03.021

18. Bogaard MD, Meine M, Tuinenburg AE, Maskara B, Loh P, Doevendans PA. Cardiac resynchronization therapy beyond nominal settings: Who needs individual programming of the atrioventricular and interventricular delay? Europace. 2012;14(12):1746-1753. doi:10.1093/europace/eus170

19. Sohaib SMA, Whinnett ZI, Ellenbogen KA, et al. Cardiac resynchronisation therapy optimisation strategies: Systematic classification, detailed analysis, minimum standards and a roadmap for development and testing. Int J Cardiol. 2013;170(2):118-131. doi:10.1016/j.ijcard.2013.10.069

20. Quinn TA, Cabreriza SE, Richmond ME, Weinberg AD, Holmes JW, Spotnitz HM. Simultaneous variation of ventricular pacing site and timing with biventricular pacing in acute ventricular failure improves function by interventricular assist. $\mathrm{Am}$ $J$ Physiol Heart Circ Physiol. 2009;297(6):H2220-H2226. doi:10.1152/ajpheart.00802.2009

21. Groote PDE, Millaire A, Foucher-hossein C, et al. Right ventricular ejection fraction is an independent predictor of survival in patients with moderate heart failure. $J$ Am Coll Cardiol. 1998;32(4):948-954. doi:http://dx.doi.org/10.1016/S0735$1097 \% 2898 \% 2900337-4$

22. Ricci F, Mele D, Bianco F, Bucciarelli V, De Caterina R, Gallina S. Right heartpulmonary circulation unit and cardiac resynchronization therapy. Am Heart J. 2017;185:1-16. doi:10.1016/j.ahj.2016.11.005

23. De Boeck BWL, Teske AJ, Meine M, et al. Septal rebound stretch reflects the functional substrate to cardiac resynchronization therapy and predicts volumetric and neurohormonal response. Eur J Heart Fail. 2009;11(9):863-871. doi:10.1093/eurjhf/hfp107 
24. Vecera J, Penicka M, Eriksen $M$, et al. Wasted septal work in left ventricular dyssynchrony: A novel principle to predict response to cardiac resynchronization therapy. Eur Heart J Cardiovasc Imaging. 2016;17(6):624-632. doi:10.1093/ehjci/jew019

25. Gorcsan J, Anderson CP, Tayal B, et al. Systolic Stretch Characterizes the Electromechanical Substrate Responsive to Cardiac Resynchronization Therapy. JACC CardiovasC Imaging. 2019;12(9):1741-1752. doi:10.1016/j.jcmg.2018.07.013

26. Zweerink A, De Roest GJ, Wu L, et al. Prediction of Acute Response to Cardiac Resynchronization Therapy by Means of the Misbalance in Regional Left Ventricular Myocardial Work. J Card Fail. 2016;22(2):133-142. doi:10.1016/j.cardfail.2015.10.020

27. Russell K, Eriksen M, Aaberge $L$, et al. A novel clinical method for quantification of regional left ventricular pressurestrain loop area: A non-invasive index of myocardial work. Eur Heart J. 2012;33(6):724-733. doi:10.1093/eurheartj/ehs016

28. Prinzen FW, Hunter WC, Wyman BT, McVeigh ER. Mapping of regional myocardial strain and work during ventricular pacing: experimental study using magnetic resonance imaging tagging. J Am Coll Cardiol. 1999;33(6):1735-1742. doi:10.1016/S0735-1097(99)00068-6

29. Vernooy K, Verbeek XAAM, Peschar M, et al. Left bundle branch block induces ventricular remodelling and functional septal hypoperfusion. Eur Heart J. 2005;26(1):91-98. doi:10.1093/eurheartj/ehi008

30. Delhaas T, Arts T, Prinzen FW, Reneman RS. Regional fibre stress-fibre strain area as an estimate of regional blood flow and oxygen demand in the canine heart. $J$ Physiol. 1994;477(3):481-496. doi:10.1113/jphysiol.1994.sp020209

31. Lee AWC, Costa CM, Strocchi M, Rinaldi CA, Niederer SA. Computational Modeling for Cardiac Resynchronization Therapy. J Cardiovasc Trans/ Res. 2018;11(2):92-108. doi:10.1007/s12265-017-9779-4

32. Kerckhoffs RCP, Omens JH, Mcculloch AD. Mechanical discoordination increases continuously after the onset of left bundle branch block despite constant electrical dyssynchrony in a computational model of cardiac electromechanics and growth. 2012:65-72. doi:10.1093/europace/eus274

33. Russell K, Smiseth OA, Gjesdal $O$, et al. Mechanism of prolonged electromechanical delay in late activated myocardium during left bundle branch block. Am J Physiol - Hear Circ Physiol. 2011;301(6).

34. Arts T, Delhaas T, Bovendeerd P, Verbeek X, Prinzen F. Adaptation to mechanical load determines shape and properties of heart and circulation: the CircAdapt model. Am J Physiol Heart Circ Physiol. 2005;288:1943-1954. doi:10.1152/ajpheart.00444.2004.

35. Lumens J, Delhaas T, Kirn B, Arts T. Three-wall segment (TriSeg) model describing mechanics and hemodynamics of ventricular interaction. Ann Biomed Eng. 
2009;37(11):2234-2255. doi:10.1007/s10439-009-9774-2

36. Walmsley J, Arts T, Derval N, et al. Fast Simulation of Mechanical Heterogeneity in the Electrically Asynchronous Heart Using the MultiPatch Module. PLoS Comput Biol. 2015;11(7):e1004284. doi:10.1371/journal.pcbi.1004284

37. Leenders GE, Lumens J, Cramer MJ, et al. Septal Deformation Patterns Delineate Mechanical Dyssynchrony and Regional Differences in Contractility: Analysis of Patient Data Using a Computer Model. Circ Hear Fail. 2012;5(1):87-96. doi:10.1161/CIRCHEARTFAILURE.111.962704

38. Huntjens PR, Ploux S, Strik M, et al. Electrical Substrates Driving Response to Cardiac Resynchronization Therapy. Circ Arrhythmia Electrophysiol. 2018;11(4):e005647. doi:10.1161/CIRCEP.117.005647

39. Walmsley J, Huntjens PR, Prinzen FW, Delhaas T, Lumens J. Septal flash and septal rebound stretch have different underlying mechanisms. Am J Physiol Heart Circ Physiol. 2016;310(3):H394-403. doi:10.1152/ajpheart.00639.2015

40. Lumens J, Arts T, Broers B, et al. Right ventricular free wall pacing improves cardiac pump function in severe pulmonary arterial hypertension : a computer simulation analysis. 2009:2196-2205. doi:10.1152/ajpheart.00870.2009.

41. Huntjens PR, Walmsley J, Ploux S, et al. Influence of left ventricular lead position relative to scar location on response to cardiac resynchronization therapy: a model study. Europace. 2014;16 Suppl 4:iv62-iv68. doi:10.1093/europace/euu231

42. Massoullié G, Chouki C, Mulliez AA, et al. Effect of Optimization of Medical Treatment on Long-Term Survival of Patients With Heart Failure After Implantable Cardioverter Defibrillator and Cardiac Resynchronization Device Implantation (from the French National EGB Database). Am J Cardiol. 2018;121(6):725-730. doi:10.1016/j.amjcard.2017.12.013

43. Lee JH, Lee S-R, Choi E-K, et al. Temporal Trends of Cardiac Implantable Electronic Device Implantations: a Nationwide Population-based Study. Korean Circ J. 2019;49(10):1-12. doi:10.4070/kcj.2018.0444

44. Storsten P, Aalen JM, Boe E, et al. Mechanical Effects on Right Ventricular Function From Left Bundle Branch Block and Cardiac Resynchronization Therapy. JACC Cardiovasc Imaging. 2020;13(7). doi:10.1016/j.jcmg.2019.11.016 


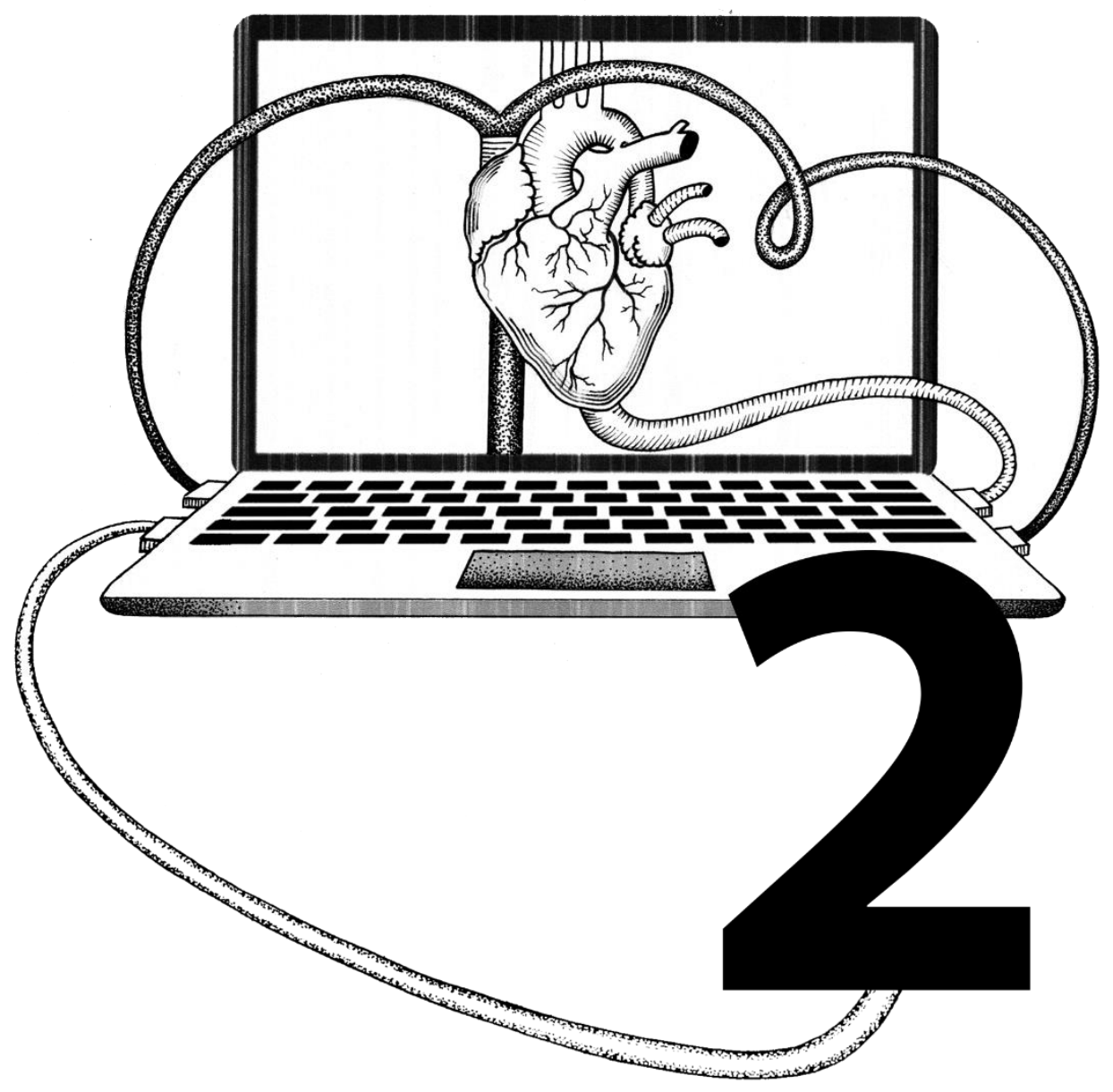




\section{Chapter 2}

Importance of effective atrioventricular delay for ventricular filling and cardiac pump function

Erik Willemen, Peter R. Huntjens, Tammo Delhaas, Joost Lumens, Frits W. Prinzen

In preparation 


\section{Abstract}

Adaptation of the atrio-ventricular delay $(A \vee d)$ during ventricular pacing affects ventricular filling and thereby cardiac pump function. In this study we use computer simulations to unravel the effect of heart rate, interatrial delay and ventricular pacing site on the optimal AVd. In addition, our aim was to develop a novel physiology-based strategy to determine the optimal $A V d$. The optimal $A V d$ was defined as the $A V d$ that provides the highest steady-state cardiac output (equal in in both systemic and pulmonary circulation). Effective $A V d(e A V d)$, defined as the interval between mean onset of atrial and mean onset of ventricular contraction, was therefore tested as a physiology-based alternative to define optimal timing differences between the atria and ventricles.

The simulations show that an increase in heart rate leads to a decrease in the optimal AVd. In contrast, the optimal AVd increases with increasing interatrial activation delay and with decreasing interventricular activation delay (such as during biventricular rather than right or left ventricular pacing). The latter observations suggest that both LV and RV filling dynamics determine the optimal AVd. Varying heart rate, inter-atrial delay and ventricular activation delay within physiological ranges resulted in a large range of optimal paced $A V d s$ (104 +/- $24 \mathrm{~ms})$. To correct for all these variation, whole-heart effective $\mathrm{AVd}(\mathrm{eAVd})$ is proposed. eAVd is defined as the interval between mean onset of atrial and mean onset of ventricular contraction, and was expressed in absolute terms and as \% of the cardiac cycle (\%eAVd). The optimal \%eAVd was $14.7+/-0.9 \%$, thus showing a considerably smaller variation than the absolute optimal paced $\mathrm{AVd}$. In conclusion, wholeheart effective $\mathrm{AVd}$ enables a more consistent definition of atrioventricular coupling, possibly improving and facilitating clinical optimization of the AVd. 


\section{Introduction}

During ventricular pacing, timing between atrial and ventricular contraction can be affected by setting the atrioventricular (AV) delay $(\mathrm{AVd})$. An optimal $\mathrm{AVd}$ promotes maximum contribution of atrial contraction to ventricular filling. If the $\mathrm{AVd}$ is too long, the atria contract too early in diastole. The passive mitral valve flow during the early filling phase therefore merges with the active transmitral flow resulting from the atrial contraction, characterized by a fusion of the E- and A-wave on echo-doppler. The large time delay between atrial and ventricular systole, as a result of a prolonged $\mathrm{AVd}$, also leaves time for diastolic regurgitation to occur. If the $A V d$ is too short, the ventricles contract too early, causing preliminary closure of the AV valve, limiting the contribution of the atrial contraction to ventricular filling.

Cardiac output can increase $9-21 \%$ in pacemaker patients with normal left ventricular (LV) function when going from a too short $A V d$ to a more physiological AVd (Manisty et al., 2012; Mehta et al., 1989; Wish et al., 1987). However, the optimal AVd varies between individuals and between pacemaker conditions. It is known that the optimal AVd is shorter when the atrial activation is sensed as compared to when the atrium is paced (Janosik et al., 1989; Wish et al., 1987). This may be explained by a larger inter-atrial activation delay (IAd) during atrial pacing, which shortens the actual delay between left atrial (LA) and LV contraction(Levin et al., 2011). Furthermore, differences in pacing site result in variations of the ventricular activation pattern and therefore the delay between the atrial and ventricular contraction. Therefore, we hypothesize that ventricular pacing site also affects the optimal AVd. Another factor that affects the optimal AVd is heart rate (Whinnett et al., $2006 \mathrm{~b})$, since increasing heart rate shortens the total diastolic time available for ventricular filling.

In the present study we aim to unravel the effect of heart rate, IAd and ventricular pacing site on the optimal AVd by performing simulations with a computer model of the heart and circulation. The mechanistic knowledge obtained from these simulations will be used to devise a novel physiology-based and universal strategy to determine the optimal AVd. 


\section{Methods}

\section{Computer simulations}

The CircAdapt model of the human heart and circulation (Walmsley et al., 2015) was used to characterize the effect of heart rate, IAd and pacing site on the cardiovascular mechanics and hemodynamics during variations in AVd. Previous experimental and clinical studies have shown that the CircAdapt model realistically relates local ventricular myofiber mechanics to global cardiovascular hemodynamics in the paced heart (Huntjens et al., 2014, 2018; Lumens et al., 2013, 2015; Walmsley et al., 2015,2016 ) The source code of the CircAdapt version used for this study can be freely downloaded from the CircAdapt website (http://www.circadapt.org).

\section{Simulation protocol}

In this study, we used a 2-stage approach: first, we investigated the individual effects of heart rate, IAd and ventricular pacing site on the relation between cardiac function, in terms of cardiac output and filling dynamics on the one hand, and paced AVd on the other hand; second, we used simulations with combinations of these factors to devise a more universal definition of optimal $\mathrm{AVd}$ that is less dependent on interindividual variations of heart rate, IAd and ventricular pacing site.

From a healthy heart reference simulation the three above mentioned factors were changed as follows. Heart rate was changed between 60 and $100 \mathrm{bpm}$, in steps of $10 \mathrm{bpm}$. IAd was changed through setting the difference in timing of mean onset of contraction between the right and left atrium. The IAd values tested in this study ranged from CircAdapt's default value of $28 \mathrm{~ms}$ to a maximum of $68 \mathrm{~ms}$, in steps of $10 \mathrm{~ms}$. LVonly, right ventricular (RV)-only and biventricular (BiV) pacing were simulated by implementing different ventricular activation patterns, as illustrated in Figure 1. In all our simulations, the paced AVd is defined as the time delay between first assumed electrical atrial activation and the first activated ventricular segment. Mean onset of right atrial contraction was assumed to be $40 \mathrm{~ms}$, based on a total right atrial electrical activation time of approximately $90 \mathrm{~ms}$ (Lemery et al., 2007). 
For each of the 75 unique combination of heart rate, IAd and ventricular pacing site, a hemodynamically steady-state baseline simulation was obtained. All other model parameters were the same for all of these baseline simulations, including a paced AVd of $120 \mathrm{~ms}$, cardiac output of $5.1 \mathrm{l} / \mathrm{min}$, and a mean arterial pressure of $90 \mathrm{mmHg}$. For each of the baseline simulations, cardiac output and mean arterial pressure were regulated by homeostatic control through changes of circulating blood volume and systemic vascular resistance. Finally, cardiac function and filling hemodynamics were simulated for paced $A V d s$ ranging between $50 \mathrm{~ms}$ and $250 \mathrm{~ms}$, in steps of $1 \mathrm{~ms}$. Each of these simulations was performed with homeostatic control turned off, until a new hemodynamic steady-state was reached.

Healthy human reference heart

\section{Simulated factors:}
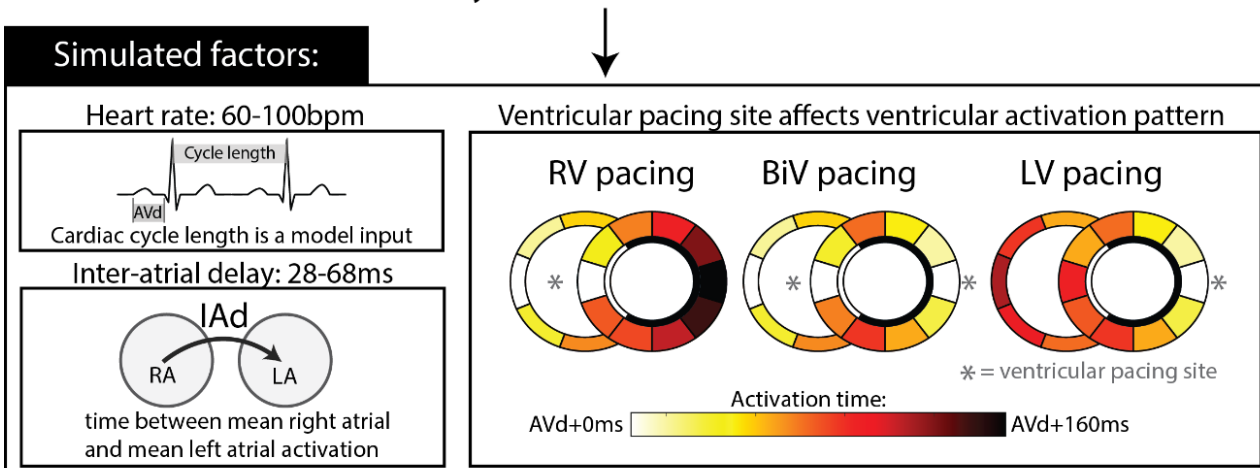

$$
\begin{gathered}
\text { baseline is adapted to a a systemic circulation with } \\
\text { a cardiac output of } 5.11 / \mathrm{min} \text { and mean arterial } \\
\text { pressure of } 90 \mathrm{mmHg} \text { at a AVd of } 120 \mathrm{~ms} \text {. }
\end{gathered}
$$

Unique baseline for each heart rate, IAd and pacing site combination

Figure 1 - Method of simulating the unique baseline conditions with changes in heart rate, inter-atrial delay (IAd) and ventricular pacing site. 


\section{Analysis of simulation results and the rationale behind the representation}

In this study, the effect of changes in $\mathrm{AVd}$ on pump function was quantified through cardiac output and LA-LV filling dynamics.

Considering that simulations run until a new hemodynamic steady-state is reached, any change in cardiac output reflects a change in global cardiac pump function.

Ventricular filling dynamics were studied in more detail by dividing the pattern of transmitral blood flow in passive, active and regurgitant flow. Active flow was defined as forward flow that occurs during active atrial contraction. Passive flow was defined as all forward flow occurring when the atrial myocardium was inactive. Regurgitant flow is defined as all retrograde flow across the $A V$ valve. In the model, the $A V$ valves only close when the distal pressure is equal to or larger than the proximal pressure and the tension of the distal myocardium is above a certain threshold value, the latter mimicking papillary muscle function. In other words, the AV valves allow late-diastolic regurgitant flow when the pressure gradient is negative and the distal myocardium is not activated.

\section{Analyzing left heart vs. whole-heart effective AVd}

In the second stage of the study we attempted to find a physiologybased strategy to determine the optimal AVd under different pacing conditions. To this end, we compared the paced $A V d$ with effective left heart and whole-heart effective AVd. Effective $A V d$ (eAVd) is defined as the interval between the mean onsets of atrial and ventricular contraction. In this study, these onset times of contraction are model input and can thus be used to calculate the eAVd. Since it has previously been hypothesized that the left heart AVd (i.e. LA to LV activation delay) is most important for $A V d$ optimization, we compared the left heart eAVd, based on the difference between mean LA-mean LV (free wall and septum) onset of contraction, to the whole-heart eAVd, based on the mean atrial ( $L A+R A)$ and mean ventricular ( $R V+L V)$ onset of activation. In addition, we performed the same analysis with eAVd normalized to the cardiac cycle time. 


\section{Results}

Figure 2 presents the contribution of active and passive filling as well as diastolic regurgitation to forward stroke volume at various $\mathrm{AVd}$ d at the mean of the simulated ranges. With increasing $\mathrm{AVd}$ there was an increase in active filling as well as regurgitation, while passive filling decreased. (middle panel). Forward stroke volume decreased at short $\mathrm{AVd}$ due to A-wave truncation (Figure 2, bottom left panel). Also, at long AVds forward stroke volume was suboptimal, mostly as a result of E-A wave fusion and the related reduction of passive filling and and due to diastolic regurgitation, both caused by the prolonged period between end of the A-wave and onset of ventricular contraction (Figure 2, bottom right panel). The net result of these changes was an optimum of net forward flow (and consequently stroke volume) at an AVd of $123 \mathrm{~ms}$ (Figure 2, top panel) for the simulated conditions (80 bpm, BiV pacing, IAd 48ms).
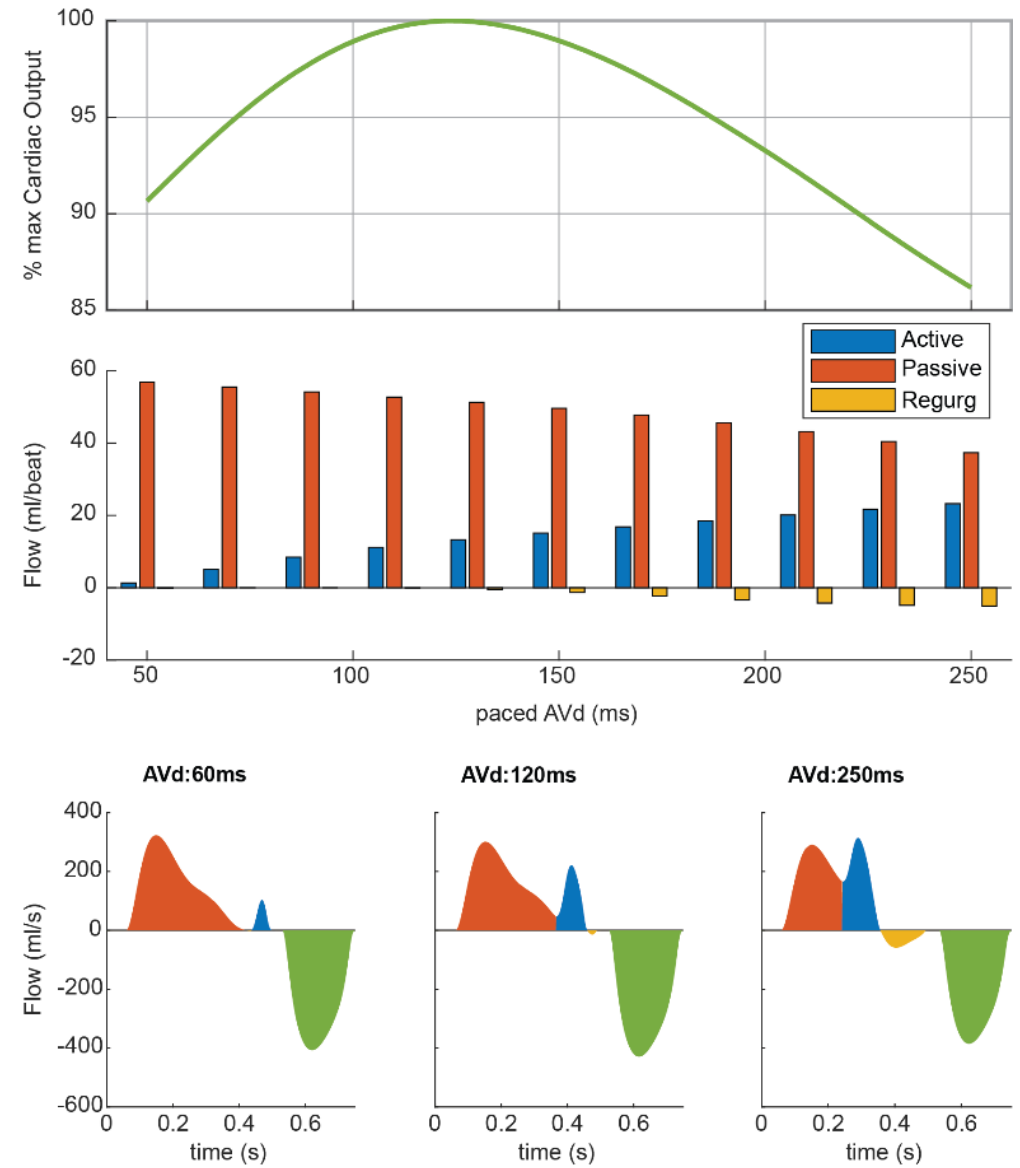

AVd:120ms

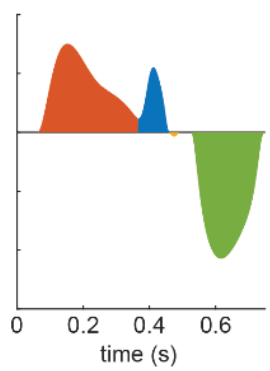

AVd:250ms

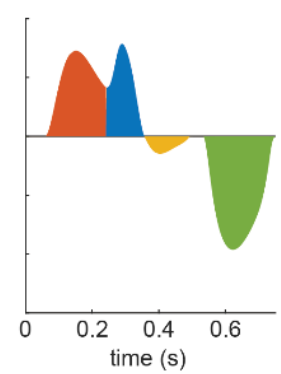

Figure 2 -

Cardiac output and flow distribution at different AVds at a heart rate of 80bpm, during BiV pacing and IAd of $48 \mathrm{~ms}$. 


\section{Effect of heart rate on the optimal AVd}

Figure 3 shows the dependence of cardiac output on AVd at three different heart rates. The optimal $\mathrm{AVd}$ decreased with increasing heart rate, being $141 \mathrm{~ms}$ at $60 \mathrm{bpm}, 123 \mathrm{~ms}$ at $80 \mathrm{bpm}$, and $95 \mathrm{~ms}$ at $100 \mathrm{bpm}$. The effect of $A V d$ and heart rate variations on mitral valve flow are shown in Figure 4.

At a heart rate of $60 \mathrm{bpm}$, passive flow was rather constant across the entire range of $\mathrm{AVd}$ (Figure 4, left), with the duration of the diastolic phase being sufficient to prevent E-A wave fusion. When increasing AVd from $50 \mathrm{~ms}$ to the optimal value of $141 \mathrm{~ms}$, active mitral flow increased by the reduction of A-wave truncation. Consequently cardiac output was increased by $10 \%$ (Figure 3 ). When the $\mathrm{AVd}$ was prolonged beyond the optimal AVd, active flow no longer changed whereas end-diastolic regurgitant flow gradually increased, leading to a decrease of cardiac output.

At a heart rate of $100 \mathrm{bpm}$, cardiac cycle length is shorter leading to a reduction in diastole duration. As a consequence, every increase in active flow led to a decrease in passive flow by E-A wave merging (Figure 4, right). By increasing AVd from 50ms to the optimal AVd of 95, the gain in active flow was larger than the loss in passive flow. When $A V d$ was prolonged beyond the optimal $A V d$, the combination of increased regurgitant flow and decreased passive flow surpassed the gain in active flow resulting in a decrease in cardiac output of up to $11 \%$ (Figure 3).

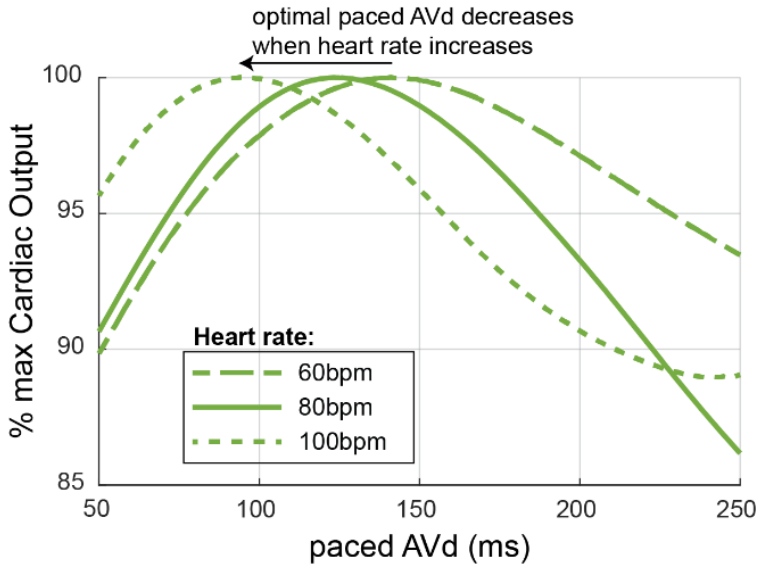

Figure 3 - Change in cardiac output with a change in $\mathrm{AV}$ delay at 60, 80 and $100 \mathrm{bpm}$. 


\section{Effect of inter-atrial delay on the optimal AVd}

Figure 5 demonstrates the effect of IAd on the optimal AVd. The optimal $A V d$ was increased when the IAd was prolonged. However, the increase in optimal AVd was always smaller than the change in IAd. An increase of $40 \mathrm{~ms}$ in IAd (28ms to $68 \mathrm{~ms}$ ) shifted the optimal paced AVd with $22 \mathrm{~ms}$, from $114 \mathrm{~ms}$ to $136 \mathrm{~ms}$.

\section{Effect of ventricular pacing site on AV dynamics}

Figure 6 shows the effect of pacing site on AV dynamics. The optimal AVd's during LV and RV pacing were 101 and 96 ms respectively, i.e. 2227 ms less than the optimal AVd during BiV pacing. However, the contribution of the three components of ventricular filling showed considerable differences between RV and LV pacing. Due to the late activation of the LV free wall, diastolic regurgitation was more pronounced during RV pacing.

The simulations revealed that BiV pacing leads to an optimal cardiac output at a longer AVd due to a larger degree of A-wave truncation at short AVds, as compared to RV and LV pacing (figure 7). Importantly, the similar shift in optimal AVd during RV and LV pacing is accomplished by very different contributions of passive and active filling, the latter being considerably larger during RV pacing, along with a larger regurgitation. 

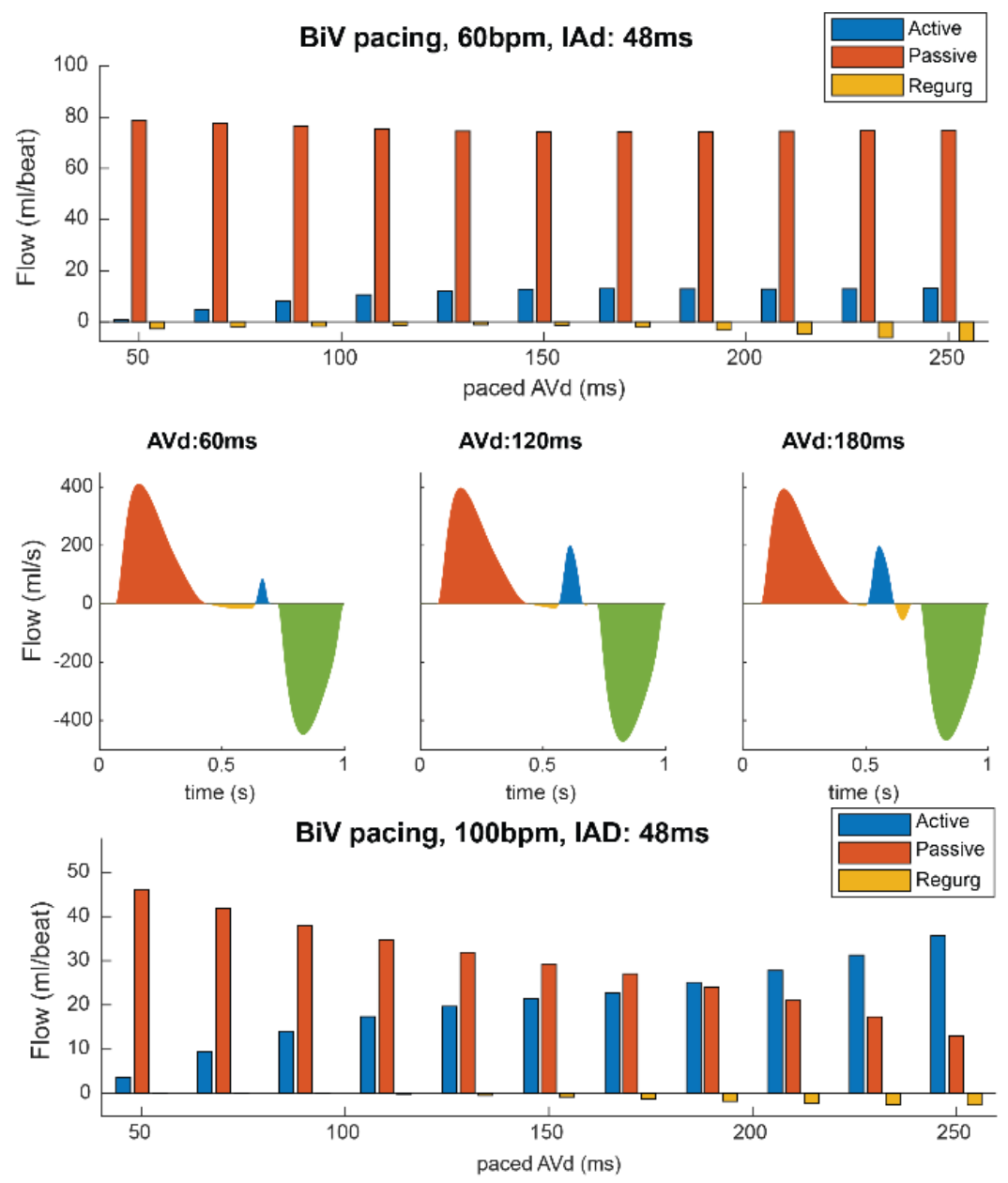

AVd:60ms

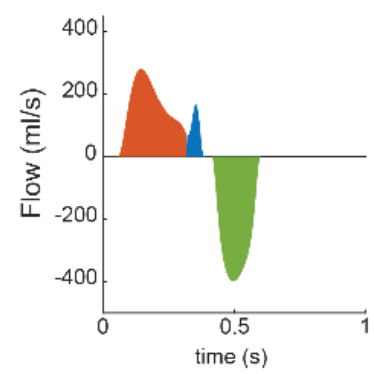

AVd:120ms

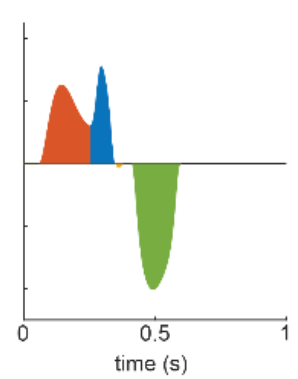

AVd: $180 \mathrm{~ms}$

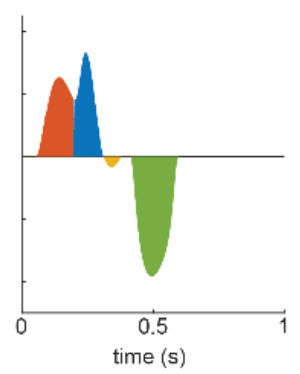

Figure 4 - Flow distributions at different AVds for a heart rate of $60 \mathrm{bpm}$ (left) and 100bpm (right). 


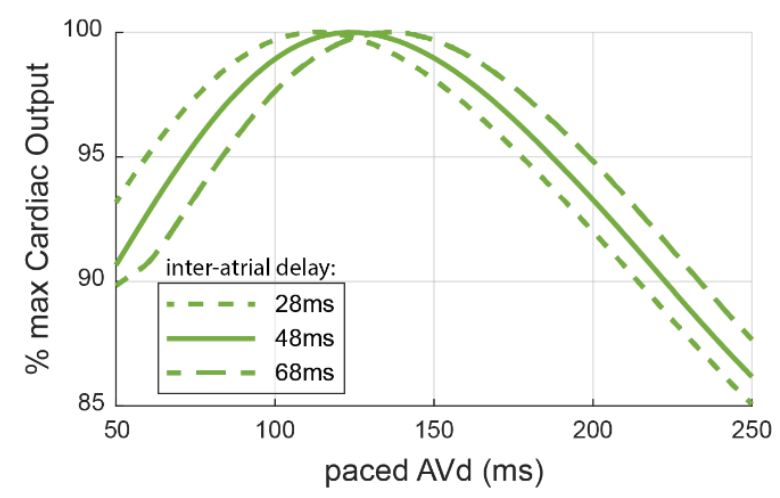

Figure 5 - Cardiac output as percentage of the maximum at different AVds for different inter-atrial delays (IAd) at a heart rate of $80 \mathrm{bpm}$ and BiV pacing.
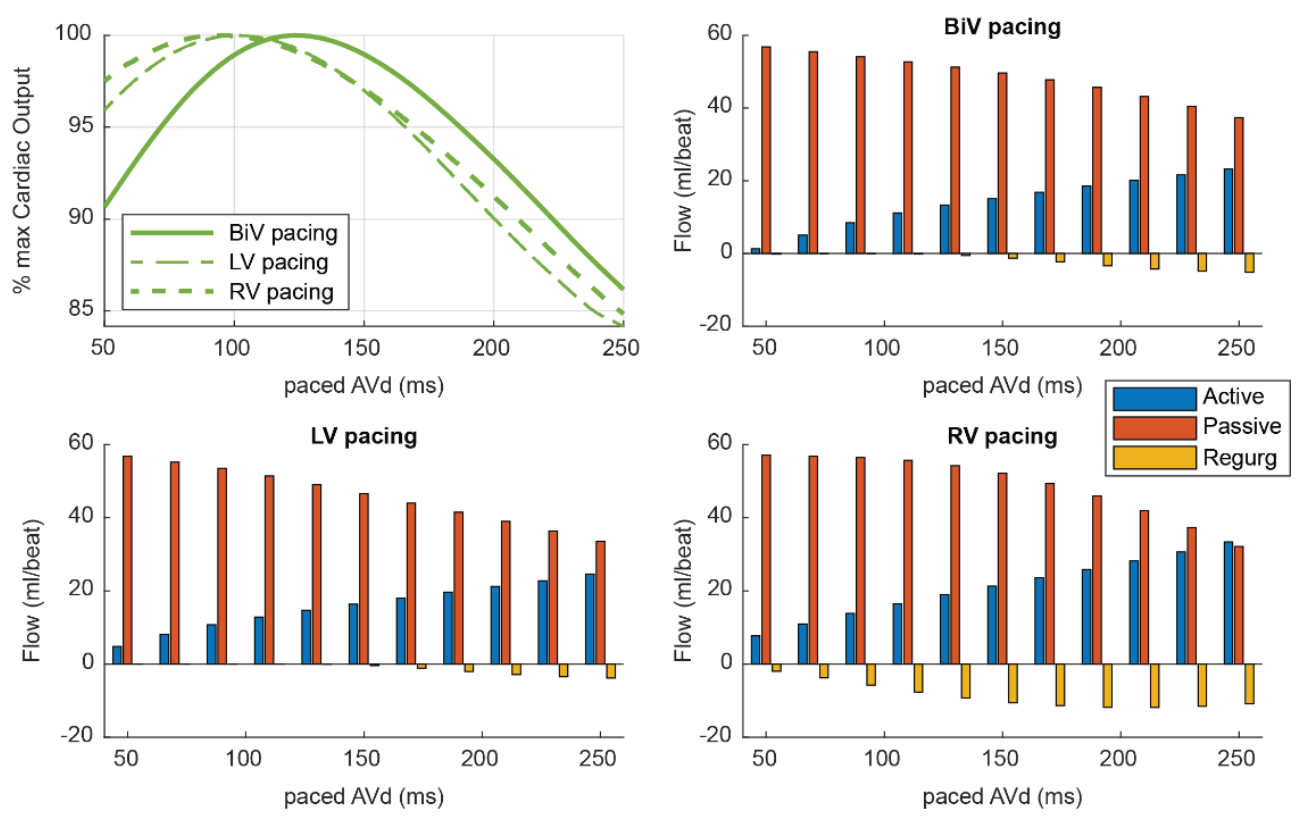

Figure 6 - Cardiac output and flow distribution at different AVds for BiV, LV and $R V$ pacing 

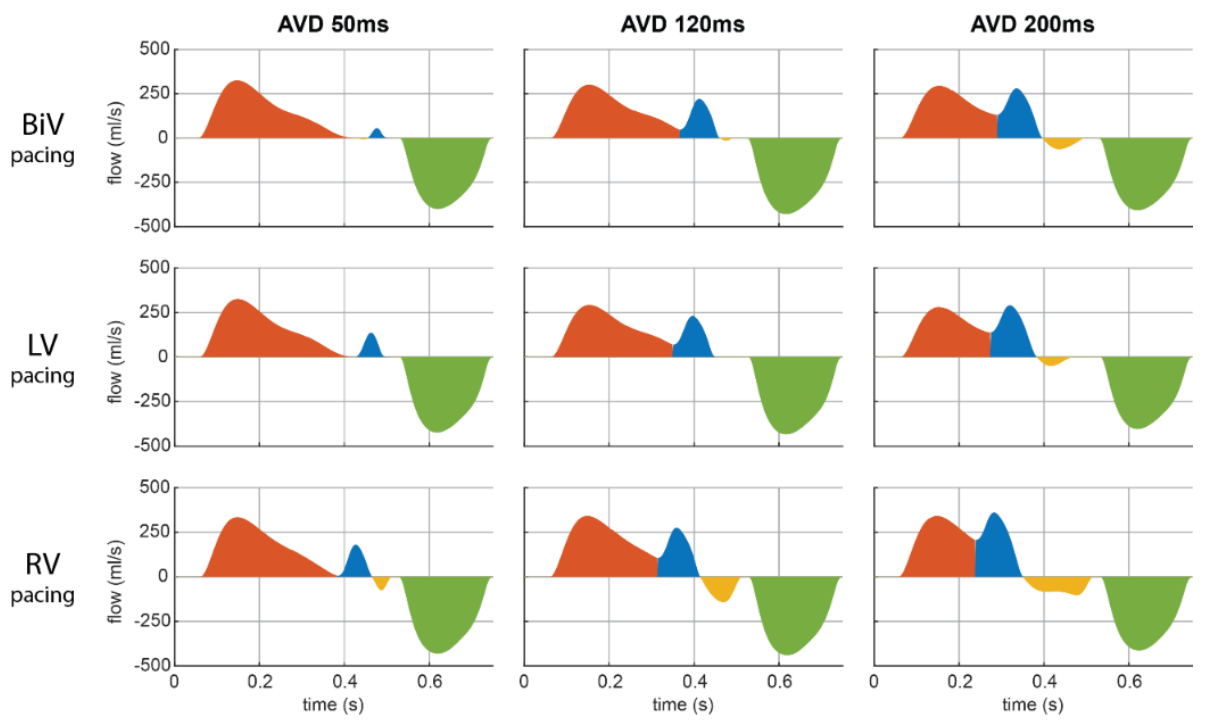

Figure 7 - Mitral valve flow at 50,120 and 200ms in the BiV, LV and RV pacing simulations at a heart rate of $80 \mathrm{bpm}$ and IAd of $48 \mathrm{~ms}$.

\section{Left heart vs. whole-heart effective AVd}

Figure 8 shows all cardiac output results of the simulations performed for this study for six different $A \bigvee d$ definitions. For all calculation methods, AVd relative to cycle length (Figure 8, right side) had less variance in the optimal $\mathrm{AVd}$ compared to $\mathrm{AVd}$ as an absolute time interval (Figure 8 , left side). The least variance in and smallest range of the optimal $A V d$ value was observed for relative whole-heart eAVd.

The range and variance of optimal $A V d$ only decreased slightly when comparing left heart eAVd to paced AVd, for both absolute (Figure $8 \mathrm{~A}$ vs. C) and relative (Figure $8, B$ vs. D) delays. Absolute optimal wholeheart eAVd had a smaller range and variance compared to optimal left heart eAVd (Figure 8, C vs. E). In Figure 8E, whole-heart eAVd curves with different IAds and pacing sites but with the same heart rate (color) form clusters. By taking the AVd relative to the cycle length, the smallest range in cardiac output at the same relative delay was observed for relative whole-heart eAVd (Figure $8 \mathrm{~F}$ ). At the mean of all optimal relative whole-heart eAVds, i.e. at $14.7 \%$ of the cycle time, the maximum decrease in cardiac output was only $0.4 \%$. At the mean of all optimal 
relative left heart eAVds (i.e. at $13.8 \%$ ), cardiac output decreased up to $0.9 \%$. For all other representations in Figure 8 , the largest decrease of cardiac output at the mean of all optimal AVds was $>2 \%$.
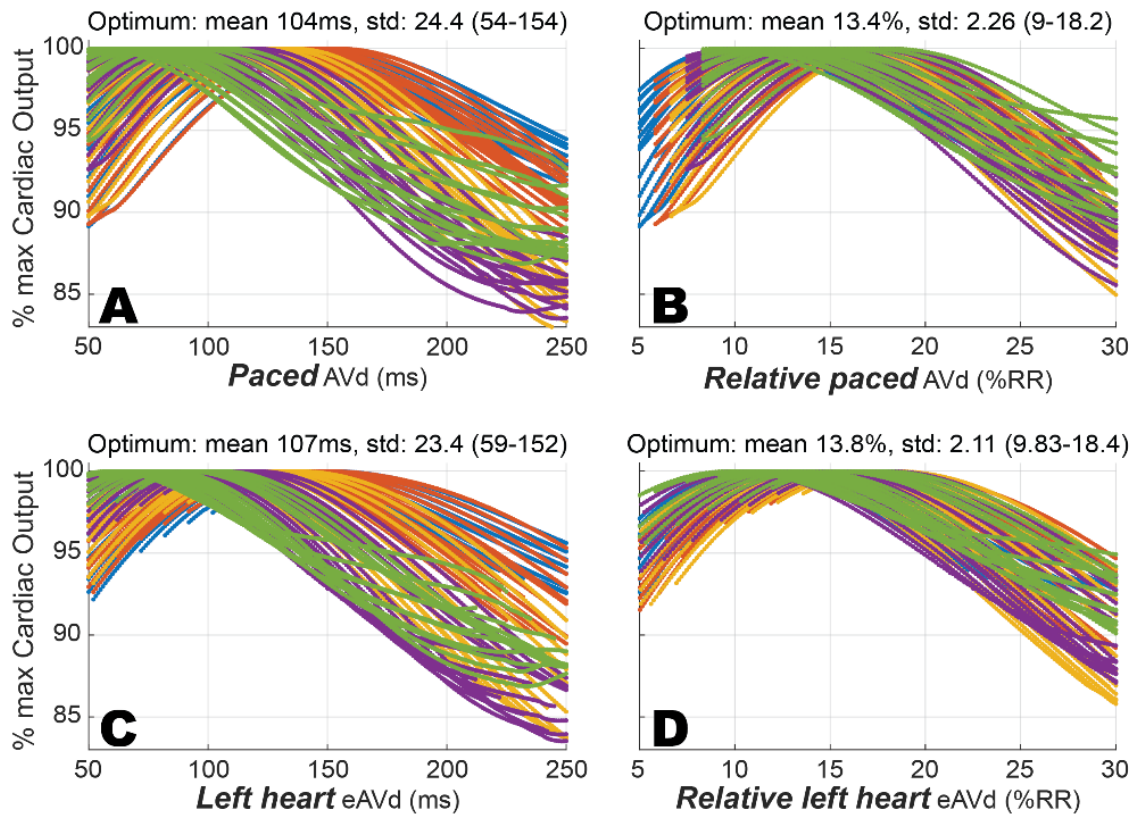

Optimum: mean 13.8\%, std: 2.11 (9.83-18.4)
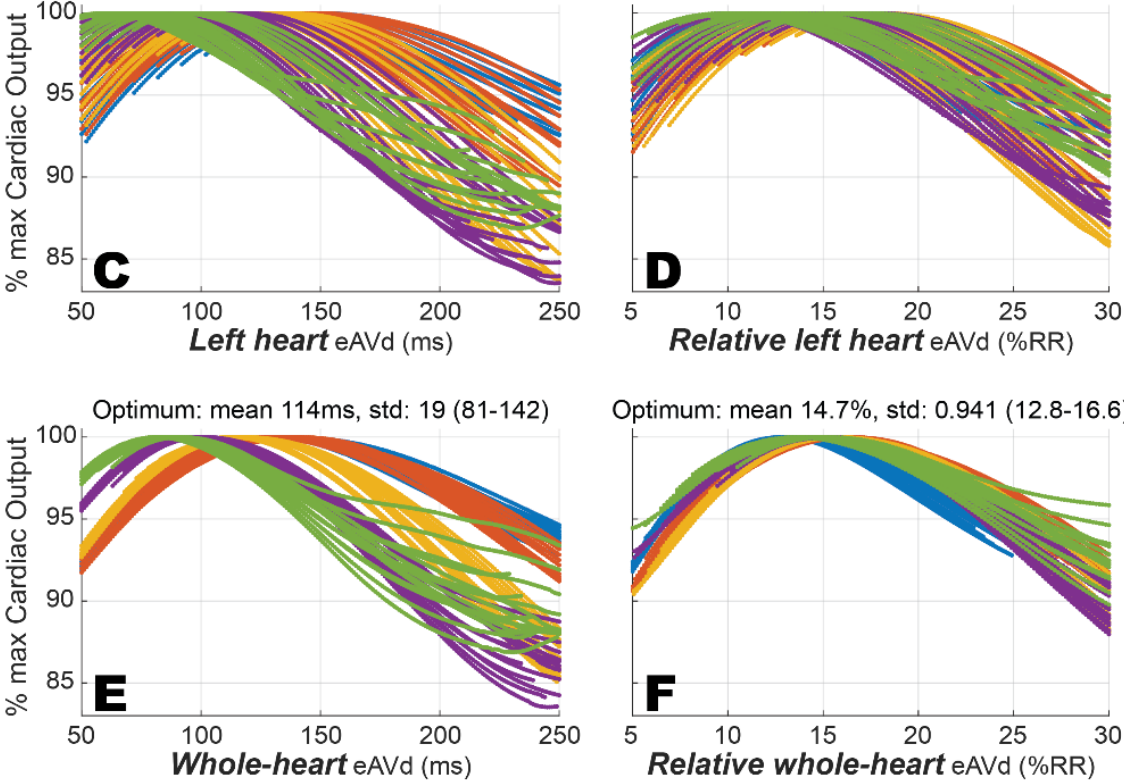

Heart rate: $60 \mathrm{bpm} 70 \mathrm{bpm} \quad 80 \mathrm{bpm}$ 90bpm $100 \mathrm{bpm}$

Figure 8 - Change in cardiac output with change in AVd at different heart rates, $I A D s$ and pacing sites ( $L V p, B i V p, R V p)$. The same simulation results are presented for six different axes with AVd in absolute values (ms, left) and relative to the cycle length (\%RR, right). Paced $A V d s$ are presented in the top $(A \& B)$, left heart eAVds in the middle (C\&D) and whole-heart eAVds in the bottom (E\&F). Each color indicates a different heart rate. 


\section{Discussion}

The main finding of this study is that variations in inter-atrial delay, ventricular pacing site and heart rate change the optimal paced AVd that results in maximal cardiac output. Consequently, it remains challenging to determine the pacing setting that leads to optimal hemodynamics based on the paced AVd. By using the mechanistic knowledge obtained from the computer simulations performed in the first part of the study we were able to devise a more robust definition of optimal AV pacing settings. Relative whole-heart effective AVd normalized to the cardiac cycle length appears to be a definition of the optimal $A V d$ that is relatively independent of heart rate, inter-atrial activation delay or ventricular pacing site.

\section{Heart rate modulates the optimal AVd}

The results of this study show that an increase in heart rate results in a decrease in optimal paced AVd. Multiple other studies have demonstrated the same shortening in optimal $\mathrm{AVd}$ with increase in heart rate (Kyriacou et al., 2018; Rafie et al., 2012; Whinnett et al., 2006a). Melzer et al. only found a significant decrease in optimal AVd during atrial pacing (DDD-mode) and not during atrial sensing (VDD-mode) (Melzer et al., 2008). This could possibly be explained by the smaller absolute change during sensing, due to a lower overall optimal paced $\mathrm{AVd}$, making it harder to reach significance.

The simulations in this study were performed on a healthy reference heart with a preserved ejection fraction. It has been observed that the rate-dependent decrease in optimal $\mathrm{AVd}$ was smaller in patients than in healthy controls (Sun et al., 2012). Scharf et al. even found an increase in optimal AVd with increasing heart rate during exercise in patients with systolic heart failure (Scharf et al., 2005). A possible reason for the difference could be changes in the relation between heart rate and the interval of diastole/systole in failing vs. healthy hearts. 


\section{Whole-heart effective AV delay: a guide to the 'best' compromise}

The results of this study are in line with the hypothesis that variations in the inter-atrial conduction time and pacing site change the optimal paced AVd. Most importantly, the results emphasize that function of both RV and LV should be considered, because pulmonary and systemic circulation are coupled in series. For each ventricle there is an optimal $\mathrm{AVd}$. When the ventricular optimum differs between ventricles initially, a balance is reached under hemodynamic steady-state conditions, resulting in one optimal AVd. For a given paced AVd, actual optimal RARV and LA-LV delay may differ with differences in inter atrial delay and ventricular activation delay, thereby changing the optimal (steady-state) paced AVd.

For LV function, the effect of IAd is known from studies focusing on the difference between atrial-sense and atrial-pace pacing, showing that due to longer IAd during atrial-pace pacing (Levin et al., 2011), the paced AVd should be longer (Kyriacou et al., 2018; Liang et al., 2011). Similar to our simulation results, the increase in optimal AVd was less than the increase in inter-atrial conduction time in the study by Levin et al (slope $<1$ ). This suggests that the LA-LV interval, which changes 1-on1 (slope=1) with IAd, is not the only factor that determines the optimal paced AVd. Our study, for the first time, expands on this by demonstrating that changes in interventricular delay can similarly affect the optimal paced AVd.

Almost all research into AV optimization focus on the left side of the heart. While these previously published studies support our findings based on measurements in the LV, there are no studies focusing on RV function or the circulation as a whole. Although the flow patterns presented in this study were from the left heart, we also studied the whole-heart response to AV optimization through whole-heart steadystate cardiac output. The focus on LV response could lead to the assumption that effective LA-LV contraction timing is the only factor that should be accounted for when choosing the optimum paced AVd. The results for the IAd already highlighted that the LA-LV delay is not the only factor affecting the whole-heart steady-state cardiac output, thereby indicating a role for the right atrium and ventricle. This becomes even more clear when comparing RV and LV pacing simulations. The mean LV activation was $37 \mathrm{~ms}$ later during RV compared to LV pacing in 
Figure 6, where the optimal AVd only differed by $7 \mathrm{~ms}$ between these pacing configurations. The RV free wall is however activated $56 \mathrm{~ms}$ later during LV compared to RV pacing. When the mean activation time of all ventricular segments is compared the difference in ventricular activation between RV and LV pacing is $6 \mathrm{~ms}$, i.e. very close to the $7 \mathrm{~ms}$ shift in optimal AVd. This suggested that it is essential to take into account the activation times of both atria and both ventricles when choosing the optimal AVd.

The results of the present study demonstrate that relative whole-heart effective atrio-ventricular contraction interval, also including the right atrial and right ventricular contraction timing, provide a better indication of when atrio-ventricular interaction leads to the highest cardiac output. The largest differences between relative left and whole-heart eAVd exist during RV pacing, where the LV free wall is activated late compared to the RV free wall. The observation that left heart eAVd provides less overlap between the different simulation conditions than whole-heart eAVd, especially for this RV paced simulations, indicates that the right heart also significantly contributes to the steady-state cardiac output and highlights the importance of a whole-heart assessment.

\section{Lack of a whole-heart approach may explain inefficacy of existing AV optimization strategies}

AV filling patterns were not the same at each relative whole-heart eAVd, neither is it the same at each optimal AVd. Interestingly, even though IAd and pacing site significantly change the flow distribution, the time between mean atrial and mean ventricular onset of contraction relative to the beat duration provides a consistent answer for the optimum. This suggests that flow distribution itself is not a determinant of the optimum even though it is one of the techniques typically used in AVd optimization. While different strategies using mitral flow velocity exist, the main goal of these methods is to prevent A-wave truncation and E-A fusion(Sohaib et al., 2013). While preventing truncation and fusion will prevent selecting the worst settings it does not always enable selecting the optimal $\mathrm{AVd}$. This becomes most clear in case a patient presents with a high heart rate. During a high heart rate E-A fusion always occurs when there is no A-wave truncation. Therefore, a balance between fusion and truncation is sometimes required. Other methods use the 
time of the end of the A-wave (at a long known $A V d$ ) in combination with the time of onset of mitral regurgitation(Jones et al., 2014). Such a method allows indirect determination of actual LA activation (through the A-wave end) and LV activation delay (through regurgitation) but does not completely incorporate the whole-heart activation. The observation that mitral valve flow(velocity) is not able to capture wholeheart effective AVd could be one of the reasons no long-term benefits of $\mathrm{AVd}$ optimization where found in the past using these methods and why it is not implemented within the current guidelines. Besides flow distribution other strategies use the electrograms of pacing electrodes (Ellenbogen et al., 2010; Singh et al., 2013). However, it is unclear how these relate to whole-heart eAVd since details about these algorithms are not available. As discussed below, pacemaker electrodes can potentially be implemented to determine whole-heart eAVd.

\section{Clinical implications and future perspective}

Our simulations indicate that differences in IAd, pacing site(s) and heart rate within and between patients can change the optimal paced $A V d$. In the default range of paced $A V d s$, between 100 and $150 \mathrm{~ms}$, this can lead to a change in cardiac output by up to $8 \%$. Results of this study also show that the pattern of transmitral blood flow at the optimum setting is not the same for all simulated conditions. Therefore, optimization based on the LV filling pattern alone is unlikely to provide the true optimal $\mathrm{AVd}$ under all conditions.

The whole-heart eAVd, which can be seen as a global, bilateral measure of AV coupling, provides a physiologically optimal AVd. In order to implement whole-heart eAVd in clinical practice, detailed information about right and left atrial and right and left ventricular activation is required. Future research may investigate whether relatively simple measurements like P-wave duration, inter-lead conduction times and/or interventricular mechanical dyssynchrony (speckle tracking or echoDoppler derived differences of aortic and pulmonary valve opening times) can be used to calculate whole-heart effective AVd. Ideally, the required measures should be implemented within a pacemaker, since that would enable continuous automatic optimization. Commercial optimization algorithms incorporating the electrograms derived from the pacing electrode exist. However, this approach did not show improvement over conventional echocardiographic optimization in large clinical trials(Ellenbogen et al., 2010; St. Jude Medical, 2019). The 
exact methodology of these algorithms is proprietary and therefore unknown. We think that a first step in creating a better algorithm would be to determine if pacing lead electrograms and/or echocardiographic measures can be used to reliably estimate effective whole-heart AVd. Such a study would require a 'gold-standard' for determining the effective whole-heart AVd such as an animal experimental or ex vivo setup including a large amount of electrodes on both the atria and ventricles. If a reliable estimate for effective whole-heart $A V d$ is found, a second study can investigate how this estimate should be normalized to heart rate in different patient populations. Based on these studies a third, very essential, study can investigate whether estimated effective whole-heart $A V d$, relative to heart rate, truly performs better than a fixed $\mathrm{AVd}$, in terms of long-term clinical outcome after pacemaker implant.

\section{Limitations}

The simulation results obtained in this study should be extrapolated to the real-world clinical situation with care, because the model uses a number of assumptions that may not be valid in patients. First of all, normal contractility of the ventricular and atrial myocardium was assumed. Also, we assumed the condition of complete AV block, whereas in many patients there may be AV conduction. In such situation, fusion of the pacing-induced activation wave(s) and intrinsic conduction may occur, thereby changing activation sequence. We chose to simulate complete AVB, because this allowed observing the isolated effect of changes in AV dynamics alone. Nevertheless, we hypothesize that the physiological principle of whole-heart effective AVd being the key component for ventricular filling also holds in case of fusion.

Simulations were performed assuming no homeostatic regulation of arterial pressure or systemic flow. In the in vivo situation, however, an acute change of $\mathrm{AVd}$ is likely to provoke an autonomic regulatory response, characterized by changes in peripheral arterial resistance and pressure, but not necessarily changes in the cardiac output response (Manisty et al., 2012). Notwithstanding, while the quantitative results might differ the overall effects observed in these simulations are likely to remain even though some aspects of the model could be improved in the future. In addition, the model provides the ideal platform to further investigate the importance of such inter-individual differences for cardiac function during pacing delay optimization. 


\section{Conclusion}

Simulations of whole-heart mechanics and circulatory hemodynamics during $\mathrm{AVd}$ optimization revealed that maximum cardiac output is achieved at various paced AVd settings, depending on heart rate, interatrial activation delay, and ventricular pacing site(s). Optimal paced AVd decreased with increasing heart rate and inter-ventricular delay, while it increased with right-to-left inter-atrial activation delays. Whole-heart effective AVd normalized to the cardiac cycle length consistently revealed cardiac output to be maximal if whole-heart eAVd was between $14-15 \%$ of the total cycle time. Whole-heart effective AVd enables a more consistent definition of how the atria and ventricles interact optimally which could potentially be applied for clinical AVd optimization. 


\section{References}

Ellenbogen, K. A., Gold, M. R., Meyer, T. E., Fernández Lozano, I., Mittal, S., Waggoner, A. D., et al. (2010). Primary results from the smartdelay determined AV optimization: A comparison to other AV delay methods used in cardiac resynchronization therapy (SMART-AV) trial: A randomized trial comparing empirical, echocardiographyguided, and algorithmic atrioventr. Circulation 122, 2660-2668. doi:10.1161/CIRCULATIONAHA.110.992552.

Huntjens, P. R., Ploux, S., Strik, M., Walmsley, J., Ritter, P., Haissaguerre, M., et al. (2018). Electrical Substrates Driving Response to Cardiac Resynchronization Therapy. Circ. Arrhythmia Electrophysiol. 11, e005647. doi:10.1161/CIRCEP.117.005647.

Huntjens, P. R., Walmsley, J., Ploux, S., Bordachar, P., Prinzen, F. W., Delhaas, T., et al. (2014). Influence of left ventricular lead position relative to scar location on response to cardiac resynchronization therapy: a model study. Europace 16 Suppl 4, iv62-iv68. doi:10.1093/europace/euu231.

Janosik, D. L., Pearson, A. C., Buckingham, T. A., Labovitz, A. J., Redd, R. M., and Mrosek, D. (1989). The hemodynamic benefit of differential atrioventricular delay intervals for sensed and paced atrial events during physiologic pacing. J. Am. Coll. Cardiol. 14, 499-507. doi:10.1016/0735-1097(89)90208-8.

Jones, S., Shun-Shin, M. J., Cole, G. D., Sau, A., March, K., Williams, S., et al. (2014). Applicability of the iterative technique for cardiac resynchronization therapy optimization: full-disclosure, 50-sequential-patient dataset of transmitral Doppler traces, with implications for future research design and guidelines. Eur. Eur. pacing, arrhythmias, Card. Electrophysiol. J. Work. groups Card. pacing, arrhythmias, Card. Cell. Electrophysiol. Eur. Soc. Cardiol. 16, 541-550. doi:10.1093/europace/eut257.

Kyriacou, A., Rajkumar, C. A., Pabari, P. A., Sohaib, S. M. A., Willson, K., Peters, N. S., et al. (2018). Distinct impacts of heart rate and right atrial-pacing on left atrial mechanical activation and optimal AV delay in CRT. PACE - Pacing Clin. Electrophysiol. 41, 959966. doi:10.1111/pace.13401.

Lemery, R., Birnie, D., Tang, A. S. L., Green, M., Gollob, M., Hendry, M., et al. (2007). Normal atrial activation and voltage during sinus rhythm in the human heart: An endocardial and epicardial mapping study in patients with a history of atrial fibrillation. J. Cardiovasc. Electrophysiol. 18, 402-408. doi:10.1111/j.15408167.2007.00762.x. 
Levin, V., Razavi, M., Coll, R., Coles, J. A. J., and Sambelashvili, A. (2011). Interatrial conduction correlates with optimal atrioventricular timing in cardiac resynchronization therapy devices. Pacing Clin. Electrophysiol. 34, 443-449. doi:10.1111/j.1540-8159.2010.02988.x.

Liang, H. Y., Cheng, A., Chang, K. C., Berger, R. D., Agarwal, K., Eulitt, P., et al. (2011). Influence of atrial function and mechanical synchrony on LV hemodynamic status in heart failure patients on resynchronization therapy. JACC Cardiovasc. Imaging 4, 691-698. doi:10.1016/j.jcmg.2011.02.019.

Lumens, J., Ploux, S., Strik, M., Gorcsan, J., Cochet, H., Derval, N., et al. (2013). Comparative electromechanical and hemodynamic effects of left ventricular and biventricular pacing in dyssynchronous heart failure: Electrical resynchronization versus left-right ventricular interaction. J. Am. Coll. Cardiol. 62, 2395-2403. doi:10.1016/j.jacc.2013.08.715.

Lumens, J., Tayal, B., Walmsley, J., Delgado-Montero, A., Huntjens, P. R., Schwartzman, D., et al. (2015). Differentiating Electromechanical from Non-Electrical Substrates of Mechanical Discoordination to Identify Responders to Cardiac Resynchronization Therapy. Circ. Cardiovasc. Imaging 8, 1-12. doi:10.1161/CIRCIMAGING.115.003744.

Manisty, C. H., Al-Hussaini, A., Unsworth, B., Baruah, R., Pabari, P. A., Mayet, J., et al. (2012). The acute effects of changes to AV delay on BP and stroke volume: potential implications for design of pacemaker optimization protocols. Circ. Arrhythm. Electrophysiol. 5, 122-130. doi:10.1161/CIRCEP.111.964205.

Mehta, D., Gilmour, S., Ward, D. E., and Camm, A. J. (1989). Optimal atrioventricular delay at rest and during exercise in patients with dual chamber pacemakers: a noninvasive assessment by continuous wave Doppler. Heart 61, 161-166. doi:10.1136/hrt.61.2.161.

Melzer, C., Bondke, H., Körber, T., Nienaber, C. A., Baumann, G., and Ismer, B. (2008). Should we use the rate-adaptive AV delay in cardiac resynchronization therapypacing? Europace 10, 53-58. doi:10.1093/europace/eum257.

Rafie, R., Qamruddin, S., Ozhand, A., Taha, N., and Naqvi, T. Z. (2012). Shortening of atrioventricular delay at increased atrial paced heart rates improves diastolic filling and functional class in patients with biventricular pacing. Cardiovasc. U/trasound 10, 2. doi:10.1186/1476-7120-10-2.

Scharf, C., Li, P., Muntwyler, J., Chugh, A., Oral, H., Pelosi, F., et al. (2005). Ratedependent AV delay optimization in cardiac resynchronization therapy. Pacing Clin. Electrophysiol. 28, 279-284. doi:10.1111/j.1540-8159.2005.40054.x.

Singh, J. P., Abraham, W. T., Chung, E. S., Rogers, T., Sambelashvili, A., Coles, J. A. J., et al. (2013). Clinical response with adaptive CRT algorithm compared with CRT with echocardiography-optimized atrioventricular delay: a retrospective analysis of multicentre trials. Eur. Eur. pacing, arrhythmias, Card. Electrophysiol. J. Work. groups Card. pacing, arrhythmias, Card. Cell. Electrophysiol. Eur. Soc. Cardiol. 15, 1622-1628. doi:10.1093/europace/eut107. 
Sohaib, S. M. A., Whinnett, Z. I., Ellenbogen, K. A., Stellbrink, C., Quinn, T. A., Bogaard, M. D., et al. (2013). Cardiac resynchronisation therapy optimisation strategies:

Systematic classification, detailed analysis, minimum standards and a roadmap for development and testing. Int. J. Cardiol. 170, 118-131.

doi:10.1016/j.ijcard.2013.10.069.

St. Jude Medical (2019). FREEDOM - A Frequent Optimization Study Using the QuickOpt Method. Available at: https://clinicaltrials.gov/ct2/show/results/NCT00418314 [Accessed August 11, 2020].

Sun, J. P., Lee, A. P.-W., Grimm, R. A., Hung, M.-J., Yang, X. S., Delurgio, D., et al. (2012). Optimisation of atrioventricular delay during exercise improves cardiac output in patients stabilised with cardiac resynchronisation therapy. Heart 98, 54-59.

doi:10.1136/heartjnl-2011-300278.

Walmsley, J., Arts, T., Derval, N., Bordachar, P., Cochet, H., Ploux, S., et al. (2015). Fast Simulation of Mechanical Heterogeneity in the Electrically Asynchronous Heart Using the MultiPatch Module. PLoS Comput. Biol. 11, e1004284. doi:10.1371/journal.pcbi.1004284.

Walmsley, J., Huntjens, P. R., Prinzen, F. W., Delhaas, T., and Lumens, J. (2016). Septal flash and septal rebound stretch have different underlying mechanisms. Am. J. Physiol. Heart Circ. Physiol. 310, H394-403. doi:10.1152/ajpheart.00639.2015.

Whinnett, Z. I., Davies, J. E. R. R., Willson, K., Chow, A. W., Foale, R. A., Davies, D. W., et al. (2006a). Determination of optimal atrioventricular delay for cardiac resynchronization therapy using acute non-invasive blood pressure. Europace 8 , 358-366. doi:10.1093/europace/eul017.

Whinnett, Z. I., R Davies, J. E., Willson, K., Manisty, C. H., Chow, A. W., Foale, R. A., et al. (2006b). Haemodynamic effects of changes in atrioventricular and interventricular delay in cardiac resynchronisation therapy show a consistent pattern: analysis of shape, magnitude and relative importance of atrioventricular and interventricular delay. Heart 92, 1628-1634. doi:10.1136/hrt.2005.080721.

Wish, M., Fletcher, R. D., Gottdiener, J. S., and Cohen, A. I. (1987). Importance of left atrial timing in the programming of dual-chamber pacemakers. Am. J. Cardiol. 60, 566571. doi:10.1016/0002-9149(87)90306-7. 



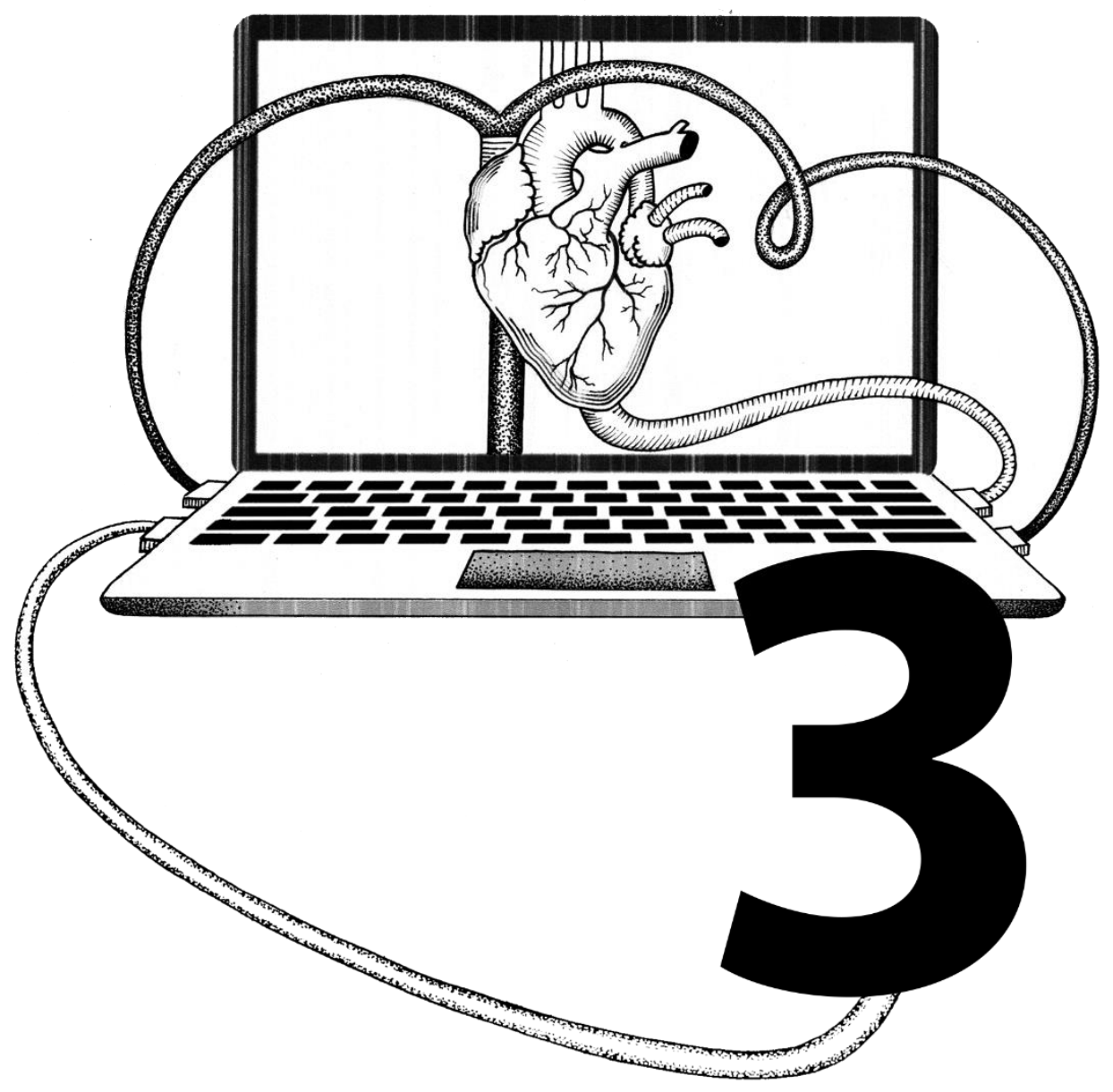




\section{Chapter 3}

\section{The left and right ventricles}

respond differently to variation of pacing delays in cardiac

\section{resynchronization therapy}

A combined experimentalcomputational approach

Erik Willemen, Rick Schreurs, Peter R. Huntjens, Marc Strik, Gernot Plank, Edward Vigmond, John Walmsley, Kevin Vernooy, Tammo Delhaas, Frits W. Prinzen, Joost Lumens

Frontiers in physiology vol. 10 17. 1 Feb. 2019, doi:10.3389/fphys.2019.00017 


\section{Abstract}

Introduction: Timing of atrial, right (RV) and left ventricular (LV) stimulation in cardiac resynchronization therapy (CRT) is known to affect electrical activation and pump function of the LV. In this study, we used computer simulations, with input from animal experiments, to investigate the effect of varying pacing delays on both LV and RV electrical dyssynchrony and contractile function.

Methods: A pacing protocol was performed in dogs with atrioventricular block ( $N=6)$, using 100 different combinations of atrial (A)-LV and A-RV pacing delays. Regional LV and RV electrical activation times were measured using 112 electrodes and LV and RV pressures were measured with catheter-tip micromanometers. Contractile response to a pacing delay was defined as relative change of the maximum rate of LV and RV pressure rise $\left(d P / d t_{\max }\right)$ compared to RV pacing with an A-RV delay of $125 \mathrm{~ms}$. The pacing protocol was simulated in the CircAdapt model of cardiovascular system dynamics, using the experimentally acquired electrical mapping data as input.

Results: Ventricular electrical activation changed with changes in the amount of LV or RV pre-excitation. The resulting changes in $\mathrm{dP} / \mathrm{dt}_{\max }$ differed markedly between the LV and RV. Pacing the LV 10-50ms before the RV led to the largest increases in LV dP/dt $t_{\text {max }}$. In contrast, RV dP/dt $t_{\text {max }}$ was highest with RV pre-excitation and decreased up to $33 \%$ with LV preexcitation. These opposite patterns of changes in RV and LV dP/dt $t_{\max }$ were reproduced by the simulations. The simulations extended these observations by showing that changes in steady-state biventricular cardiac output differed from changes in both LV and $\mathrm{RV} \mathrm{dP} / \mathrm{dt}_{\max }$. The model allowed to explain the discrepant changes in $\mathrm{dP} / \mathrm{dt}_{\max }$ and cardiac output by coupling between atria and ventricles as well as between the ventricles.

Conclusions: The LV and the RV respond in a opposite manner to variation in the amount of LV or RV pre-excitation. Computer simulations capture LV and RV behavior during pacing delay variation and may be used in the design of new CRT optimization studies. 


\section{Introduction}

Cardiac resynchronization therapy (CRT) is an established therapy for heart failure patients with a reduced left ventricular (LV) ejection fraction (EF) and left bundle branch block (LBBB) (Brignole et al., 2013). Through biventricular pacing, CRT aims to establish a more synchronous electrical activation of the ventricles and thereby improves cardiac pump function (Vernooy et al., 2007) and clinical outcome (Brignole et al., 2013). However, approximately one-third of the patients that receive CRT do not benefit from this therapy (Abraham et al., 2002; Auricchio and Prinzen, 2011; Cleland et al., 2005; Prinzen et al., 2013).

Programming of both atrioventricular (AV) and ventriculoventricular (VV) pacing delays strongly influences the contractile response to CRT, as determined by both ultrasound and maximum rate of LV pressure rise (LV dP/dt max $_{\text {) }}$ (Auger et al., 2013; Bogaard et al., 2012; Strik et al., 2013b). However, meta-analyses of multiple optimization methods showed that pacing delay optimization fails to provide long-term improvement in clinical outcome (Auger et al., 2013). Suggested reasons for the absence of long-term benefits of such optimization are that the default "out-of-the-box" delays are already fairly good and that the optimization methods employed are not accurate or robust enough. An alternative explanation could be that most pacing delay optimization strategies that have been developed solely take LV function into account. Right ventricular (RV) function is often overlooked, although several studies show that RV failure is an independent predictor of mortality in patients with LV failure with and without CRT (Groote et al., 1998; Ricci et al., 2017). Two clinical studies indicated that there was a poor correlation between the pacing delay settings providing the highest LV dP/dt $t_{\max }$ and RV dP/dt $t_{\max }$ values (Hyde et al., 2016; Sciaraffia et al., 2009). 
In recent years, computational models of cardiac electrophysiology, mechanics of the heart and cardiovascular system have increased our understanding of dyssynchronous heart failure and its treatment with CRT (Lee et al., 2018). Right ventricular function and its effect on CRT has however not been studied extensively using a computer modeling approach. Our group has been using the CircAdapt model of the heart and closed-loop circulatory system. While using a simplified cardiac anatomy, the advantages of this model are the inclusion of the entire (systemic and pulmonary) circulation and its high calculation speed (almost real time). In combination with experimental and clinical measurements, CircAdapt has shown to be able to identify and mechanistically understand the electromechanical substrates of the heart that are most responsive to CRT (Huntjens et al., 2018; Lumens et al., 2013, 2015).

A vast majority of the studies on the heart, also our aforementioned studies, are related largely to the LV. In the present study, we aim to study the changes in both LV and RV contractile response to variations of pacing delay settings in CRT, and to evaluate whether the CircAdapt computer model reliably simulates LV and RV pump function during these interventions. Subsequently we aim to use the computer modeling results to investigate how cardiac output is affected by differences between LV and RV contractile changes.

\section{Methods}

\section{Animal experiments}

- Animal handling was performed according to the Dutch Law on Animal Experimentation and the European Directive for the Protection of Vertebrate Animals Used for Experimental and Other Scientific Purpose. The protocol was approved by the Animal Experimental Committee of Maastricht University. The animal experimental methodology has been partially published elsewhere (Strik et al., 2013a). In short, 6 adult mongrel dogs were anesthetized 
using midazolam $(0,25 \mathrm{mg} / \mathrm{kg} / \mathrm{h})$ and sufentanyl $(3 \mu \mathrm{g} / \mathrm{kg} / \mathrm{h})$ and a complete AV-block was induced by radiofrequency ablation. The dogs received pacing electrodes in the right atrium (A), RV apex and epicardially on the basal posterolateral wall via a left-sided thoracotomy.

- Measurements were performed 12-21 weeks after inducing the AVblock using an external custom-built pacing system. RV-only pacing with an $A$ to RV (A-RV) pacing delay of $125 \mathrm{~ms}$ was used as the baseline pacing setting, mimicking the activation pattern as seen in LBBB. The A-RV and A to LV (A-LV) delays were then programmed individually, ranging from 50 to $230 \mathrm{~ms}$ in steps of $20 \mathrm{~ms}$, resulting in 100 possible combinations (Figure 1A). Pacing delay settings were assigned in a random order and baseline recordings were repeated after every $5^{\text {th }}$ setting. During each setting, continuous, invasive hemodynamic and electrocardiographic measurements were performed (Figure 1B). 7F catheter-tip manometers were used for LV and RV pressure measurement. Epicardial electrograms of the LV free wall (LVFW) and RV free wall (RVFW) were recorded from 2 multielectrode custom-made bands holding 102 contact electrodes. Septal endocardial electrograms were recorded from two multielectrode catheters with 7 contact electrodes on the RV septum and 3 on the LV septum. Measurements were recorded for a minimum of two respiratory cycles at each pacing delay setting.

\section{Data analysis}

Local electrical activation times were determined as the duration between the atrial pace and the timepoint of steepest negative deflection of the electrogram using custom software (Figure 1B, green line in local EGM's). For quantification of intraventricular electrical dyssynchrony we calculated total activation time as the difference between earliest and latest activation time of the RVFW (RV TAT) and of the whole LV (LV TAT). The latter was calculated from the combination of epicardial LVFW and endocardial septal electrodes. To quantify electrical interventricular dyssynchrony we used the ventricular electrical uncoupling (VEU) index. VEU was defined as the difference between mean LVFW and mean RVFW activation times (Ploux et al., 2013). 
For both the LV and the RV, response to pacing was defined as the relative change in $\mathrm{dP} / \mathrm{dt}_{\max }$ compared to the baseline setting. We applied quadratic LOESS fitting to account for measurement variability within each dog (Cleveland, 1979). Furthermore, in order to quantify a generic canine response to changes in pacing delay, we created a single representative canine dataset by taking the mean values of the dogs for each setting. We also applied linear 2D interpolation between the measurements in the heat map visualizations of all pacing delay settings. All these calculations were performed in Matlab 2016A (The Mathworks Inc, Natick MA, USA).

\section{Computer simulations}

The CircAdapt model of the entire heart and circulation (Arts et al., 2005; Lumens et al., 2009; Walmsley et al., 2015a), which can be downloaded from www.circadapt.org, was used to simulate cardiovascular mechanics and hemodynamics during pacing delay variations as applied in the animal experiments. Previous experimental and clinical studies have shown that the CircAdapt model realistically relates local ventricular myofiber mechanics to global cardiovascular hemodynamics in dyssynchronous and paced hearts(Huntjens et al., 2014; Leenders et al., 2012; Lumens et al., 2015; Walmsley et al., 2015a, 2015b).

The source code of the CircAdapt model used for all simulations as well as the Matlab ${ }^{\circledR}$ (The MathWorks, Natick, MA) scripts to perform all simulations and analysis are provided as an online data supplement. In summary, the CircAdapt model is a reduced order model of the human four-chamber heart connected to a closed-loop cardiovascular system, with lumped pulmonary and systemic circulations. It uses a simplified ventricular geometry, where cardiac walls are represented by thick spherical shells consisting of contractile myocardium. The MultiPatch module enables cardiac walls to be subdivided into an arbitrary number of wall segments (patches). Tissue properties and activation time can 
differ between patches, but all patches within a wall share a common wall tension and curvature. Since wall tension is the same in all patches within a wall, spatial location within a wall is not required to calculate deformation in a patch.

In CircAdapt wall tension and curvature determine cavity pressure through Laplace's law (Lumens et al., 2009; Walmsley et al., 2015a) Fiber stress in a patch is the sum of an active component, representing myofiber contraction, and a passive component. The active stress component incorporates length-dependence of the force generated and the duration of contraction. The passive component provides a nonlinear relationship between myofiber stress and strain. More details on the phenomenological model of myocardial contraction and the validation of the MultiPatch module are previously published by Walmsley et al. (Walmsley et al., 2015a). 


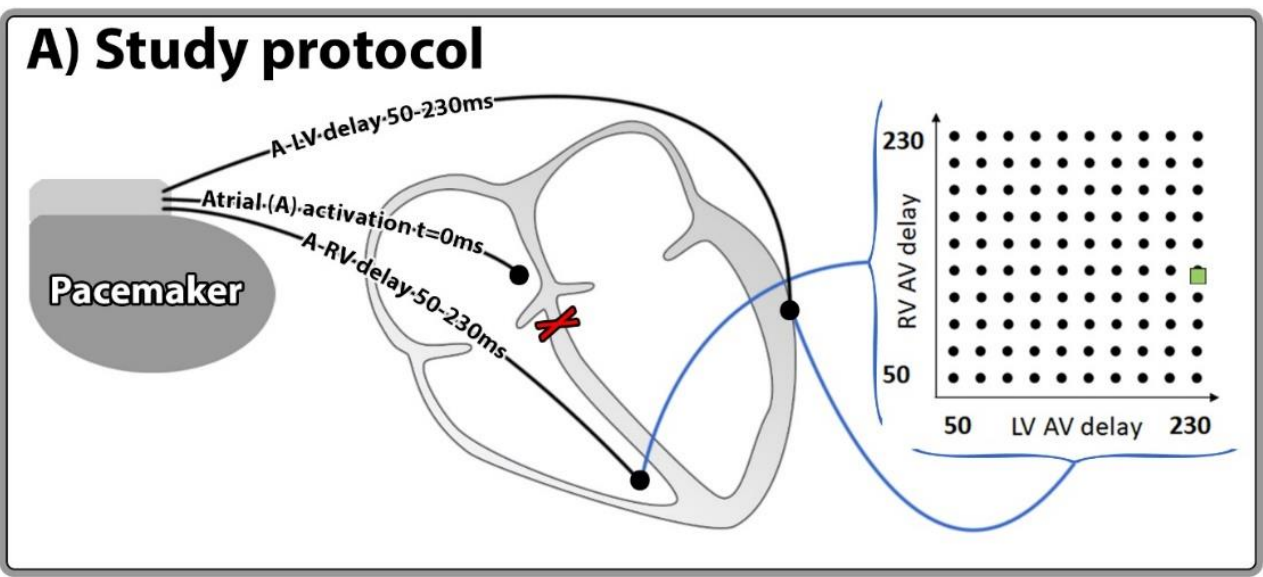

\section{B) Measurements}

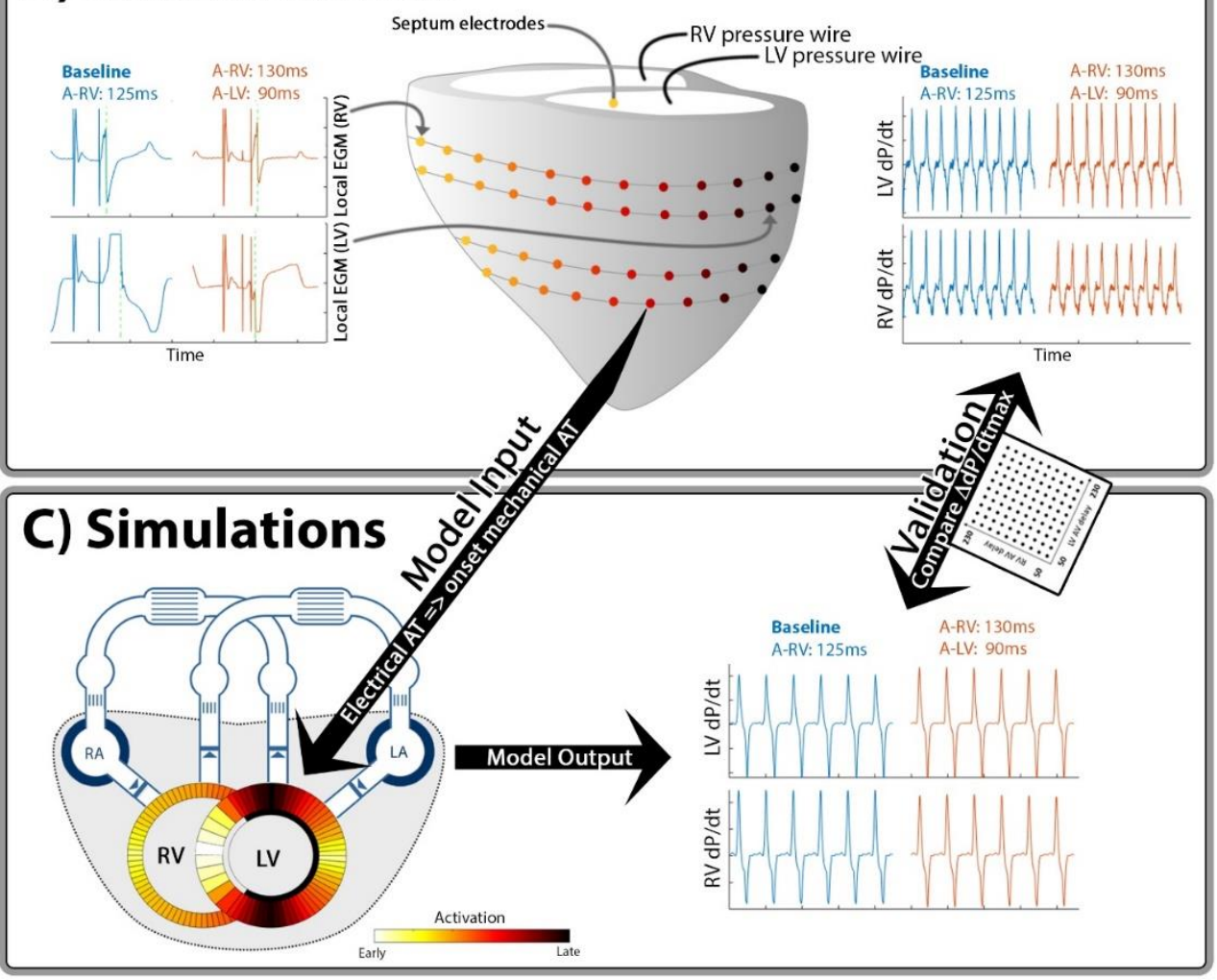


$\leftarrow$ Figure 1 -Schematic representation of the methods used in this study. 100 different A-LV/A-RV pacing delay combinations were programmed $(A)$ while pressures and local electrical activation were measured (B). Generic activation maps, derived from local electrograms, were used as onset of mechanical activation in the computer simulations (C). The resulting output of the simulations and measurements was compared for validation purposes. The green square in the heat maps indicates the baseline pacing setting.

\section{Simulating a healthy human reference heart and circulation}

Cardiac adaptation implemented in the CircAdapt model was used to obtain a reference parameterization that represents a healthy human cardiovascular system (Arts et al., 1994, 2005, 2012). The tissue volumes and areas in the cardiac walls and large blood vessels were adapted as described previously (Arts et al., 2005, 2012). A resting cardiac output of $5.1 \mathrm{l} / \mathrm{min}$ and heart rate of $70 \mathrm{bpm}$ were assumed. Cardiac output was tripled and the heart rate was doubled during the stress-state of the adaptation process. Mean arterial pressure (MAP) was maintained at $92 \mathrm{mmHg}$ during the adaptation process. The resulting reference simulation was used as the basis for subsequent pacing simulations.

\section{Using electrical activation to simulate the pacing delay optimization protocol}

We divided the ventricular wall in the same amount of segments as the number of available electrodes (52 LV free wall, 50 RV free wall and 10 septal segments). Time of onset of activation was assigned based on the electrical activation times measured in the animal experiments.. As previously stated, in the current MultiPatch module the segments were considered to be mechanically coupled in series, meaning that the order in which patches were placed was not significant (Walmsley et al., 2015a). This allowed sorting of the activation times per wall in each measurement, before taking the median of the dogs, to get a generic activation pattern. The benefit of this generic activation pattern is that it was less affected by differences in band placement, heart size and electrodes with insufficient contact in the dogs. 
A representative baseline simulation was obtained by imposing the ventricular activation pattern measured during the experimental baseline condition, i.e. RV-only pacing with an A-RV delay of $125 \mathrm{~ms}$. Systemic vascular resistance was adapted to obtain a MAP of $60 \mathrm{mmHg}$ and heart rate was set to $80 \mathrm{bpm}$, both similar to the animal experiments. Furthermore, total circulating blood volume was adjusted so that cardiac output was maintained at $5.1 \mathrm{~L} / \mathrm{min}$. The resulting baseline simulation was used as the starting point for the pacing setting simulations. For each of the 100 pacing delay simulations, the pattern of ventricular activation was changed to the activation pattern measured in the canine experiments and the resulting beat-to-beat changes in ventricular mechanics and hemodynamics were stored until a new hemodynamic steady state was reached. During all pacing simulations, systemic vascular resistance and total circulating blood volume were kept constant in order to quantify the acute effect of pacing-induced changes of ventricular pump mechanics and cardiac hemodynamics. Simulated steady-state $\mathrm{dP} / \mathrm{dt}_{\max }$ values are compared with the experimental measurements. In addition, the simulations extended the animal experiments by providing quantitative insight in the beat-to-beat and steady-state changes of ventricular volumes and cardiac output. 


\section{Results}

Baseline characteristics for the AV-blocked dogs are described in Table 1.

\begin{tabular}{|c|c|}
\hline & $\begin{array}{l}\text { During baseline RV-only } \\
\text { pacing }\end{array}$ \\
\hline Weight (kg) & $19.8(19.4-21.4)$ \\
\hline $\mathrm{MAP}(\mathrm{mmHg})$ & $55(42-71)$ \\
\hline Systolic Arterial Pressure (mmHg) & $70(62-81)$ \\
\hline Diastolic Arterial Pressure $(\mathrm{mmHg})$ & $45(31-53)$ \\
\hline $\mathrm{LV} d \mathrm{~d} / \mathrm{dt}_{\max }(\mathrm{mmHg} / \mathrm{s})$ & $1205(1183-1646)$ \\
\hline $\mathrm{RV} d \mathrm{P} / \mathrm{dt}_{\max }(\mathrm{mmHg} / \mathrm{s})$ & $520(345-700)$ \\
\hline $\begin{array}{l}\text { Weeks between AVB and Sacrifice } \\
\text { (weeks) }\end{array}$ & $13(12-21)$ \\
\hline
\end{tabular}

\section{Electrical effects of altering pacing delay settings}

Figure 2 shows the typical examples of electrical activation patterns acquired using contact mapping in a dog with AV block during LV preexcitation, simultaneous RV and LV pacing and RV pre-excitation. In case of extreme pre-excitation, capture in the last paced ventricle was lost due to activation via the contralateral ventricle (indicated by the gray line). RV pre-excitation led to the largest LV TAT while LV pre-excitation resulted in an increase of RV TAT. During simultaneous pacing, two wave fronts originating from the RV and LV pacing electrodes fused and resynchronized the heart as indicated by a decrease in LV TAT. 


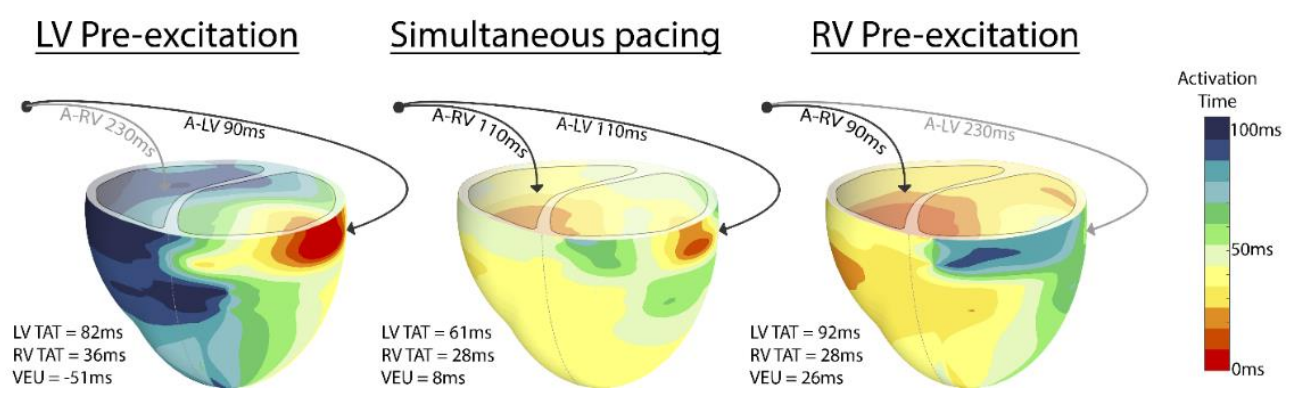

Figure 2 - Epicardial electric activation maps in a paced dog heart with complete AV block during LV pre-excitation (left), simultaneous pacing (middle) and $R V$ pre-excitation (right). Black arrows indicate capture, whereas grey arrows indicate loss of capture.

Figure 3 shows the changes in electrical dyssynchrony indexes in dogs with variation of pacing delay settings. There was no change in LV and RV TAT and VEU when changing the AV delay during simultaneous activation of the LV and RV (left column). Both LV and RV TAT (first 2 rows of Figure 3 ) were lowest during simultaneous $R V+L V$ pacing. The RV showed the largest TAT during LV pre-excitation or LV-only pacing (upper left corners in the heat maps). LV TAT showed a relatively large increase with large RV pre-excitation (right side of middle panels and lower right corners in heat maps), while RV TAT did not increase much. As indicated by the VEU (bottom row figure 3), during LV-only pacing the LVFW was activated more than $40 \mathrm{~ms}$ before the RVFW. During RVonly pacing the LVFW was, on average, activated more than $20 \mathrm{~ms}$ later than the RVFW. The LVFW and RVFW were activated simultaneously with very slight LV pre-excitation (Figure 3 bottom right, $V E U=0$ ).

\section{Hemodynamic effects of altering pacing delay settings}

The changes in LV and $\mathrm{RV} d \mathrm{P} / \mathrm{dt}_{\max }$ in response to changes in pacing delay settings are presented in figure 4 (top and bottom, respectively). Increasing AV delay during simultaneous RV and LV pacing (left column and identity line in the heat maps) hardly affected LV and RV dP/dt $t_{\max }$ in both measurements and simulations. The relative effect of changing $\mathrm{V} V$ delay (green, second column from left) was largest in $\mathrm{RV} \mathrm{dP} / \mathrm{dt}_{\text {max }}$. 
Changing pacing settings from RV to LV pre-excitation decreased RV $\mathrm{dP} / \mathrm{dt}_{\max }$ with more than $30 \%$ in the experiment. The decrease in RV $\mathrm{dP} / \mathrm{dt}_{\max }$ was less pronounced in the simulations but followed the same pattern. LV dP/dt $\max$ was highest with LV pre-excitation and simultaneous pacing in both the experiment and simulations.

The heat maps of both the animal experiments and computer simulations (right side of figure 4) show a qualitatively similar pattern where the largest changes in both LV and $\mathrm{RV} \mathrm{dP} / \mathrm{dt}_{\max }$ are observed

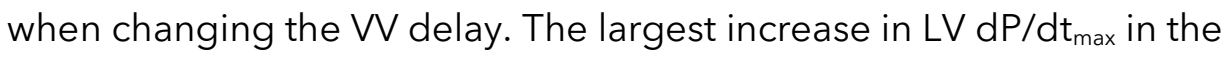
measurements was reached with a short A-LV $(50 \mathrm{~ms})$ and A-RV $(90 \mathrm{~ms})$. $\mathrm{LV}$ pre-excitation led to a larger increase in $\mathrm{LV} d P / d t_{\max }$ than $\mathrm{RV}$ preexcitation during all measurements and simulation, with an optimal LV pre-excitation range of $10-50 \mathrm{~ms}$. For $\mathrm{RV} \mathrm{dP} / \mathrm{dt}_{\max }$ all LV pre-excitation pacing settings led to a decrease up to $33 \%$ in the experiment and $18 \%$ in the simulations, while RV pre-excitation caused little change compared to baseline (RV-only) pacing. 


\section{effect of changing \\ AV delay}

\section{Increase in AV delay}

A-LV: $50 \quad 90 \quad 130 \quad 170 \quad 210$

A-RV: $\quad 50 \quad 90 \quad 130 \quad 170 \quad 210$
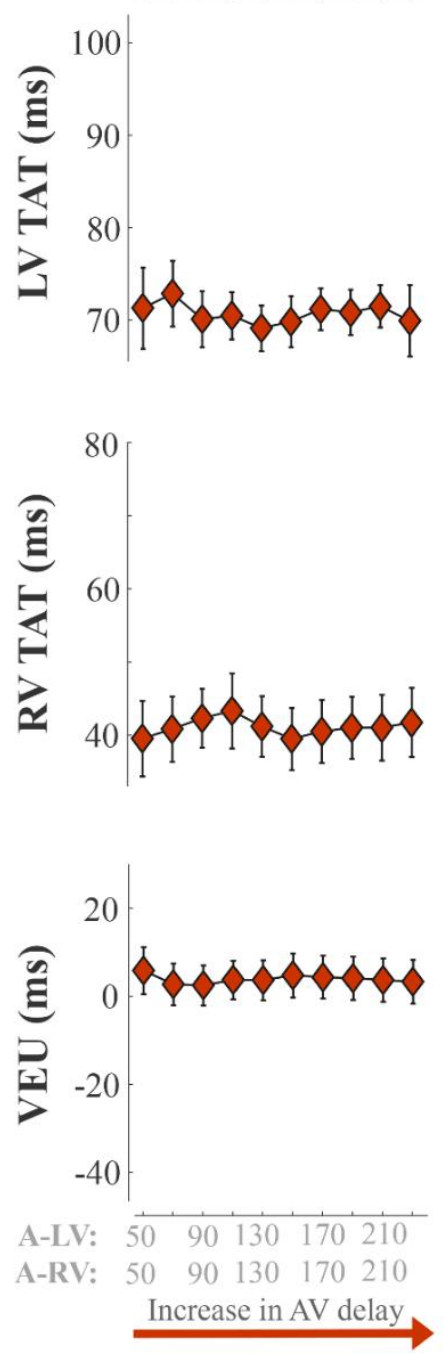

effect of changing

VV delay

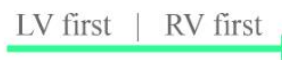

$50 \quad 90 \quad 130170210$ (ms) $210 \quad 170 \quad 130 \quad 90 \quad 50 \quad$ (ms)
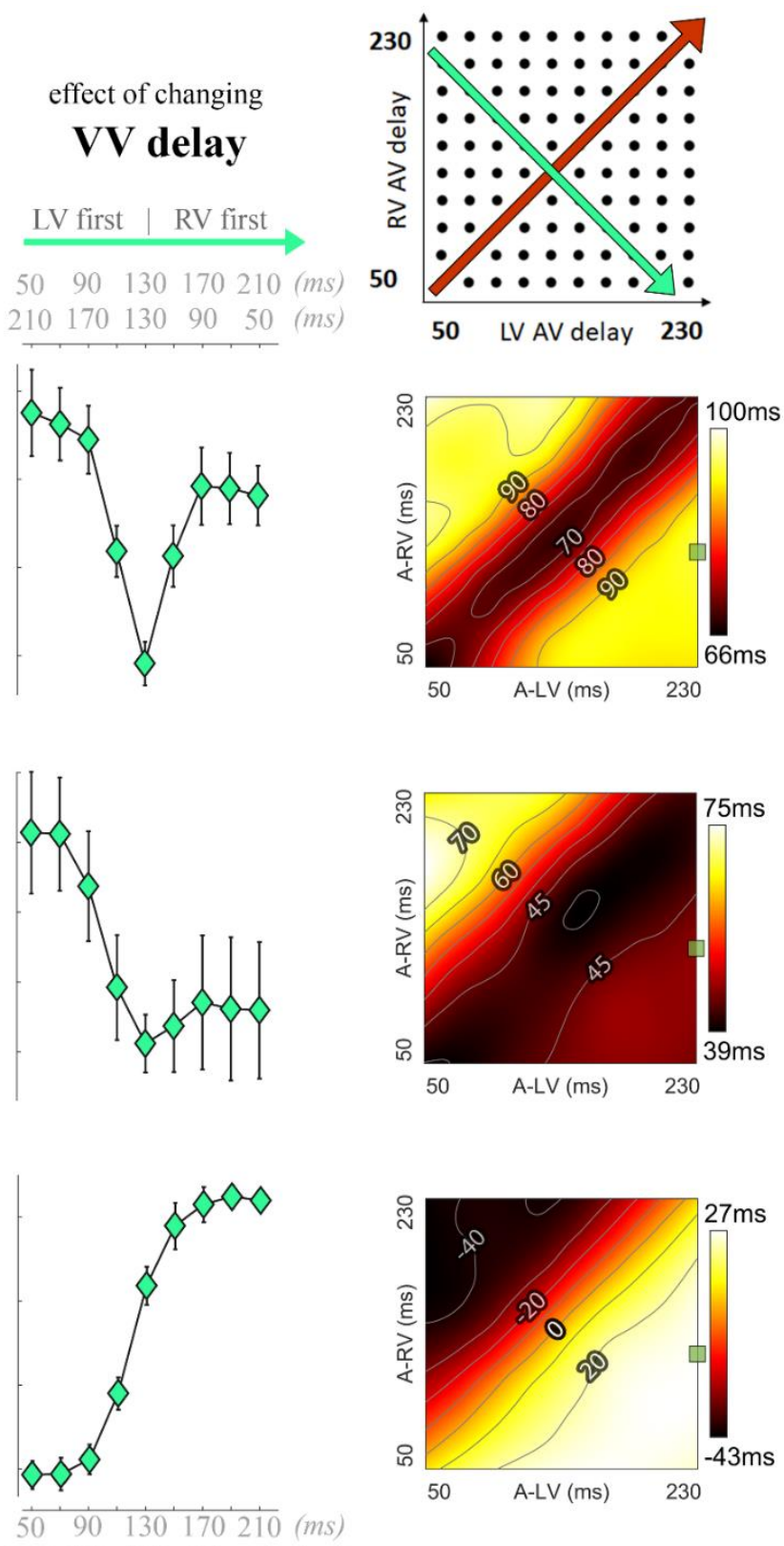

$\begin{array}{llllll}210 & 170 & 130 & 90 & 50 & \text { (ms) }\end{array}$ LV first | RV first 
$\leftarrow$ Figure 3 -Changes in electrical dyssynchrony indices during variation in pacing delay in the animal experiments. The left column shows the effect of increasing AV delay during simultaneous $R V+L V$ pacing; the middle column shows changes in $V$ delay (green, from $L V$ pre-excitation to $R V$ pre-excitation) and the heat maps on the right are the results for all pacing setting (mean of 6 dogs, bars represent standard errors of the mean). From top to bottom: Total activation time (TAT) of the total LV (free wall and septum), RV free wall (RVFW) and VEU (Ventricular Electrical Uncoupling). The green square in each heat map indicate the baseline pacing setting.

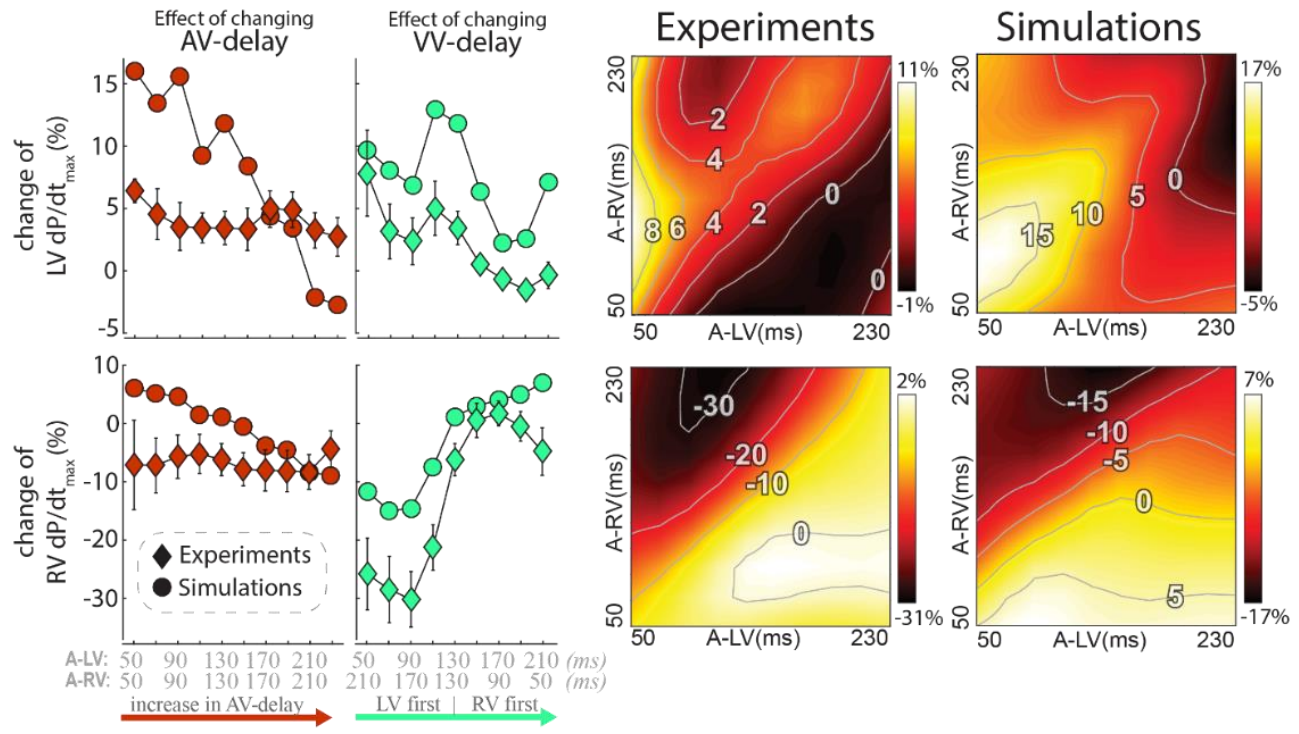

Figure 4 - Changes in contractile response as a result of changes in pacing delays in experiments and simulations. Percentile change from baseline of $L V$ $d P / d t_{\max }$ (top) and $R V d P / d t_{\max }$ (bottom). The left rows depict the same AV and $W$ delay settings as in Figure 3 are shown. Heat maps for both the experiment ( $3^{\text {rd }}$ column) and simulations ( $4^{\text {th }}$ column). Diamonds: Canine measurements (Mean (standard error of the mean) of 6 dogs; Circles: Simulation output. 


\section{Changes in simulated cardiac output at different pacing delays}

While $d P / d t_{\max }$ values are regarded as a measure of ventricular contractility, cardiac output may be more closely related to pump function of the entire heart. Note that due to the closed loop circulation, in a steady state situation cardiac output of the RV and LV are the same. Cardiac output was not determined in the experiments, but it was calculated in the model simulations. In these simulations the changes in cardiac output following a switch in pacing delay differed from the changes in both LV and RV dP/dtmax (Figure 5). Cardiac output was more sensitive to changes in AV-delay than to changes in VV-delay. AV-delays of 50 and $70 \mathrm{~ms}$ led to the largest increases in cardiac output, amounting up to $9 \%$.
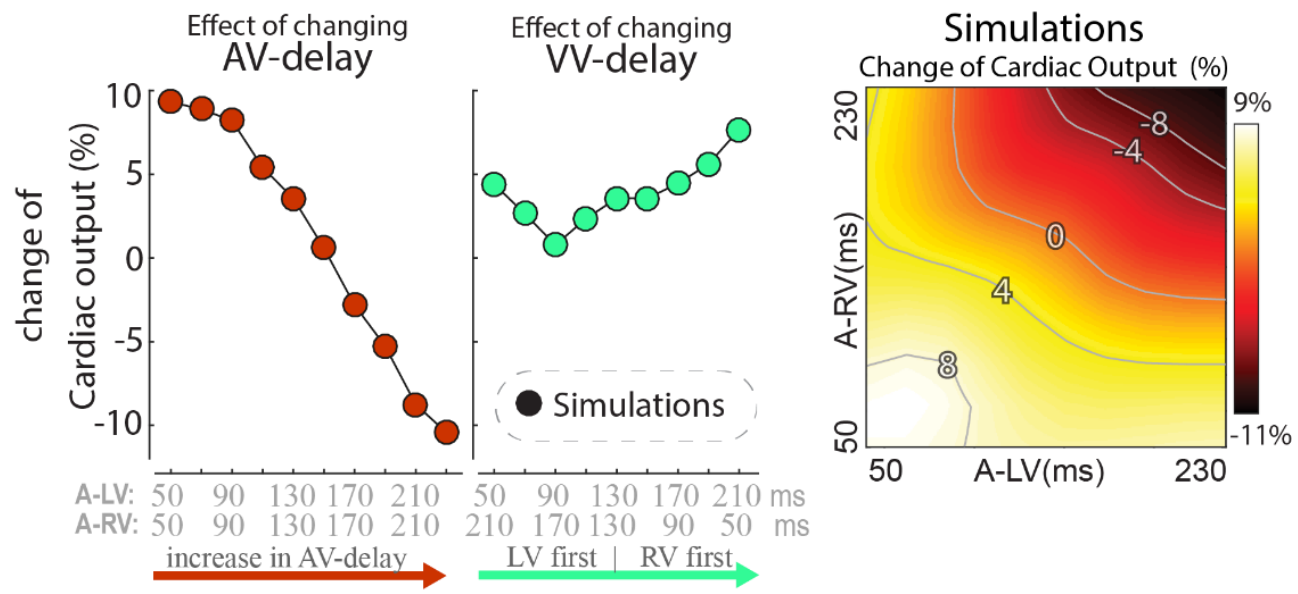

Figure 5 - Relative change in simulated steady-state cardiac output with a change in pacing delay settings. Depicted are the changes relative to baseline (see text).

In order to find an explanation for the differences in behavior between cardiac output and RV and LV dP/dt $t_{\max }$ we compared the time course of these parameters as well as EDV during the first beats after start of a certain setting, in this case LV pre-excitation (Figure 6). In the first beat after the change in pacing setting and therefore also activation sequence (see above), both LV stroke volume and $\mathrm{dP} / \mathrm{dt} \mathrm{t}_{\max }$ increased while RV stroke volume and $\mathrm{dP} / \mathrm{dt}_{\max }$ decreased. In the subsequent beat 
RV EDV increased, due to the smaller SV of the previous beat, whereas LVEDV decreased. As a consequence of these EDV changes, RV SV recovered and LV SV decreased to some extent and in the third and subsequent beats an steady state (SS) was reached, with SV in both ventricles (and therefore cardiac output) increasing by about $3 \%$. This example, representative for the other conditions, illustrates that $\mathrm{dP} / \mathrm{dt}_{\max }$ was largely independent of preload, whereas SV depended on it, most likely due to the length dependent activation, implemented in the CircAdapt model (see methods).

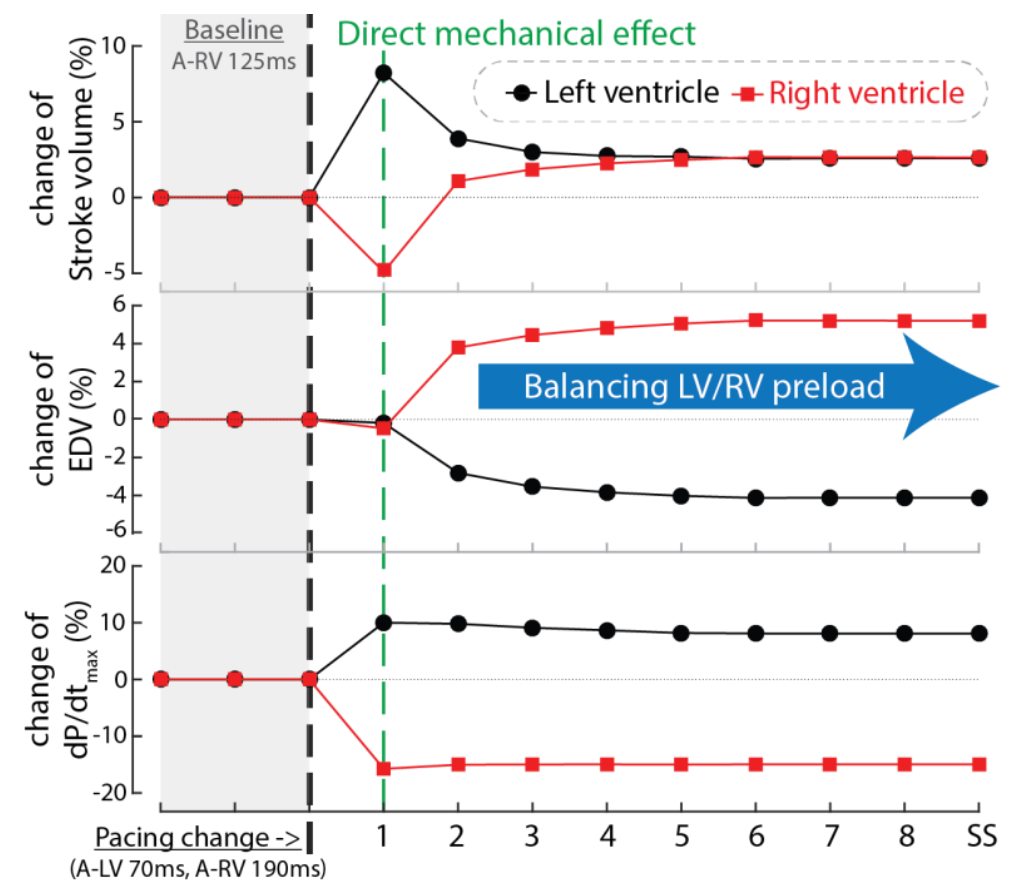

Figure 6 - Time courses of the relative change of stroke volume (top), enddiastolic volume (EDV, mid) and $d P / d t_{\max }$ of the $L V$ (black circles) and $R V$ (red squares) after changing pacing delay from baseline (A-RV 125ms) to LV preexcitation (A-LV 70ms, A-RV 190ms) in computer simulations. The black dashed line indicates the start of the change in pacing delay. The numbers in the blue bar indicate the number of simulated cardiac cycles. SS=steady state. 
Figure 7 shows the response of stroke volume of the LV and RV after simulated programming of nine different pacing settings. In the first beat RV SV remained either unchanged or decreased as compared to baseline, indicating little direct mechanical benefit of the change in pacing delay for the RV. However, similar to the example in figure 6, copied into the left upper panel of figure 7, RV stroke volume changed considerably in subsequent beats. While changes in LV SV initially differed from RV SV, a steady state was reached after several simulated beats. Note that the largest benefit in SV, and therefore cardiac output, was primarily dependent on AV-delay. For example, in the bottom row (A-RV 70ms) the optimized RV filling improved stroke volume to such a degree that, through the serial coupling, LV preload increased, leading to a further increase in LV stroke volume after the second cycle. On the other hand, at longer AV-delays this atrial-ventricular coupling decreases, resulting in a lower steady-state cardiac output. 

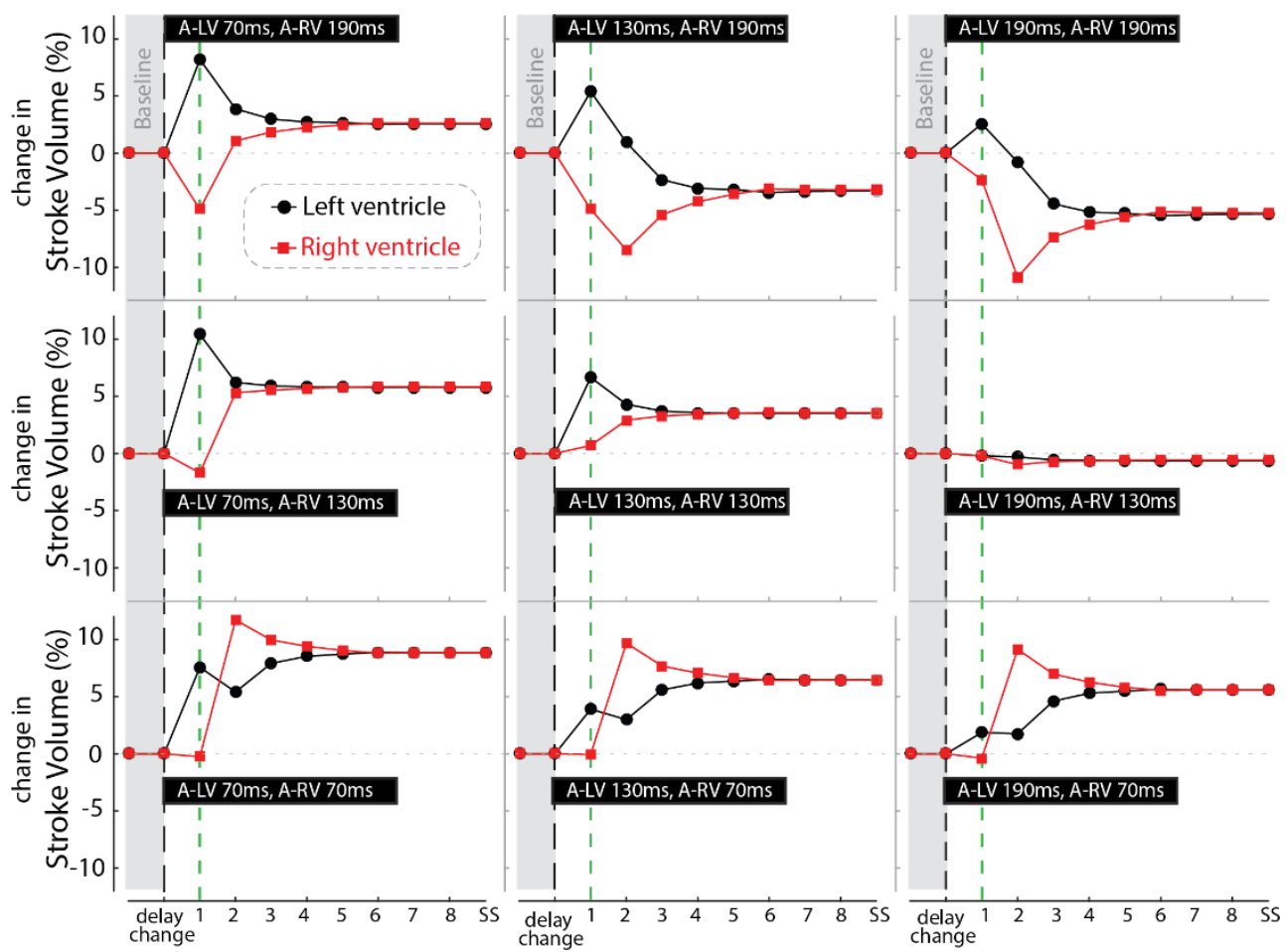

Figure 7 - Relative change in stroke volume of the left (black circles) and right (red squares) ventricle over a number of simulated cycles until steady-state (SS) after changing pacing delay (the 9 settings shown in the black bars). Black dashed line indicates the moment of changing the pacing delays while the green line marks the first beat. 


\section{Discussion}

In this study we investigated the influence of LV and RV pacing delay settings on LV and RV electrical activation and contractility in animal studies and computer simulations. Both studies showed that LV TAT is smallest during synchronous RV and LV stimulation and increases when V delays increase. RV TAT becomes larger in particular during LV preexcitation. LV and RV contractility vary most, and in opposite direction, with changes in $\mathrm{V}$ delay settings. After demonstrating the realistic simulations in the model, we used the model to calculate cardiac output changes and to explain why changes cardiac output differed from both RV and LV contractility. The latter findings demonstrate how a model like CircAdapt can extend mechanistic understanding of circulatory changes due to a device therapy.

\section{LV and RV contractility respond in opposite manner to variations in} VV pacing delays

A key finding in the present study is that LV and $R V d P / d t_{\max }$ change in opposite direction when changing the $\mathrm{V}$ pacing delay. Sciaraffia et al previously demonstrated that RV and LV dP/dtmax identify different 'optimal' VV delays in most of the patients included in their study (Sciaraffia et al., 2009). Furthermore, Houston et al recently showed that in patients with dyssynchronous heart failure, RV-only pacing leads to higher RV dP/dtmax than LV-only or simultaneous LV+RV pacing (Houston et al., 2018). In an experimental study in pigs, different $V$ delays were tested at different pacing locations (Quinn et al., 2006). Similar to our study, these investigators found that RV pre-excitation led to a higher RV $\mathrm{dP} / \mathrm{dt}_{\max }$ than LV pre-excitation. The fact that findings were consistent in patients, animals and a computer model implies that the opposing changes in hemodynamics, caused by varying $\mathrm{W}$ pacing delays, are caused by a universal mechanism. 
Another key finding is that ventricular specific pre-excitation is required for a good contractile function in both the LV and RV. This is illustrated

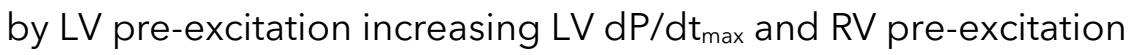
leading to the largest $\mathrm{RV} d P / d t_{\max }$ values. In contrast, changes in $\mathrm{AV}$ delay have less effect on measured and simulated LV and RV dP/dt $t_{\max }$. In a previous study, in which we evaluated the relative importance of interventricular and intraventricular dyssynchrony for contractile

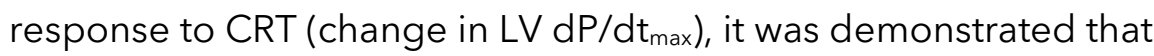
interventricular dyssynchrony during intrinsic rhythm is the dominant electrical substrate driving response to CRT (Huntjens et al., 2018). In contrast, intraventricular dyssynchrony showed little effect on LV $\mathrm{dP} / \mathrm{dt}_{\max }$, which is in line with experimental observations that reducing LV TAT by multipoint pacing does not improve LV dP/dt $\mathrm{t}_{\max }$ (Ploux et al., 2014). However, results in this study suggest that intraventricular dyssynchrony might still play a modulating role since increase in LV TAT, with large LV pre-excitation, led to a decrease in LV dP/dtmax compared to slight LV pre-excitation. Because RV TAT increases concurrently with decreases in VEU we cannot distinguish if either intra- or interventricular dyssynchrony has a larger effect on RV contractility.

In agreement with our observations, other studies in patients have shown that the lowest electrical dyssynchrony does not necessarily lead to the highest LV contractility or better clinical outcome (Lumens et al., 2013; Thibault et al., 2011). Optimization based on minimization of electrical dyssynchrony alone might therefore not be sufficient. Even if the optimal electrical activation for LV contractility is known, further clinical studies are required to investigate whether the gain in LV contractility outweighs the loss of RV contractility, especially since cardiac output might not match either or both but be a combination/compromise. 


\section{CircAdapt simulations capture both $\mathrm{LV}$ and RV contractile response to pacing delay changes}

The present study demonstrates that the CircAdapt model can capture pacing-induced changes to both LV and RV contractile function. It is especially the response of the RV to pacing that has been less well studied, both by our group and by others.

The use of experimental measurements electrical activation of the paced dog heart, derived from canine experiments, coupled to the simulation of the entire circulation, resulted in changes in RV and LV dP/dtmax that closely mimicked values measured in dog experiments.

Earlier studies have shown that CircAdapt enables realistic simulation of cardiac response to CRT, mostly focusing on LV function (Huntjens et al., 2018; Lumens et al., 2013, 2015). In one of those studies, it was demonstrated that the RV plays an important role in the improvement of LV function during LV-only pacing (Lumens et al., 2013). The present study extends the mechanistic insight in the working action of CRT in the context of pacing delay optimization, where the complex mechanical and hemodynamic interactions between the four cardiac cavities and the surrounding circulations are found to be important.

As demonstrated in this study, the CircAdapt model can capture the complexity of different LV and RV responses to CRT by incorporating several relevant components of cardiovascular interaction. Firstly, it realistically incorporates direct mechanical interaction between the three ventricular walls (Lumens et al., 2009). Secondly, it allows realistic simulation of regional myocardial mechanics in the ventricular walls of the asynchronously activated heart (Leenders et al., 2012; Walmsley et al., 2015a), thereby enabling experimentally measured activation times to be imposed and the related intraventricular heterogeneities in mechanical myofiber behavior to be simulated. Thirdly, it is a closedloop system allowing for indirect (serial) hemodynamic interaction between the left and right side of the heart through the systemic and 
pulmonary circulations (Arts et al., 2005; Lumens et al., 2010). Fourthly, its four-chamber heart captures the dynamics of hemodynamic atrioventricular interactions (Jones et al., 2017). Lastly, it includes the mechanical interaction through pericardial constraint, with an increase in the volume of a chamber altering the pressure in the other chambers and, hence, diastolic filling and septal position (Palau-Caballero et al., 2017).

\section{Simulation-derived mechanistic insights}

The ability of CircAdapt to realistically simulate both LV and RV hemodynamics during pacing allowed us to further study the impact of differences between both chambers on cardiac output. In particular, the model showed a difference in response between $\mathrm{dP} / \mathrm{dt}_{\max }$ and SV/CO. Similar differences have been observed in a clinical study where LV $\mathrm{dP} / \mathrm{dt}_{\max }$ responses differed from responses in stroke work (van Everdingen et al., 2018).

Our simulations provided a plausible explanation for this paradoxical observation. While LV and RV stroke volume of the first beat after a change of pacing delay can differ substantially, a common steady-state stroke volume and thereby cardiac output is reached due to balancing ventricular preload conditions during the next few beats. There are three main determinants of the newly achieved steady-state preload condition. First, the changes in stroke volumes directly change the endsystolic volumes and thereby the end-diastolic volumes in the next beat. Second, the pacing-induced changes in effective left and right AV delays change the efficiency of atrial and ventricular diastolic filling and subsequent systolic contraction. Thirdly, changes in stroke volume affect the filling of the other ventricle through the systemic and pulmonary circulations. 
While $d P / d t_{\max }$ changes congruently with first-beat stroke volume in the same ventricle, it is less affected by changes in preload. As a result, LV and $\mathrm{RV} d \mathrm{P} / \mathrm{dt}_{\max }$ are much more sensitive to changes of $\mathrm{V}$-delay and, hence, asynchrony of electrical activation than to changes of AV-delay. On the other hand, changes of AV-delay affect cardiac output more than $L V$ and RV dP/dt $t_{\text {max }}$.

\section{Computer modeling in therapy optimization}

Other cardiac computer modelling studies have been conducted to investigate other factors in the optimization of CRT therapy. For example, in a cohort of 648 virtual patients it was found that the location of the LV pacing site is an important factor in response to CRT (Crozier et al., 2016). Electrophysical cardiac computer modeling studies also demonstrated the importance of LV pacing site and the potential of simulations to predict the electrical optimal pacing location and setting (Miri et al., 2009; Niederer et al., 2011; Reumann et al., 2007). Our study shows, however, that an electrical optimum (lowest electrical dyssynchrony) might not necessarily be optimal for overall pump function.

The CircAdapt simulations performed for this study can run on a single core in real time. CircAdapt requires activation time as input and lacks the cardiac electrophysiological model necessary to extract this information from standard clinical data. This input could, however, potentially be generated by other models, for example the ones referred to in the previous paragraph. This would also allow for testing of alternative pacing sites, which would result in different activation patterns, which subsequently can be used as input for CircAdapt simulations. A workflow where fast and anatomically realistic cardiac electrophysiological simulations are combined with cardiac mechanical and circulatory CircAdapt simulations might further increase the clinical applicability of cardiac computer models. 


\section{Study Limitations}

In this study the pacing experiments were performed in relatively healthy canine hearts. Previous work from our group showed that chronic total AV-block leads to structural changes (hypertrophy) and electrical remodeling (QT-time prolongation), but normal contractility (Peschar et al., 2003). On the other hand, patients treated with CRT have different levels of myocardial remodeling and heart failure, which may affect the response to pacing (Nguyên et al., 2018). Results of this study might differ from patient data since long-term structural remodeling was not included in both the animal and computational experiments. On the other hand, differences in RV and LV response observed in this study could mean that different pacing settings might affect the positive and/or negative remodeling of the LV and RV.

A major difference between this animal experiment and patients in dayto-day life is that the animals were anesthetized. To allow comparison between the experiments and simulations, the model's LV afterload in the baseline situation was adapted to fit the measured mean arterial pressure in the dogs. After the change in activation delays regulation was disabled, which is likely similar to the anesthetic condition were regulation is slow. Caution should however be taken when translating results of this study towards the clinical setting considering that loading conditions potentially affect the effect of pacing delay changes (Quinn et al., 2009). Future studies are needed to investigate how loaddependent the observed effects of pacing delay optimization are and how homeostatic regulation interacts with changes in LV and RV contractility. A final limitation of the CircAdapt (and most other computer models in this field) is that changes in function of the autonomic nervous system are not taken into account. 


\section{Clinical perspective}

The results of this study raise the question what outcome measure is best to use for optimization of pacing delay. Current clinical practice focusses almost exclusively on the LV, using parameters like LV dP/dt $t_{\text {max }}$ aortic outflow integral, and LV systolic (or aortic) pressure. This study demonstrates that improving LV function can reduce RV function. Furthermore, cardiac output is not necessarily increasing when LV $\mathrm{dP} / \mathrm{dt}_{\text {max }}$ increases. Hence, an exclusive focus on the LV might not lead to the best overall outcome. Therefore, future studies on optimization of therapy should not exclusively focus on the LV but also include measures of RV and/or whole heart function. In our analysis of cardiac output we also demonstrated that the moment of measurement affects what physiological phenomenon is actually observed. Contractile function alone might better be observed in the first beats while longer lasting measurements, that allow reaching a steady state, will provide more information about the loading of the heart and its interaction with homeostatic regulation. Insights acquired in the present opens the way for designing better optimization protocols, possibly even including computer modeling.

\section{Conclusion}

The LV and the RV respond in an opposite manner to LV or RV preexcitation. LV pre-excitation improved LV contractility and decreased RV contractility, while RV pre-excitation had the opposite effects. The CircAdapt computer model realistically captures these opposite responses of LV and RV contractile function. Computer simulations extend animal experimental findings by revealing that improving ventricular contractility does not necessarily lead to an improvement of cardiac output. This study demonstrates the potential of CircAdapt to provide a valuable and efficient in silico platform for further optimization studies for device therapy. 


\section{References}

Abraham, W. W. T., Fisher, W. G. W., Smith, A. L., Delurgio, D. B., Leon, A. R., Loh, E., et al. (2002). Cardiac resynchronization in chronic heart failure. N. Engl. J. Med. 346, 1845-53. doi:10.1056/NEJMoa013168.

Arts, T., Delhaas, T., Bovendeerd, P., Verbeek, X., and Prinzen, F. (2005). Adaptation to mechanical load determines shape and properties of heart and circulation: the CircAdapt model. Am. J. Physiol. Heart Circ. Physiol. 288, 1943-1954. doi:10.1152/ajpheart.00444.2004.

Arts, T., Lumens, J., Kroon, W., and Delhaas, T. (2012). Control of whole heart geometry by intramyocardial mechano-feedback: A model study. PLoS Comput. Biol. 8. doi:10.1371/journal.pcbi.1002369.

Arts, T., Prinzen, F. W., Snoeckx, L. H. E. H., Rijcken, J. M., and Reneman, R. S. (1994). Adaption of cardiac structure by mechanical feedback in the environment of the cell: A model study. Biophys. J. 66, 953-961. doi:10.1016/S0006-3495(94)808768.

Auger, D., Hoke, U., Bax, J. J., Boersma, E., and Delgado, V. (2013). Effect of atrioventricular and ventriculoventricular delay optimization on clinical and echocardiographic outcomes of patients treated with cardiac resynchronization therapy: A meta-analysis. Am. Heart J. 166, 20-29. doi:10.1016/j.ahj.2013.03.021.

Auricchio, A., and Prinzen, F. W. (2011). Non-Responders to Cardiac Resynchronization Therapy. Circ. J. 75, 521-527. doi:10.1253/circj.CJ-10-1268.

Bogaard, M. D., Meine, M., Tuinenburg, A. E., Maskara, B., Loh, P., and Doevendans, P. A. (2012). Cardiac resynchronization therapy beyond nominal settings: Who needs individual programming of the atrioventricular and interventricular delay? Europace 14, 1746-1753. doi:10.1093/europace/eus170.

Brignole, M., Auricchio, A., Baron-Esquivias, G., Bordachar, P., Boriani, G., Breithardt, O.A., et al. (2013). 2013 ESC Guidelines on cardiac pacing and cardiac resynchronization therapy: the Task Force on cardiac pacing and resynchronization therapy of the European Society of Cardiology (ESC). Developed in collaboration with the European Heart Rhythm Association . Eur. Heart J. 34, 2281-329. doi:10.1093/eurheartj/eht150.

Cleland, J. G., Daubert, J. C., Erdmann, E., Freemantle, N., Gras, D., Kappenberger, L., et al. (2005). The Effect of Cardiac Resynchronization on Morbidity and Mortality in Heart Failure. 352, 1539-1549. Available at: pm:15753115.

Cleveland, W. S. (1979). Robust locally weighted regression and smoothing scatterplots. J. Am. Stat. Assoc. 74, 829-836. doi:10.1080/01621459.1979.10481038.

Crozier, A., Blazevic, B., Lamata, P., Plank, G., Ginks, M., Duckett, S., et al. (2016). The relative role of patient physiology and device optimisation in cardiac resynchronisation therapy: A computational modelling study. J. Mol. Cell. Cardiol. 96, 93-100. doi:10.1016/j.yjmcc.2015.10.026. 
Groote, P. D. E., Millaire, A., Foucher-hossein, C., de Groote, P., Millaire, A., Foucherhossein, C., et al. (1998). Right ventricular ejection fraction is an independent predictor of survival in patients with moderate heart failure. J. Am. Coll. Cardiol. 32, 948-954. doi:http://dx.doi.org/10.1016/S0735-1097\%2898\%2900337-4.

Houston, B. A., Sturdivant, J. L., Yu, Y., and Gold, M. R. (2018). Acute biventricular hemodynamic effects of cardiac resynchronization therapy in right bundle branch block. Hear. Rhythm 15, 1525-1532. doi:10.1016/j.hrthm.2018.05.017.

Huntjens, P. R., Ploux, S., Strik, M., Walmsley, J., Ritter, P., Haissaguerre, M., et al. (2018). Electrical Substrates Driving Response to Cardiac Resynchronization Therapy. Circ. Arrhythmia Electrophysiol. 11, e005647. doi:10.1161/CIRCEP.117.005647.

Huntjens, P. R., Walmsley, J., Ploux, S., Bordachar, P., Prinzen, F. W., Delhaas, T., et al. (2014). Influence of left ventricular lead position relative to scar location on response to cardiac resynchronization therapy: a model study. Europace 16 Suppl 4, iv62-iv68. doi:10.1093/europace/euu231.

Hyde, E. R., Behar, J. M., Crozier, A., Claridge, S., Jackson, T., Sohal, M., et al. (2016). Improvement of Right Ventricular Hemodynamics With Left Ventricular Endocardial Pacing During Cardiac Resynchronization Therapy. Pacing Clin. Electrophysiol. 39, n/a-n/a. doi:10.1111/pace.12854.

Jones, S., Lumens, J., Sohaib, S. M. A., Finegold, J. A., Kanagaratnam, P., Tanner, M., et al. (2017). Cardiac resynchronization therapy: mechanisms of action and scope for further improvement in cardiac function. Europace 19, 1178-1186. doi:10.1093/europace/euw136.

Lee, A. W. C., Costa, C. M., Strocchi, M., Rinaldi, C. A., and Niederer, S. A. (2018). Computational Modeling for Cardiac Resynchronization Therapy. J. Cardiovasc. Transl. Res. 11, 92-108. doi:10.1007/s12265-017-9779-4.

Leenders, G. E., Lumens, J., Cramer, M. J., De Boeck, B. W. L., Doevendans, P. A., Delhaas, T., et al. (2012). Septal Deformation Patterns Delineate Mechanical Dyssynchrony and Regional Differences in Contractility: Analysis of Patient Data Using a Computer Model. Circ. Hear. Fail. 5, 87-96. doi:10.1161/CIRCHEARTFAILURE.111.962704.

Lumens, J., Blanchard, D. G., Arts, T., Mahmud, E., and Delhaas, T. (2010). Left ventricular underfilling and not septal bulging dominates abnormal left ventricular filling hemodynamics in chronic thromboembolic pulmonary hypertension. AJP Hear. Circ. Physiol. 299, H1083-H1091. doi:10.1152/ajpheart.00607.2010.

Lumens, J., Delhaas, T., Kirn, B., and Arts, T. (2009). Three-wall segment (TriSeg) model describing mechanics and hemodynamics of ventricular interaction. Ann. Biomed. Eng. 37, 2234-2255. doi:10.1007/s10439-009-9774-2.

Lumens, J., Ploux, S., Strik, M., Gorcsan, J., Cochet, H., Derval, N., et al. (2013). Comparative electromechanical and hemodynamic effects of left ventricular and biventricular pacing in dyssynchronous heart failure: Electrical resynchronization versus left-right ventricular interaction. J. Am. Coll. Cardiol. 62, 2395-2403. doi:10.1016/j.jacc.2013.08.715. 
Lumens, J., Tayal, B., Walmsley, J., Delgado-Montero, A., Huntjens, P. R., Schwartzman, D., et al. (2015). Differentiating Electromechanical from Non-Electrical Substrates of Mechanical Discoordination to Identify Responders to Cardiac Resynchronization Therapy. Circ. Cardiovasc. Imaging 8, 1-12. doi:10.1161/CIRCIMAGING.115.003744.

Miri, R., Graf, I. M., and DÖssel, O. (2009). Efficiency of timing delays and electrode positions in optimization of biventricular pacing: A simulation study. IEEE Trans. Biomed. Eng. 56, 2573-2582. doi:10.1109/TBME.2009.2027692.

Nguyên, U. C., Verzaal, N. J., van Nieuwenhoven, F. A., Vernooy, K., and Prinzen, F. W. (2018). Pathobiology of cardiac dyssynchrony and resynchronization therapy. EP Eur. 6, 1660-1665. doi:10.1093/europace/euy035.

Niederer, S., Mitchell, L., Smith, N., and Plank, G. (2011). Simulating human cardiac electrophysiology on clinical time-scales. Front. Physiol. APR. doi:10.3389/fphys.2011.00014.

Palau-Caballero, G., Walmsley, J., Van Empel, V., Lumens, J., and Delhaas, T. (2017). Why septal motion is a marker of right ventricular failure in pulmonary arterial hypertension: mechanistic analysis using a computer model. Am. J. Physiol. - Hear. Circ. Physiol. 312, H691-H700. doi:10.1152/ajpheart.00596.2016.

Peschar, M., Vernooy, K., Vanagt, W. Y. R., Reneman, R. S., Vos, M. A., and Prinzen, F. W. (2003). Absence of reverse electrical remodeling during regression of volume overload hypertrophy in canine ventricles. Cardiovasc. Res. 58, 510-517. doi:10.1016/S0008-6363(03)00331-6.

Ploux, S., Lumens, J., Whinnett, Z., Montaudon, M., Strom, M., Ramanathan, C., et al. (2013). Noninvasive electrocardiographic mapping to improve patient selection for cardiac resynchronization therapy: beyond QRS duration and left bundle branch block morphology. J. Am. Coll. Cardiol. 61, 2435-2443. doi:10.1016/j.jacc.2013.01.093.

Ploux, S., Strik, M., Van Hunnik, A., Van Middendorp, L., Kuiper, M., and Prinzen, F. W. (2014). Acute electrical and hemodynamic effects of multisite left ventricular pacing for cardiac resynchronization therapy in the dyssynchronous canine heart. Hear. Rhythm 11, 119-125. doi:10.1016/j.hrthm.2013.10.018.

Prinzen, F. W., Vernooy, K., and Auricchio, A. (2013). Cardiac resynchronization therapy: State-of-the-art of current applications, guidelines, ongoing trials, and areas of controversy. Circulation 128, 2407-2418. doi:10.1161/CIRCULATIONAHA.112.000112.

Quinn, T. A., Berberian, G., Cabreriza, S. E., Maskin, L. J., Weinberg, A. D., Holmes, J. W., et al. (2006). Effects of sequential biventricular pacing during acute right ventricular pressure overload. Am. J. Physiol. - Hear. Circ. Physiol. 291, H2380H2387. doi:10.1152/ajpheart.00446.2006.

Quinn, T. A., Cabreriza, S. E., Richmond, M. E., Weinberg, A. D., Holmes, J. W., and Spotnitz, H. M. (2009). Simultaneous variation of ventricular pacing site and timing with biventricular pacing in acute ventricular failure improves function by 
interventricular assist. Am. J. Physiol. Heart Circ. Physiol. 297, H2220-H2226. doi:10.1152/ajpheart.00802.2009.

Reumann, M., Farina, D., Miri, R., Lurz, S., Osswald, B., and Dössel, O. (2007). Computer model for the optimization of $\mathrm{AV}$ and $\mathrm{VV}$ delay in cardiac resynchronization therapy. Med. Biol. Eng. Comput. 45, 845-854. doi:10.1007/s11517-007-0230-x.

Ricci, F., Mele, D., Bianco, F., Bucciarelli, V., De Caterina, R., and Gallina, S. (2017). Right heart-pulmonary circulation unit and cardiac resynchronization therapy. Am. Heart J. 185, 1-16. doi:10.1016/j.ahj.2016.11.005.

Sciaraffia, E., Malmborg, H., Lönnerholm, S., Blomström, P., and Blomström Lundqvist, C. (2009). Right ventricular contractility as a measure of optimal interventricular pacing setting in cardiac resynchronization therapy. Europace 11, 1496-1500. doi:10.1093/europace/eup288.

Strik, M., Van Deursen, C. J. M., Van Middendorp, L. B., Van Hunnik, A., Kuiper, M., Auricchio, A., et al. (2013a). Transseptal conduction as an important determinant for cardiac resynchronization therapy, as revealed by extensive electrical mapping in the dyssynchronous canine heart. Circ. Arrhythmia Electrophysiol. 6, 682-689. doi:10.1161/CIRCEP.111.000028.

Strik, M., Van Middendorp, L. B., Houthuizen, P., Ploux, S., Van Hunnik, A., Kuiper, M., et al. (2013b). Interplay of electrical wavefronts as determinant of the response to cardiac resynchronization therapy in dyssynchronous canine hearts. Circ. Arrhythmia Electrophysiol. 6, 924-931. doi:10.1161/CIRCEP.113.000753.

Thibault, B., Ducharme, A., Harel, F., White, M., Omeara, E., Guertin, M. C., et al. (2011). Left ventricular versus simultaneous biventricular pacing in patients with heart failure and a qrs complex ???120 milliseconds. Circulation 124, 2874-2881. doi:10.1161/CIRCULATIONAHA.111.032904.

van Everdingen, W. M., Zweerink, A., Salden, O. A. E., Cramer, M. J., Doevendans, P. A., van Rossum, A. C., et al. (2018). Atrioventricular optimization in cardiac resynchronization therapy with quadripolar leads: should we optimize every pacing configuration including multi-point pacing? EP Eur., 1-9. doi:10.1093/europace/euy138.

Vernooy, K., Cornelussen, R. N. M., Verbeek, X. A. A. M., Vanagt, W. Y. R., Van Hunnik, A., Kuiper, M., et al. (2007). Cardiac resynchronization therapy cures dyssynchronopathy in canine left bundle-branch block hearts. Eur. Heart J. 28, 2148-2155. doi:10.1093/eurheartj/ehm207.

Walmsley, J., Arts, T., Derval, N., Bordachar, P., Cochet, H., Ploux, S., et al. (2015a). Fast Simulation of Mechanical Heterogeneity in the Electrically Asynchronous Heart Using the MultiPatch Module. PLoS Comput. Biol. 11, e1004284. doi:10.1371/journal.pcbi.1004284.

Walmsley, J., Huntjens, P. R., Prinzen, F. W., Delhaas, T., and Lumens, J. (2015b). Septal flash and septal rebound stretch have different underlying mechanisms. Am. J. Physiol. Heart Circ. Physiol., ajpheart.00639.2015. doi:10.1152/ajpheart.00639.2015. 



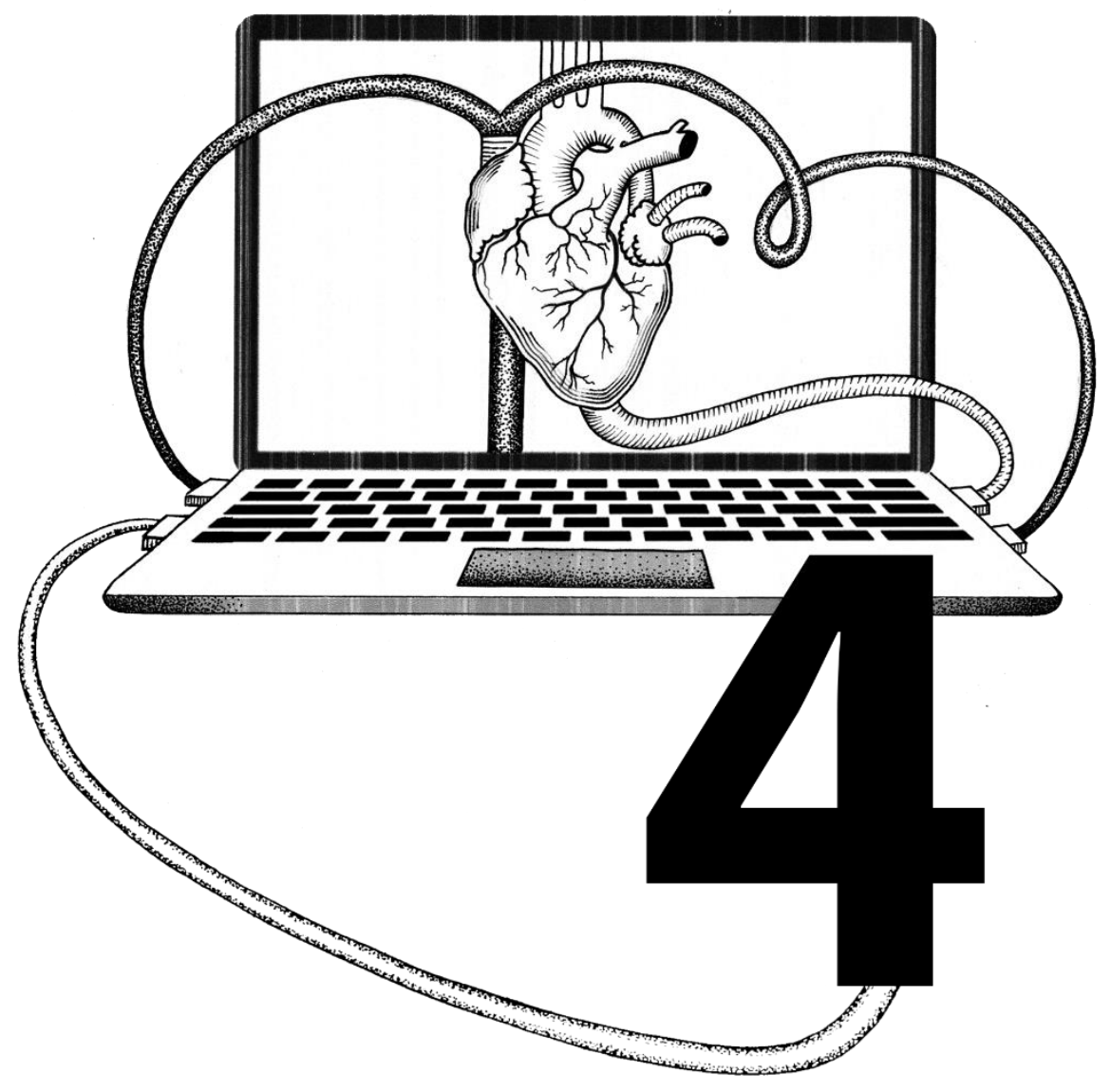




\section{Chapter 4}

\section{Impact of paced left}

ventricular dyssynchrony on left ventricular reverse remodeling after cardiac resynchronization therapy

Anaïs Gauthey*, Erik Willemen, Joost Lumens, Sylvain Ploux, Pierre Bordachar, Philippe Ritter, Frits W. Prinzen, Sibille Lejeune, Anne-Catherine Pouleur, Quentin Garnir, Sébastien Marchandise, Christophe Scavée, Aurélien Wauters, JeanBenoit le Polain de Waroux

*Anaïs Gauthey and Erik Willemen contributed equally to this work.

J Cardiovasc Electrophysiol. 2020;1-9. doi:10.1111/jce.14330 


\section{Abstract}

Introduction: We investigated whether pacing-induced electrical dyssynchrony at the time of cardiac resynchronization therapy (CRT) device implantation was associated with chronic CRT response.

Methods and Results: We included a total of 69 consecutive heart failure patients who received a CRT device. Left (LVp-RVs) and right ( $R \bigvee p$-LVs) pacing-induced interlead delays were measured intraoperatively and used to determine if there was paced left ventricular (LV) dyssynchrony, defined as present when LVp-RVs is larger than RVp-LVs. CRT response was defined as a reduction in LV end-systolic volume $\geq 15 \%, 6$ months after implantation. Paced left ventricular dyssynchrony (PLVD) was associated with ischemic cardiomyopathy (ICM) ( $\chi 2: 8 ; P=.005)$ but not with QRS morphology nor with pacing lead positions. In a univariate analysis, PLVD (odds ratio [OR], 6.53; 95\% confidence interval [Cl], 2.2-18.9; $\mathrm{P}=.001)$, atypical left bundle branch block (LBBB) $(\mathrm{OR}, 3.3 ; 95 \% \mathrm{Cl}$, 1.2-9.4; $\mathrm{P}=.022)$, and ICM $(\mathrm{OR}, 5.2 ; 95 \% \mathrm{Cl}, 1.6-17 ; \mathrm{P}=.006)$ were associated with nonresponse. In a multivariate analysis, both PLVD $(\mathrm{OR}, 9.74 ; 95 \% \mathrm{Cl}, 2.8-33.9 ; \mathrm{P}<.0001)$ and atypical LBBB (OR, 5.6; $95 \% \mathrm{Cl}, 1.5-20.3 ; \mathrm{P}=.009$ ) were independently associated with nonresponse. Adding PLVD to a model based on QRS morphology provided a significant and meaningful incremental value to predict LV reverse remodeling after CRT ( $\chi 2$ to enter: 8; $P<.005$ ).

Computer simulations corroborate these findings by showing that, while intrinsic electrical dyssynchrony is a prerequisite, the level of pacing-induced dyssynchrony modulates acute CRT response.

Conclusion: In addition to the intrinsic electrical substrate, PLVD is strongly associated with less LV reverse remodeling, demonstrating that measuring the electrical substrate during pacing has additional value for prediction of CRT response in an already well-selected patient population. 


\section{Introduction}

Cardiac resynchronization therapy (CRT) reduces morbimortality and improves heart failure symptoms in appropriately selected candidates ${ }^{1}$. The likelihood of nonresponse to CRT increases with ischemic cardiomyopathy (ICM), atypical left bundle branch block (LBBB), narrow QRS complex, and less activation delay at the site of left ventricular (LV) lead implant (QLV) ${ }^{2-4}$. Despite improvements in guiding LV lead implantation using both electrical and imaging strategies ${ }^{5-7}$, the rate of nonresponse to CRT remains around onethird of the patients. In this context, underlying electrical patterns of nonresponse have been poorly described and electrocardiographic criteria (QRS duration and morphology) still remain insufficient to differentiate responders from nonresponders to CRT.

The working mechanism of CRT consists of restoring electromechanical ventricular synchrony. By definition, the potential for CRT response lies in the baseline electrical substrate inherent to the patient, but also in the electromechanical effect of biventricular pacing. While most attempts to predict CRT response have focused on the patient's baseline electromechanical substrate, the role of pacing-induced electrical dyssynchrony has been poorly studied ${ }^{8}$. However, understanding the interplay between baseline and pacing-induced dyssynchrony might be important to better predict nonresponse to CRT. Theoretically, one would expect the least pacing-induced left ventricular dyssynchrony (PLVD) and most homogeneous pacing-induced wavefront propagation when the right ventricular paced to left ventricular sensed delay (RVp-LVs) is similar to the left ventricular paced to right ventricular sensed delay (LVp-RVs). By contrast, asymmetrical paced-to-sensed delays may indicate a more heterogeneous ventricular activation compromising the potential benefits of CRT. 


\section{0 | Chapter 4}

In the present study, we explored prospectively whether interlead paced-to-sensed delays recorded at the time of implant could predict a positive response to CRT. More precisely, we hypothesized that larger LV dyssynchrony during pacing can predict nonresponse. In addition, the relative contributions of baseline dyssynchrony and interlead paced-to-sensed delays to CRT response are studied in a more well-controlled in silico environment, using the well-validated CircAdapt model ${ }^{9-11}$ of the human heart and cardiovascular system.

\section{Methods}

\section{Population and study protocol}

The study population consisted of 69 consecutive heart failure patients who received a CRT device based on current guideline indications ${ }^{1}$. Only patients with New York Heart Association Functional Classification classes II-III or IV despite optimal medical therapy, left ventricular ejection fraction (LVEF) $\leq 35 \%$, and QRS duration $\geq 130$ ms were included. CRT response was defined as a reduction in LV end-systolic volume $\geq 15 \%{ }^{12}, 6$ months after implant compared to baseline. Patients with a previous implantable cardiac electronic device, below the age of 18, congenital disease, and pregnant women were excluded from inclusion. Focal and diffuse fibrosis were assessed on cardiac magnetic resonance imaging (cMRI) when available before implantation. Typical LBBB electrocardiogram (ECG) criteria were based on Strauss and ESC 2013 classifications ${ }^{13}$. The study was approved by our local ethics committee and all patients consented to participate in this study. 


\section{CRT implantation}

Transvenous CRT implantation was performed according to standard procedures. Right ventricular (RV) lead was positioned at the RV apex or in a midseptal position. Guided LV lead implantation was performed using coronary sinus venogram and the latest site of activation represented by QLV measurement was reached. The final LV lead position was confirmed if pacing thresholds were acceptable with a good LV lead stability and after excluding phrenic nerve stimulation. LV lead location was documented using anteroposterior, left and right anterior oblique fluoroscopic views, and defined as anterior, lateral or posterior, and apical, mid, or basal. Simultaneous biventricular stimulation was programmed and the atrioventricular delay was chosen to avoid A-wave truncation.

\section{Intraoperative electrical delay assessment}

Electrical sensed and paced delays between the distal left bipole and the RV leads, as well as the QLV, were recorded using the device programmer (Abbott Merlin Pacing System Analyzer Model EX3100) at a sweep speed of $100 \mathrm{~mm} / \mathrm{s}$ (Figure 1). QLV was assessed by aligning the calipers with the onset of the QRS and the peak of the LV electrogram. RVs-LVs delays (ms) were measured between the peak in the RV electrogram and the peak in the LV electrogram during spontaneous rhythm. Paced interlead delays were defined as the conduction time ( $\mathrm{ms}$ ) from the LVp artifact to $R V$ s electrogram and from the RVp artifact to LVs electrogram. Pacing was performed at the minimum rate that ensured complete ventricular capture (excluding fusion beats). Bipolar configuration was programmed for both pacing and sensing recordings. A standard pacing output of 5 V/0.4 ms was applied. PLVD was defined as a situation where LVp-RVs is greater than RVp-LVs delays, indicating a more dyssynchronous activation of the LV 


\section{2 | Chapter 4}

during pacing. All delays were carefully reviewed and confirmed in all except one patient. Whenever possible (absence of phrenic nerve stimulation or inacceptable thresholds), measurements were performed from the distal bipole. In any cases, the initial LV configuration programming was the same as the one tested during the implant procedure.
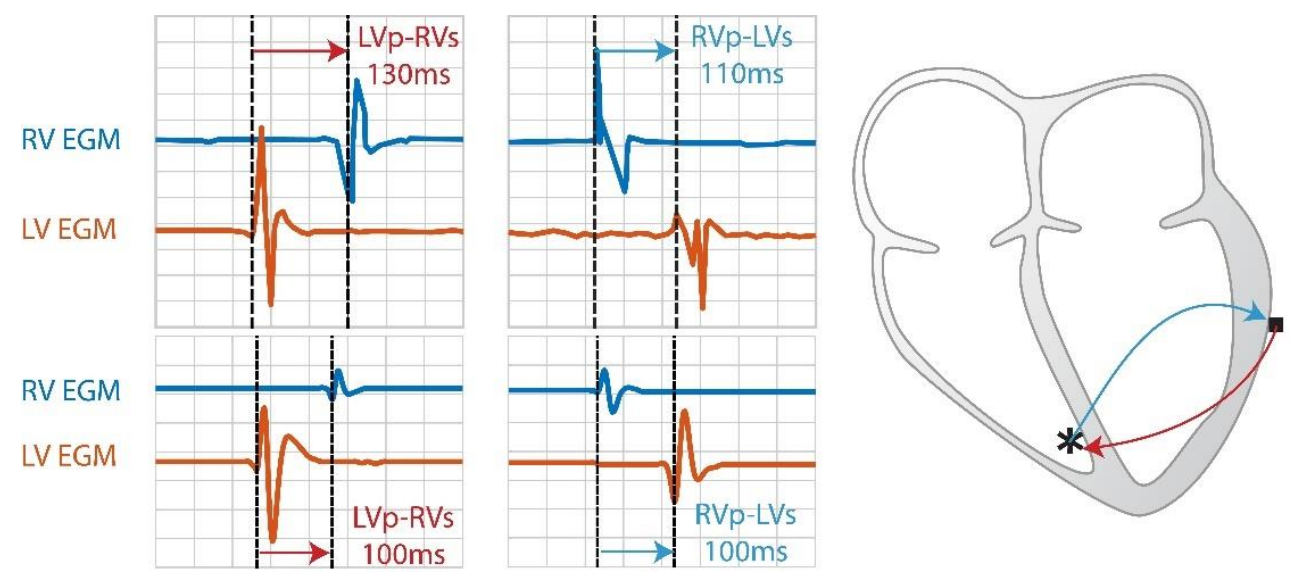

Figure 1 Intraoperatively measured RV (top) and LV (bottom) electrogram of RVp-LVs (blue arrow) and LVp-RVs (red arrow). Top shows an example of paced left ventricular dyssynchrony since $L V p-R V s>R V p-L V s$. Bottom shows an example of synchronous activation. LV-EGM, left ventricular electrogram; $L V p-R V s$, interval (ms) from left ventricular pacing to right ventricular sensed event on electrograms; PLVD, paced left ventricular dyssynchrony with ( LVp-RVs) - (RVp-LVs) > ; RV-EGM, right ventricular electrogram; $R V p-L V s$, interval (ms) from right ventricular pacing to left ventricular sensed event on electrograms 


\section{Echocardiography}

Transthoracic echocardiography using an iE33 and an Epic ultrasound system (Philips Medical Systems; Andover, MA) with a 1 - to 5 - $\mathrm{Hz}$ transthoracic transducer was performed at baseline and after a minimum of 6 months (range: 6-12 months) postimplant. LV volumes and LVEF were obtained using the biplane Simpson's method from four and two chambers' views. Measurements were obtained using the Philips IntelliSpace Cardiovascular Software (version 2.2). Offline analyses were performed by two independent observers ( $\mathrm{AG}$ and $\mathrm{SL}$ ) and intraclass correlation coefficient between both was very good $(0.86 ; 95 \% \mathrm{Cl}, 0.56-0.96)$.

\section{Statistical Analysis}

Continuous variables are reported as mean \pm standard deviation or median and interquartile range. A comparison between groups was performed using independent t test when normally distributed or using the Kruskal-Wallis test if not. Categorical variables, expressed as counts and percentages, were compared using Fisher's exact test. All available clinical and electrocardiographic data (including all variables already identified in the literature as influencing the rate of response to CRT (age, sex, type of cardiomyopathy, LVEF, QRS duration, type of block, etc) were first used in a univariate regression analysis. All variables that correlated significantly $(P<$ .05) with the outcome (LVESV, $<-15 \%$ ) were then entered into a multivariate logistic regression. The model was then evaluated using the $\chi 2$ to enter. $P<.05$ was considered as significant.

Statistical analyses were done using IBM SPSS statistics software, version 25.0 . 


\section{4 | Chapter 4}

\section{Computer Simulations}

The CircAdapt computer model of the heart and circulation ${ }^{14}$ (MultiPatch Version) was used to evaluate the effect of baseline and paced dyssynchrony on acute hemodynamic response to pacing within the range measured in the patient population. Previous experimental and clinical studies have shown that the CircAdapt model realistically relates local ventricular myofiber mechanics to hemodynamics in the dyssynchronous failing heart?

In the simulations, we tested different levels of PLVD by inducing latency at the LV pacing site. Latency is defined as the time difference between the LV stimulus and the actual myocardial capture. From previous research, it is known that latency is often longer at the epicardial LV pacing site compared to the endocardial $\mathrm{RV}$ pacing site $^{15}$. In the simulations, latency is modeled as an additional delay between LV stimulus and the regional onset of mechanical activation.

Starting from a failing heart reference simulation, the LV total activation time was varied between 100 and $180 \mathrm{~ms}$ in steps of $10 \mathrm{~ms}$, resulting in nine baseline simulations. For each degree of baseline dyssynchrony, LV latencies from 0 to $50 \mathrm{~ms}$ (in steps of $5 \mathrm{~ms}$ ) were tested. The acute hemodynamic response was determined by calculating the change in maximum range in LV

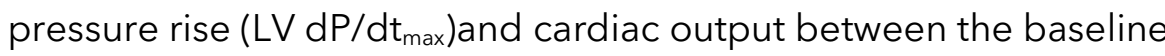
and pacing simulations. 


\section{Results}

\section{Patient population}

Baseline characteristics of this study population, summarized in Table 1, are similar to other published CRT cohorts. Responders and nonrespon- ders patients differed in terms of rate of nonischemic cardiomyopathy (NICM), typical LBBB, and QLV. Also,PLVD was present insignificantly more nonresponders compared to responders $(P<.0001)$. More than two-thirds of nonresponders presented with PLVD. On a quantitative analysis, compared to baseline, change in left ventricular end-systolic volume was significantly lower with PLVD compared to no PLVD (Figure 2).

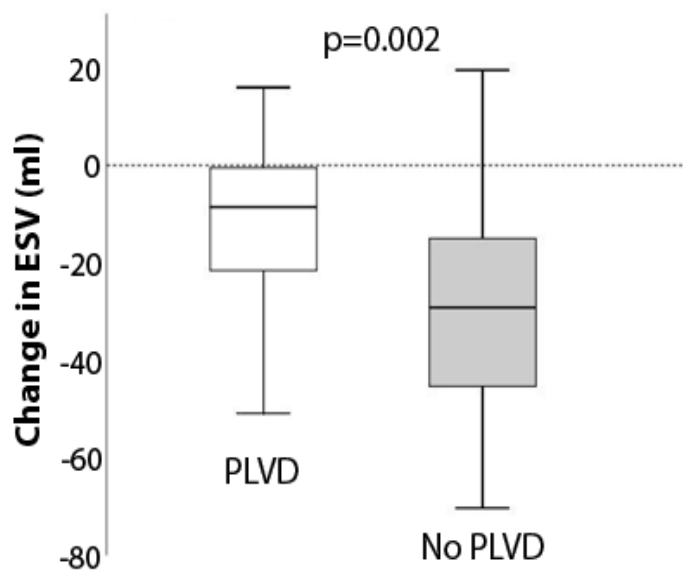

Figure 2 - Comparison of the change in LVESV $(\mathrm{mL})$ from baseline to 1-year postimplantation for the two patterns of delays (PLVD and no PLVD; ms). ESV, end-systolic volume; LVESV, left ventricular end-systolic volume $(m L)$; No $P L V D$, no paced left ventricular dyssynchrony with ( $L V p-R V s)$ $(R V p-L V s) \leq 0$ 


\section{Intraoperative LV to RV interlead delays and CRT response}

As indicated in Table 2, intrinsic interlead electrical sensed delay (RVs-LVs) was not different between groups, whereas PLVD occurred more often in nonresponders. Because of atrioventricular block, no RVs-LVs measurements were available in 7 of the 69 patients.

In the nonresponse group, the pace-to-sense time was $15 \mathrm{~ms}$ longer for the LV compared to the RV. PLVD was associated with ICM ( $P=.005)$ but not with the type of LBBB (typical vs atypical), sex, QRS duration, nor LV and RV lead positions. In $20 \%$ of the patients, PLVD resulted from a shorter conduction time from the right during $\mathrm{RV}$ pacing compared to the intrinsic wavefront propagation ( $R V p L V s<R V s L V s$ ). A comparison of patient's characteristics with PLVD and those without is described in Table 3.

With univariate analysis, PLVD $(\mathrm{OR}, 6.5 ; 95 \% \mathrm{Cl}, 2.2-18.9 ; \mathrm{P}=.001)$, atypical LBBB (OR, 3.3; 95\% Cl, 1.2-9.4; $\mathrm{P}=.022)$, and ICM (OR, 5.2; $95 \% \mathrm{Cl}, 1.6-17 ; \mathrm{P}=.006)$ were predictors for $\mathrm{CRT}$ nonresponse.

With multivariate analysis, both PLVD (OR, 9.7; 95\% Cl, 2.8-33.9; $\mathrm{P}<$ $.0001)$ and atypical LBBB (OR, 5.6; 95\% Cl, 1.5-20.3; $\mathrm{P}=.009)$ were independent predictors of nonresponse (Table 4). Accordingly, we developed an integrated statistical model that included synchronous activation of the LV during pacing (no PLVD) and the QRS morphology (typical LBBB). Our results show that compared to typical LBBB, only (which is the "first-line" screening tool for clinicians to predict CRT response), a model integrating both no PLVD+ typical LBBB had a better predictive value for CRT response ( $x 2$ to enter: $7.96 ; P<.005)$. 
Table 1: Comparison of baseline characteristics between responders and non-responders to CRT.

\begin{tabular}{|c|c|c|c|c|}
\hline & $\begin{array}{c}\text { Total } \\
\text { cohort } \\
(N=69)\end{array}$ & $\begin{array}{l}\text { Responders } \\
(\mathbf{N}=39)\end{array}$ & $\begin{array}{c}\text { Non- } \\
\text { responders } \\
(\mathbf{N}=\mathbf{3 0})\end{array}$ & $\begin{array}{r}\text { P. } \\
\text { value* }\end{array}$ \\
\hline$\overline{\operatorname{Age}(Y)}$ & $66(21)$ & $65(21)$ & $67(18)$ & 0.2 \\
\hline $\begin{array}{l}\text { Gender (males, } \\
\% \text { ) }\end{array}$ & 70 & 61 & 80 & 0.1 \\
\hline $\operatorname{NICM}(\%)$ & 74 & 87 & 57 & 0.004 \\
\hline NYHA II- III (\%) & 85 & 87 & 83 & 0.7 \\
\hline $\begin{array}{l}\text { Typical LBBB } \\
(\%)\end{array}$ & 65 & 77 & 50 & 0.02 \\
\hline $\begin{array}{l}\text { QRS duration } \\
\text { (ms) }\end{array}$ & $160(20)$ & $160(20)$ & $160(20)$ & 0.8 \\
\hline LVEF (\%) & $25 \pm 6$ & $24 \pm 6$ & $23 \pm 6$ & 0.9 \\
\hline $\operatorname{LVEDV~}\left(\mathrm{mL} / \mathrm{m}^{2}\right)$ & $\begin{array}{r}250 \\
(134)\end{array}$ & 250 (139) & 248 (129) & 0.4 \\
\hline $\begin{array}{l}\text { LV pacing site } \\
\text { Lateral or } \\
\text { posterolateral } \\
\text { (\%) }\end{array}$ & 92.6 & 92 & 93 & 0.8 \\
\hline $\begin{array}{l}\text { RV lead } \\
\text { position (\%) } \\
\text { Midseptal } \\
\text { Apical }\end{array}$ & $\begin{array}{l}25 \\
75\end{array}$ & $\begin{array}{l}26 \\
74\end{array}$ & $\begin{array}{l}23 \\
77\end{array}$ & 0.8 \\
\hline QLV (ms) & $132 \pm 46$ & $150 \pm 50$ & $113 \pm 33$ & 0.03 \\
\hline $\begin{array}{l}\text { QLV ratio ( } \geq 50 \\
\%)\end{array}$ & 89 & $87(13 / 15)$ & $92(12 / 13)$ & 0.6 \\
\hline PLVD & $\begin{array}{r}46 \% \\
(N=31)\end{array}$ & $26 \%(N=10)$ & $70 \%(N=21)$ & $<0.0001$ \\
\hline
\end{tabular}

Note: Values are mean $\pm S D$ or median (IQR).

Abbreviations: CRT, cardiac resynchronization therapy; ECG, electrocardiogram; IQR, interquartile range; $L B B B$, left bundle branch block; LV, left ventricular; LVEDV, left ventricular end-diastolic volume; $L V E F$, left ventricular ejection fraction; NICM, nonischemic cardiomyopathy; NYHA, New York Heart Association; PLVD, paced left ventricular dyssynchrony; $Q L V$, interval ( $m s$ ) from the onset of the $Q R S$ on the surface ECG to the first peak of left ventricular electrogram during sinus rhythm; $R V$, right ventricular. ${ }^{\star} P<.05$ (Kruskal-Wallis test, independent $t$ test, or $\chi 2$ test). 
Table 2 - Comparison of LV-RV delays at the time of implant between responders and nonresponders

\begin{tabular}{lrrrr}
\hline & $\begin{array}{r}\text { Total } \\
\text { cohort } \\
(\mathbf{N =} 68)\end{array}$ & $\begin{array}{r}\text { Responders } \\
\mathbf{( N = 3 9 )}\end{array}$ & $\begin{array}{r}\text { Nonresponders } \\
\mathbf{( N = 3 0 )}\end{array}$ & P-value* $^{*}$ \\
\hline RVs-LVs & $82 \pm 44$ & $89 \pm 45$ & $72 \pm 40$ & 0.13 \\
LVp-RVs & $120 \pm 41$ & $116 \pm 43$ & $126 \pm 38$ & 0.35 \\
RVp-LVs & $115 \pm 37$ & $120 \pm 40$ & $111 \pm 34$ & 0.43 \\
$(L V p-R V s)-$ & $5 \pm 23$ & $-2 \pm 21$ & $14 \pm 23$ & 0.003 \\
$(R V p-L V s)$ & & & $70 \%$ & $<0.0001$ \\
\hline PLVD & $46 \%$ & $26 \%$ & & \\
\hline
\end{tabular}

Note: Values are mean $\pm S D$.

Abbreviations: $L V$, left ventricular; $L V p-R V s$, interval (ms) from left ventricular pacing to right ventricular sensed event; $P L V D$, paced left ventricular dyssynchrony; $R V$, right ventricular; $R V p-L V s$, interval (ms) from right ventricular pacing to left ventricular sensed event; $R V s-L V s$, interval ( $m s$ ) from right to left ventricular first peak event on electrograms in sinus rhythm.

${ }^{\star} P<.005$ (independent $t$ test or $\chi 2$ test).

Table 3 - Comparison of characteristics between patients with and without paced left ventricular dyssynchrony

\begin{tabular}{lrrr}
\hline & $\begin{array}{r}\text { PLVD } \\
(\mathbf{N}=31)\end{array}$ & $\begin{array}{r}\text { No PLVD } \\
(\mathbf{N = 3 7 )}\end{array}$ & P value* $^{*}$ \\
\hline Age (Y) & $65(18)$ & $66(20)$ & 0.34 \\
Gender (males, \%) & 74 & 67 & 0.5 \\
NICM (\%) & 61 & 84 & 0.04 \\
NYHA II- III (\%) & 87 & 80 & 0.7 \\
Typical LBBB (\%) & 68 & 62 & 0.6 \\
QRS duration (ms) & $160(20)$ & $160(20)$ & 0.8 \\
LVEF (\%) & $25 \pm 6$ & $24 \pm 6$ & 0.7 \\
LVEDV (mL/m²) & $260(140)$ & $243(129)$ & .7 \\
LV pacing site & & & 1 \\
$\quad$ Lateral or posterolateral (\%) & 92 & 93 & \\
RV lead position (\%) & & & 0.1 \\
$\quad$ Midseptal & 32 & 17 & \\
\hline
\end{tabular}




\begin{tabular}{lrrr}
\hline \multicolumn{1}{c}{ Apical } & 68 & 83 & \\
$\mathrm{QLV}(\mathrm{ms})$ & $122 \pm 47$ & $138 \pm 47$ & 0.4 \\
$\mathrm{QLV}$ ratio $(\geq 50 \%)$ & 85 & 93 & 0.5 \\
$\Delta \mathrm{ESV} \geq 15 \%(\%)$ & 32 & 76 & $<0.0001$ \\
\hline & $\begin{array}{r}\text { PLVD } \\
(\mathbf{N}=\mathbf{3 1})\end{array}$ & $\begin{array}{r}\text { No PLVD } \\
(\mathbf{N}=\mathbf{3 7})\end{array}$ & P value* $^{*}$ \\
\hline
\end{tabular}

Note: Values are mean \pm SD.

Abbreviations: ESV, end-systolic volume; IQR, interquartile range; LBBB, left bundle branch block; LGE, late gadolinium enhancement; LV, left ventricular; LVEDV, left ventricular end-diastolic volume; LVEF, left ventricular ejection fraction; NICM, nonischemic cardiomyopathy; NYHA, New York Heart Association; PLVD, paced left ventricular dyssynchrony; $\mathrm{RV}$, right ventricular.

${ }^{\star} \mathrm{P}<.05$ (independent $\mathrm{t}$ test, Kruskal-Wallis or $\mathrm{x} 2$ tests).

Table 4 - Predictors for CRT non-response at univariate and multivariate logistic regression analyses.

\begin{tabular}{|c|c|c|c|c|}
\hline \multicolumn{3}{|c|}{ Univariate analysis } & \multicolumn{2}{|c|}{ Multivariate analysis } \\
\hline & $\begin{array}{r}\text { Odds ratio } \\
(95 \% \mathrm{Cl})\end{array}$ & P-value & $\begin{array}{r}\text { Odds } \\
\text { ratio } \\
(95 \% \mathrm{Cl})\end{array}$ & P-value \\
\hline Gender (male) & $2.6(0.86-7.88)$ & $P=0.089$ & & \\
\hline Age & $1.027(0.98-1.07)$ & $P=0.19$ & & \\
\hline Baseline LEVF & $0.98(0.91-1.06)$ & $P=0.76$ & & \\
\hline QRS duration & 0.99 (0.97-1.017) & $P=0.58$ & & \\
\hline QLV ratio $\geq 50 \%$ & $0.54(0.04-6.76)$ & $P=0.63$ & & \\
\hline PLVD & $6.53(2.25-18.92)$ & $P=0.001$ & $\begin{array}{r}9.74(2.8- \\
33.9)\end{array}$ & $<0.0001$ \\
\hline Atypical LBBB & $3.33(1.19-9.36)$ & $P=0.022$ & $\begin{array}{r}5.6(1.5- \\
20.3)\end{array}$ & 0.009 \\
\hline ICM & $5.2(1.6-17)$ & $P=0.006$ & & \\
\hline
\end{tabular}

Abbreviations: $\mathrm{Cl}$, confidence interval; $C R T$, cardiac resynchronization therapy; ICM, ischemic cardiomyopathy; $\angle B B B$, left bundle branch block; $\angle V E F$, left ventricular ejection fraction; PLVD, paced left ventricular dyssynchrony 


\section{Relationship between interstitial fibrosis and necrosis and asymmetrical delays of conduction Interstitial}

Interstitial fibrosis and necrosis in heart failure are common substrates associated with localized conduction abnormalities, including unidirectional slowing and block of conduction potentially explaining our findings ${ }^{16,17}$. Accordingly, using CMRI, we investigated in 62 patients of our cohort whether PLVD was associated with a higher volume of focal (late gadolinium enhancement [LGE]) and diffuse fibrosis (extracellular volume [ECV]). Transmural LGE was present more often in patients with vs without PLVD (OR, 7.6; 95\% Cl, 1.5-38.8; $\mathrm{P}=.01$ ). Although there was a trend for higher ECV in nonresponders, ECV was not associated with PLVD (OR, 0.9; 95\% $\mathrm{Cl}, 0.9-1 ; \mathrm{P}=\mathrm{NS})$.

\section{Simulated acute hemodynamic responses evaluation of the interplay of baseline dyssynchrony and interlead paced delays}

Simulated acute hemodynamic responses to different baseline and pacing-induced dyssynchronies are presented in Figures 3 and 4. Both LV dP/dt $t_{\max }$ and cardiac output had an increased response to CRT when introducing a larger degree of baseline dyssynchrony. For a given degree of baseline dyssynchrony, the maximal response was observed when no LV latency was introduced in the model. Increasing the LV latency was associated with deterioration of both parameters, which could be especially important in situations where baseline dyssynchrony was lower. 

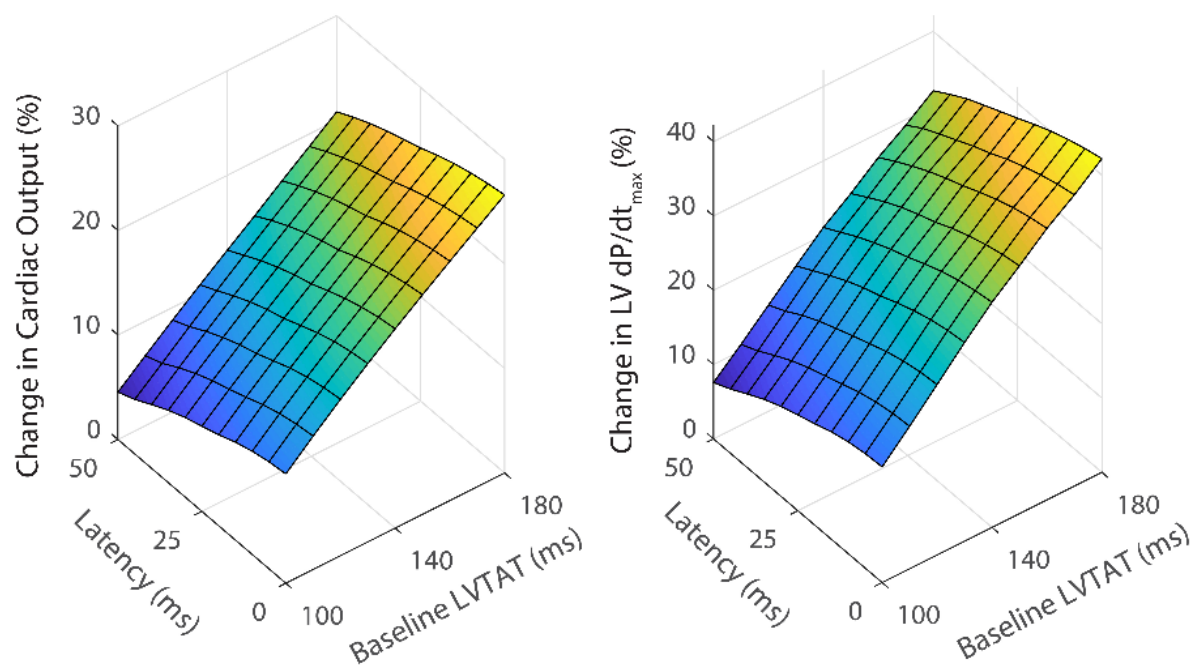

Figure 3 - Surface plots of the simulated acute changes in Cardiac Output (left) and $L V d P / d t_{\max }$ (right) when going from baseline dyssynchrony to CRT pacing with variation of levels of baseline dyssynchrony ( left ventricular total activation time (LVTAT): $100-180 \mathrm{~ms}$ ) and amount of latency at the LV pacing site (0-50ms).
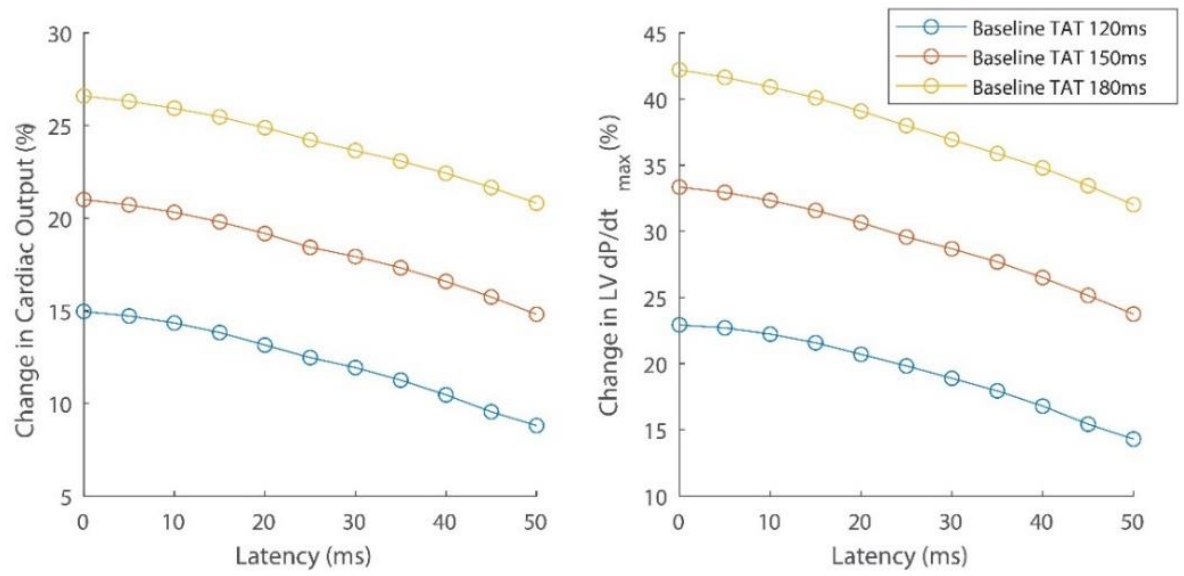

Figure 4 - Simulated acute changes in cardiac output (left) and $L V d P / d t_{\max }$ (right) when going from baseline dyssynchrony (left ventricular total activation time) to CRT pacing with different latencies at the LV pacing site (0-50 ms) during pacing. CRT, cardiac resynchronization therapy; $L V$, left ventricular; TAT, turnaround time 


\section{Discussion}

In the present study, we explored prospectively whether pacinginduced electrical activation delays recorded at the time of implant could predict CRT response. More precisely, we hypothesized that asymmetrical conduction times when pacing alternatively from the $L V$ and the RV leads can be used to predict LV reverse remodeling after CRT.

Our results can be summarized as follows:

- First, PLVD was strongly associated with the absence of LV reverse remodeling after CRT.

- Second, longer QLV values were associated with positive LV reverse remodeling. Where the presence of sufficient baseline dyssynchrony remains essential for a potential response, adding PLVD to a conventional statistical model based on QRS duration and morphology, significantly improved its predictive value.

- Third, computer simulations based on the range of dyssynchrony measured in the patient cohort confirmed that the level of pacing- induced electrical dyssynchrony modulates CRT response together with the baseline dyssynchrony. 


\section{PLVD better predicts CRT response because it incorporates the state of dyssynchrony during treatment}

A slower conduction velocity from the left is associated with nonresponse due to a decreased effectiveness of the resynchronization therapy. If pacing from the left to the right takes longer compared to the opposite, this results in a longer mean LV and total activation times, less synchronous activation, and acute response. From a clinical perspective, previous clinical studies already demonstrated that biventricular pacing provide CRT response mainly by reducing the interventricular dyssynchrony. Ploux et al ${ }^{18}$ demonstrated that effective biventricular pacing depends mainly on the reduction of ventricular electrical uncoupling (VEU). This electrical marker of dyssynchrony corresponds to the difference between the mean LV activation time minus the mean RV activation time during baseline and biventricular pacing. The more VEU is reduced the more hemodynamics acute benefit was observed with CRT. Our data not only confirm these results by pointing out the relative importance of both the intrinsic- and pacing-induced dyssynchrony but also demonstrate their implications for reverse remodeling and longterm clinical response. Hemodynamically, the acute increase in the cardiac output indicates that for the same cardiac output a lower preload is sufficient, lowering finally both the end-diastolic and end-systolic volumes. 


\section{Anisotropic conduction properties as a cause of nonresponse to CRT}

From a pathophysiological point of view, anisotropic conduction properties in the heart are not uncommon. Epicardial pacing sites are associated with slurred initiation of the ventricular activation. And, in some circumstances, the initiation of the activation front might be retained from the pacing site. Previous studies ${ }^{19-21}$ demonstrated that changing pacing sites might create an intermittent line of functional block and reroute the depolarization front. This has been reported as a possible cause for prolonged activation times and nonresponse. In the present study, we confirmed using computer simulations that an increase in latency at the LV pacing site results in a decrease in acute hemodynamic response.

Transmural LGE and history of ICM were present more often in both the PLVD and CRT nonresponse group. Differences in activation alone might thus not be the only reason for the association of PLVD with nonresponse. It appears this intraoperatively obtained conduction difference could potentially be an indicator for the level of myocardial tissue damage. It has previously been shown in multiple studies that LV reverse structural remodeling occurs less in ICM vs patients with NICM receiving $\mathrm{CRT}^{22}$.

\section{Markers of intrinsic dyssynchrony in a population selected on guideline criteria}

By exploring the interplay between both the baseline dyssynchrony and the pacing-induced dyssynchrony, the difference of interlead delays unravels electrophysiological characteristics that are otherwise not appreciated by our routine practice. The results of 
this study also highlight that it is hard to find an improved measure for intrinsic dyssynchrony in a population that is selected based on the current CRT guidelines. In contrast to other studies ${ }^{23-25}$, in our study population, neither the RVs-LVs nor QLV ratio ( $\geq 50 \%$ ) was significantly different between responders and nonresponders. Only the absolute QLV and typical LBBB differed significantly between both groups. The mean QLV also tended to be longer in patients without PLVD. Accordingly, results of the simulations indicated that a patient without PLVD might, therefore, show a larger acute response to pacing because this group has a relatively larger intrinsic dyssynchrony at baseline but also less paced dyssynchrony during biventricular pacing.

\section{Clinical implications}

Predicting CRT response is essential for correctly choosing between CRT-P and CRT-D ${ }^{1}$. Therefore, electrical measures using the pacing leads during implant might be important for improving the selection process of the device. Also, a better predictive model might be important for device optimization. Although not tested in the present study, patients with PLVD might benefit from LV preexcitation, either through setting a ventriculoventricular pacing delay or LV-only pacing with optimal fusion. Additional studies are warranted to check if LV pre-excitation truly improve CRT response rate in patients with PLVD.

\section{Limitations}

This study has some limitations worth noting. First, values of intraoperative delays are limited by the precision of measurements made with the programmer nonetheless, in the present study, all the recording delays were measured not from the ventricular markers but on the local electrogram (sensed or paced). This 


\section{6 | Chapter 4}

contributed to reducing a potential bias induced by a possible delay between the marker and the onset of the ventricular event. Second, all patients had the RV lead implanted at midseptal or apical position, irrespective of the presence of an identified scar. However, paced-to-sensed interlead delay was not associated to RV or LV lead position in the patients. Measuring the latency based on a 12-lead ECG would also have been of interest. When defining endpoints and choosing criteria to define CRT response, we decided to use LV remodeling and end-systolic volume only. We did not evaluate the clinical response to CRT and did not perform functional tests. Therefore, we cannot exclude that patients without demonstrated reverse remodeling did not benefit clinically from $\mathrm{CRT}^{26}$. Finally, since we did not have any information on the exact conduction wavefronts between the pacing sites, we chose to only simulate latency at the LV pacing site as source of PLVD. Other factors that can lead to PLVD, such as anisotropic conduction related to more remote or diffuse fibrosis, may result in similar interventricular activation dynamics and thereby acute hemodynamic response. Furthermore, the computer simulations only describe the acute hemodynamic response to CRT. Similarly, the presence of myocardial scar or ischemia has not been simulated in this study.

\section{Conclusion}

In addition to the intrinsic electrical substrate, PLVD is strongly associated with less LV reverse remodeling, demonstrating that measuring the electrical substrate during pacing has additional value for prediction of CRT response in an already well-selected patient population. Computer simulations corroborate this finding by showing that, while intrinsic electrical dyssynchrony is a prerequisite, the level of pacing-induced dyssynchrony modulates acute CRT response. 


\section{References}

1. Brignole M, Auricchio A, Baron-Esquivias G, et al. 2013 ESC Guidelines on cardiac pacing and cardiac resynchronization therapy: Addenda The Task Force on cardiac pacing and resynchronization therapy of the European Heart Rhythm Association(EHRA). Eur Heart J. 2013;ESC Guidel:1-21. doi:10.1093/eurheartj/eht180

2. Prinzen FW, Vernooy K, Auricchio A. Cardiac resynchronization therapy: State-of-the-art of current applications, guidelines, ongoing trials, and areas of controversy. Circulation. 2013;128(22):2407-2418. doi:10.1161/CIRCULATIONAHA.112.000112

3. Auricchio A, Prinzen FW. Non-Responders to Cardiac Resynchronization Therapy. Circ J. 2011;75(3):521-527. doi:10.1253/circj.CJ-10-1268

4. Thibault B, Harel F, Ducharme A, et al. Cardiac resynchronization therapy in patients with heart failure and a QRS complex $<120$ milliseconds: The evaluation of resynchronization therapy for heart failure (LESSER-EARTH) trial. Circulation. 2013;127(8):873-881. doi:10.1161/CIRCULATIONAHA.112.001239

5. Gold MR, Birgersdotter-Green U, Singh JP, et al. The relationship between ventricular electrical delay and left ventricular remodelling with cardiac resynchronization therapy. Eur Heart J. 2011;32(20):2516-2524. doi:10.1093/eurheartj/ehr329

6. Stabile G, D'Onofrio A, Pepi P, et al. Interlead anatomic and electrical distance predict outcome in CRT patients. Hear Rhythm. 2015;12(11):2221 2229. doi:10.1016/j.hrthm.2015.05.020

7. Stephansen C, Sommer A, Kronborg MB, Jensen JM, Bouchelouche K, Nielsen JC. Electrically guided versus imaging-guided implant of the left ventricular lead in cardiac resynchronization therapy: a study protocol for a double-blinded randomized controlled clinical trial (ElectroCRT). Trials. 2018;19(1):600. doi:10.1186/s13063-018-2930-y

8. Sassone B, Gabrieli L, Saccà S, et al. Value of right ventricular-left ventricular interlead electrical delay to predict reverse remodelling in cardiac resynchronization therapy: The INTER-V pilot study. Europace. 2010;12(1):78-83. doi:10.1093/europace/eup347

9. Huntjens PR, Ploux S, Strik M, et al. Electrical Substrates Driving Response to Cardiac Resynchronization Therapy. Circ Arrhythmia Electrophysiol. 2018;11(4):e005647. doi:10.1161/CIRCEP.117.005647

10. Arts T, Delhaas T, Bovendeerd P, Verbeek X, Prinzen F. Adaptation to mechanical load determines shape and properties of heart and circulation: the CircAdapt model. Am J Physiol Heart Circ Physiol. 2005;288:19431954. doi:10.1152/ajpheart.00444.2004. 
11. Lumens J, Ploux S, Strik M, et al. Comparative electromechanical and hemodynamic effects of left ventricular and biventricular pacing in dyssynchronous heart failure: Electrical resynchronization versus left-right ventricular interaction. J Am Coll Cardiol. 2013;62(25):2395-2403. doi:10.1016/j.jacc.2013.08.715

12. Fornwalt BK, Sprague WW, BeDell P, et al. Agreement Is Poor Among Current Criteria Used to Define Response to Cardiac Resynchronization Therapy. Circulation. 2010;121(18):1985-1991. doi:10.1161/CIRCULATIONAHA.109.910778

13. Caputo ML, van Stipdonk A, Illner A, et al. The definition of left bundle branch block influences the response to cardiac resynchronization therapy. Int J Cardiol. 2018;269:165-169. doi:10.1016/j.ijcard.2018.07.060

14. Walmsley J, Arts T, Derval N, et al. Fast Simulation of Mechanical Heterogeneity in the Electrically Asynchronous Heart Using the MultiPatch Module. PLoS Comput Biol. 2015;11(7):e1004284.

doi:10.1371/journal.pcbi.1004284

15. Herweg B, Ali R, llercil A, et al. Site-specific differences in latency intervals during biventricular pacing: Impact on paced QRS morphology and echooptimized V-V interval. PACE - Pacing Clin Electrophysiol. 2010;33(11):1382-1391. doi:10.1111/j.1540-8159.2010.02882.x

16. Derval N, Duchateau J, Mahida S, Eschalier R. Distinctive Left Ventricular Activations Associated With ECG Pattern in Heart Failure Patients. 2017:1 10. doi:10.1161/CIRCEP.117.005073

17. Satoh H, Sano M, Suwa K, et al. Distribution of late gadolinium enhancement in various types of cardiomyopathies: Significance in differential diagnosis, clinical features and prognosis. World J Cardiol. 2014;6(7):585-601. doi:10.4330/wjc.v6.i7.585

18. Ploux S, Eschalier R, Whinnett Zl, et al. Electrical dyssynchrony induced by biventricular pacing: Implications for patient selection and therapy improvement. Hear Rhythm. 2015;12(4):782-791. doi:10.1016/j.hrthm.2014.12.031

19. Ploux S, Lumens J, Whinnett Z, et al. Noninvasive electrocardiographic mapping to improve patient selection for cardiac resynchronization therapy: beyond QRS duration and left bundle branch block morphology. J Am Coll Cardiol. 2013;61(24):2435-2443. doi:10.1016/j.jacc.2013.01.093

20. Auricchio A, Fantoni C, Regoli F, et al. Characterization of Left Ventricular Activation in Patients with Heart Failure and Left Bundle-Branch Block. Circulation. 2004;109(9):1133-1139. doi:10.1161/01.CIR.0000118502.91105.F6 
21. Garrigue $S$, Bordachar $P$, Reuter S, Jaïs $P$, Haïssaguerre $M$, Clementy J. Comparison of permanent left ventricular and biventricular pacing in patients with heart failure and chronic atrial fibrillation: a prospective hemodynamic study. Card Electrophysiol Rev. 2003;7(4):315-324. doi:10.1023/B:CEPR.0000023167.11038.8f

22. Yokoshiki H, Mitsuyama H, Watanabe M, Mitsuhashi T, Shimizu A. Cardiac resynchronization therapy in ischemic and non-ischemic cardiomyopathy. $J$ Arrhythmia. 2017;33(5):410-416. doi:10.1016/j.joa.2017.03.002

23. Gold MR, Singh JP, Ellenbogen KA, et al. Interventricular Electrical Delay Is Predictive of Response to Cardiac Resynchronization Therapy. JACC Clin Electrophysiol. 2016;2(4):438-447. doi:10.1016/j.jacep.2016.02.018

24. Zucchelli G, Soldati E, Di Cori A, et al. Role of intraoperative electrical parameters in predicting reverse remodelling after cardiac resynchronization therapy and correlation with interventricular mechanical dyssynchrony. Eur Eur pacing, arrhythmias, Card Electrophysiol J Work groups Card pacing, arrhythmias, Card Cell Electrophysiol Eur Soc Cardiol. 2010;12(10):1453-1459. doi:10.1093/europace/euq267

25. D'ONOFRIO A, BOTTO G, MANTICA M, et al. Incremental Value of Larger Interventricular Conduction Time in Improving Cardiac Resynchronization Therapy Outcome in Patients with Different QRS Duration. J Cardiovasc Electrophysiol. 2014;25(5):500-506. doi:10.1111/jce.12381

26. Wikstrom G, Blomstrom-Lundqvist C, Andren B, et al. The effects of aetiology on outcome in patients treated with cardiac resynchronization therapy in the CARE-HF trial. Eur Heart J. 2009;30(7):782-788.

doi:10.1093/eurheartj/ehn577 


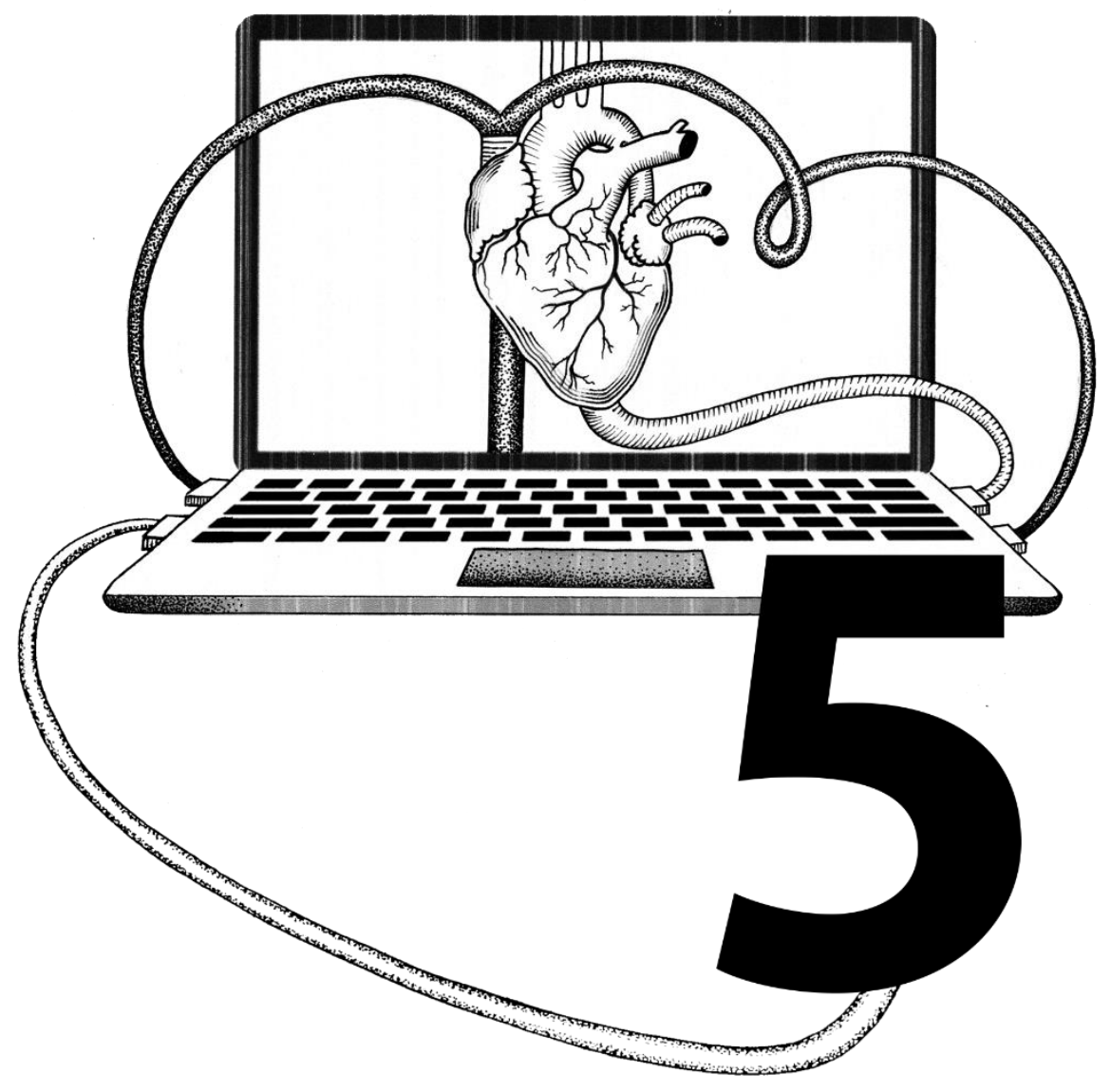




\section{Chapter 5}

\section{LBBB and High Afterload A Dangerous Liaison?}

Frits W. Prinzen, PhD, Erik Willemen, MSc, Joost Lumens, PhD

J Am Coll Cardiol Img. 2019 Jun, 12 (6) 978-980. doi: 10.1016/j.jcmg.2017.12.002 

Left bundle branch block (LBBB) is rare in the general population $(\sim 0.5 \%)$; however, LBBB is fairly common in patients with chronic heart failure $(\sim 25 \%)^{1}$. These numbers indicate an association between LBBB and cardiovascular disease, including hypertension and heart failure ${ }^{2}$. These associations have raised the question of what is cause and what is consequence. Half a century ago, it was commonly believed that newly acquired LBBB was a hallmark of advanced hypertensive or ischemic heart disease. On the other hand, Breithardt and Breithardt reviewed literature evidencing that LBBB could promote development of heart failure ${ }^{3}$. The latter observations were substantiated 2 decades later in animal experiments, in which induction of LBBB in otherwise normal dogs led to ventricular dilatation, asymmetrical hypertrophy, and a reduction in left ventricular ejection fraction (LVEF) 4 . More recently, studies in patients who developed LBBB after valve replacement procedures confirmed a causal relation between LBBB and mortality ${ }^{5}$. Obviously, the success of cardiac resynchronization therapy, which acutely improves pump function by correction of (most of) the electrical disturbances, also strongly supports the negative functional consequences of $\mathrm{LBBB}^{6}$.

In an issue of iJACC, Aalen et al. ${ }^{7}$ present a very elegant study in patients and dogs that demonstrates that hearts with LBBB are more sensitive to increased left ventricular (LV) afterload than normal hearts. In asymptomatic patients with LBBB, afterload was increased by handgrip exercise and arm-cuff inflation, whereas in dogs, afterload was increased in a more controlled way by aortic constriction. Although in normal subjects, increased afterload reduced LVEF only mildly (7\%), this reduction was more pronounced in LBBB patients (14\%). The dog experiments showed that in particular, septal stretch during the ejection phase deteriorated at high afterload. Both in humans and in dogs, increased afterload in the LBBB heart created a clockwise rotation 
of the septal pressure-length loop, which indicates negative ("wasted") work generated by the septum.

Therefore, this study adds important information to the abovementioned discussion as to whether LBBB is the chicken or the egg in relation to heart failure and hypertension: maybe it is both! After all, if LBBB develops in hypertensive patients (whether caused by hypertension or not), it creates a disproportionately negative effect on pump function.

The strain measurements reported by Aalen et al. ${ }^{7}$ provide insight into the mechanism by which LBBB and high afterload synergistically cause deterioration in LV pump function. We can speculate about the mechanism as to why the reduction in function is seen especially in the septum. After discovery of abnormal strain patterns in asynchronous hearts, it was noted that the explanation for worsening of pump function could be sought largely in the inherent property of myocardial cells called length-dependent activation (also known as the Frank-Starling mechanism) ${ }^{8}$. The earliest-activated region (the septum in LBBB) starts to contract and shorten early, thereby stretching the later-activated LV lateral wall. After electrical activation of the LV lateral wall, its contraction is strong because of its pre-stretch, and this augmented contraction forces the septal myocardium to be stretched, even though it is still actively generating force itself. There is evidence that the reduced systolic septal shortening (or even stretching) directly relates to the loss in pump function in dyssynchronous hearts?.

A next step is to try to explain the further worsening of septal systolic function at high afterload. This could relate to the muscle property that contraction lasts longer when afterload is high. As a consequence, systolic stress in the LV lateral wall might remain elevated and could thereby stretch the septum more than when afterload is normal, thus amplifying mechanical dyscoordination. 
Support for this idea comes from simulations we conducted in the CircAdapt computer program to simulate mechanics of the heart and circulation ${ }^{10}$. Figure 1 illustrates pressure-strain loops that are comparable to the pressure-segment length loops in the paper by Aalen et al. ${ }^{7}$. Increasing afterload hardly affects the loops in the synchronous heart, where simulating a mechanical activation delay in the LV free wall leads to a small pressure-strain loop that starts to turn clockwise when afterload is increased (simulated by partial aortic occlusion). Importantly, the CircAdapt model contains only a few elementary muscle properties, of which length-dependent activation is an important one. Therefore, it seems highly likely that

Figure 1 - Pressure-Strain Diagrams From CircAdapt Simulations of Synchronously and Dyssynchronously Activated Heart Before and After Increasing Afterload Through Partial Aortic Valve Occlusion

\section{Synchronous}
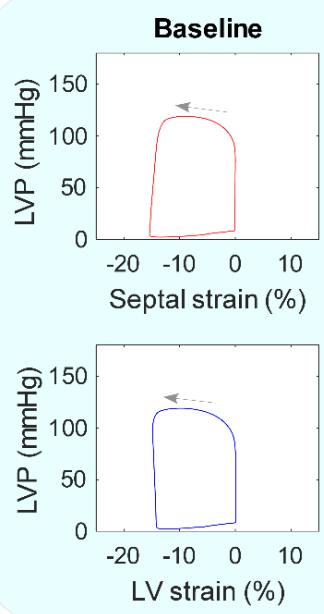
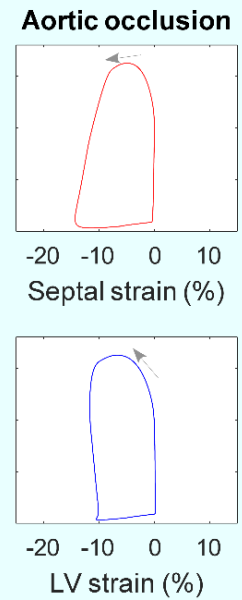

Dyssynchronous

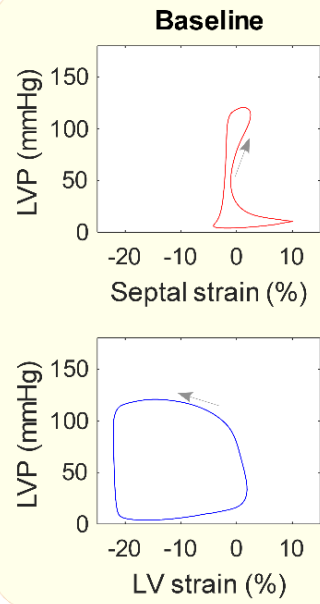

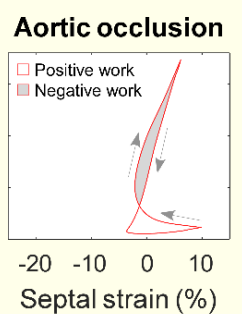

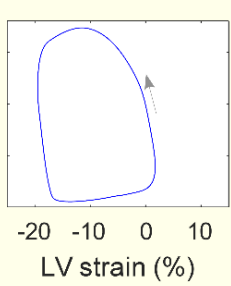

The MultiPatch version ${ }^{10}$ was used for these simulations, with segments for the right ventricular, septal, and left ventricular (LV) wall. During synchronous activation, all segments were activated simultaneously, whereas for dyssynchrony, activation of the septum and LV wall was delayed by 15 and 60 $m s$, respectively, with respect to the right ventricle. $\angle V P=$ left ventricular pressure. 
the afterload hypersensitivity of LBBB hearts can be (largely) explained by a com bination of basic muscle properties, interaction between ventricular walls, and the arterial afterload (Figure 1).

This likely explanation has at least 3 important clinical ramifications:

- Quantification of mechanical dyssynchrony and discoordination is sensitive to afterload. This has also been shown to some extent by Park et al. ${ }^{11}$, who used pneumatic leg compression and nitroglycerin administration to alter loading conditions. This implies that afterload is an important additional variable that should be known for proper interpretation of mechanical dyssynchrony data.

- Because LVEF also shows afterload hypersensitivity in patients with LBBB, blood pressure should be taken into account when interpreting LVEF measurements. This seems especially important when decisions are made based on a cutoff value, such as $35 \%$ for device implantation.

- Lowering the afterload in patients with LBBB might be even more favorable than in patients with narrow QRS complex and thus could be even more important for their proper treatment.

Of course, extrapolation of the results of this study to clinical care should be done with care. The study by Aalen et al. ${ }^{7}$ only investigated patients with LBBB without heart failure symptoms. Further studies are required to determine whether the afterload hypersensitivity also occurs in failing LBBB hearts. It would be equally interesting to investigate whether application of cardiac resynchronization therapy in such patients would reduce the afterload hypersensitivity. If this were to happen acutely, it would provide strong support for the mechanism of afterload hypersensitivity proposed above. 
Besides the small numbers of patients, a limitation of the study by Aalen et al. ${ }^{7}$ is its acute design. There is no information on the potential long-term effects. However, the "natural" adaptation of the myocardium under these conditions is usually counterproductive: long-term dyssynchrony and pump failure induce dilatation and asymmetrical hypertrophy, complicated by adverse molecular changes $^{4,12}$ and, through the cardiorenal axis, increased fluid maintenance. These all lead to further worsening of the condition of the patient. Therefore, the acute adverse effects of afterload could be amplified in the longer term. It is clearly of utmost importance to investigate this question in more detail.

In conclusion, the article by Aalen et al. ${ }^{7} \quad$ could be an important piece of the puzzle of understanding dyssynchronous heart failure, a piece that indicates that LBBB and LV pressure overload form a dangerous liaison, becoming hazardous at a much earlier stage than dangerous liaisons in fictional stories usually do. 


\section{References}

1. Baldasseroni S, Opasich C, Gorini M, et al. Left bundle-branch block is associated with increased 1-year sudden and total mortality rate in 5517 outpatients with congestive heart failure: A report from the Italian Network on Congestive Heart Failure. Am Heart J. 2002;143(3):398-405. doi:10.1067/mhj.2002.121264

2. SCHNEIDER JF. Newly Acquired Left Bundle-Branch Block: The Framingham Study. Ann Intern Med. 1979;90(3):303. doi:10.7326/00034819-90-3-303

3. Breithardt G, Breithardt OA. Left bundle branch block, an old-new entity. J Cardiovasc Trans/ Res. 2012;5(2):107-116. doi:10.1007/s12265-011-9344-5

4. Vernooy K, Verbeek XAAM, Peschar M, et al. Left bundle branch block induces ventricular remodelling and functional septal hypoperfusion. Eur Heart J. 2005;26(1):91-98. doi:10.1093/eurheartj/ehi008

5. Houthuizen P, Van Garsse LAFM, Poels TT, et al. Left bundle-branch block induced by transcatheter aortic valve implantation increases risk of death. Circulation. 2012;126(6):720-728.

doi:10.1161/CIRCULATIONAHA.112.101055

6. Lumens J, Ploux S, Strik M, et al. Comparative electromechanical and hemodynamic effects of left ventricular and biventricular pacing in dyssynchronous heart failure: Electrical resynchronization versus left-right ventricular interaction. J Am Coll Cardiol. 2013;62(25):2395-2403. doi:10.1016/j.jacc.2013.08.715

7. Aalen J, Storsten P, Remme EW, et al. Afterload Hypersensitivity in Patients With Left Bundle Branch Block. JACC Cardiovasc Imaging. 2018. doi:10.1016/j.jcmg.2017.11.025

8. Prinzen FW, Augustijn $\mathrm{CH}$, Arts T, Allessie MA, Reneman RS. Redistribution of myocardial fiber strain and blood flow by asynchronous activation. Am J Physiol - Hear Circ Physiol. 1990;259(2 28-2).

doi:10.1152/ajpheart.1990.259.2.h300

9. De Boeck BWL, Teske AJ, Meine M, et al. Septal rebound stretch reflects the functional substrate to cardiac resynchronization therapy and predicts volumetric and neurohormonal response. Eur J Heart Fail. 2009;11(9):863871. doi:10.1093/eurjhf/hfp107

10. Walmsley J, Arts T, Derval N, et al. Fast Simulation of Mechanical Heterogeneity in the Electrically Asynchronous Heart Using the MultiPatch Module. PLoS Comput Biol. 2015;11(7):e1004284.

doi:10.1371/journal.pcbi.1004284 
11. Park HE, Chang S-A, Kim H-K, et al. Impact of Loading Condition on the 2D Speckle Tracking-Derived Left Ventricular Dyssynchrony Index in Nonischemic Dilated Cardiomyopathy. Circ Cardiovasc Imaging. 2010;3(3):272-281. doi:10.1161/CIRCIMAGING.109.890848

12. Aiba T, Hesketh GG, Barth AS, et al. Electrophysiological Consequences of Dyssynchronous Heart Failure and Its R Therapy. Circulation. 2009;119(9):1220 doi:10.1161/CIRCULATIONAHA.108.7? 


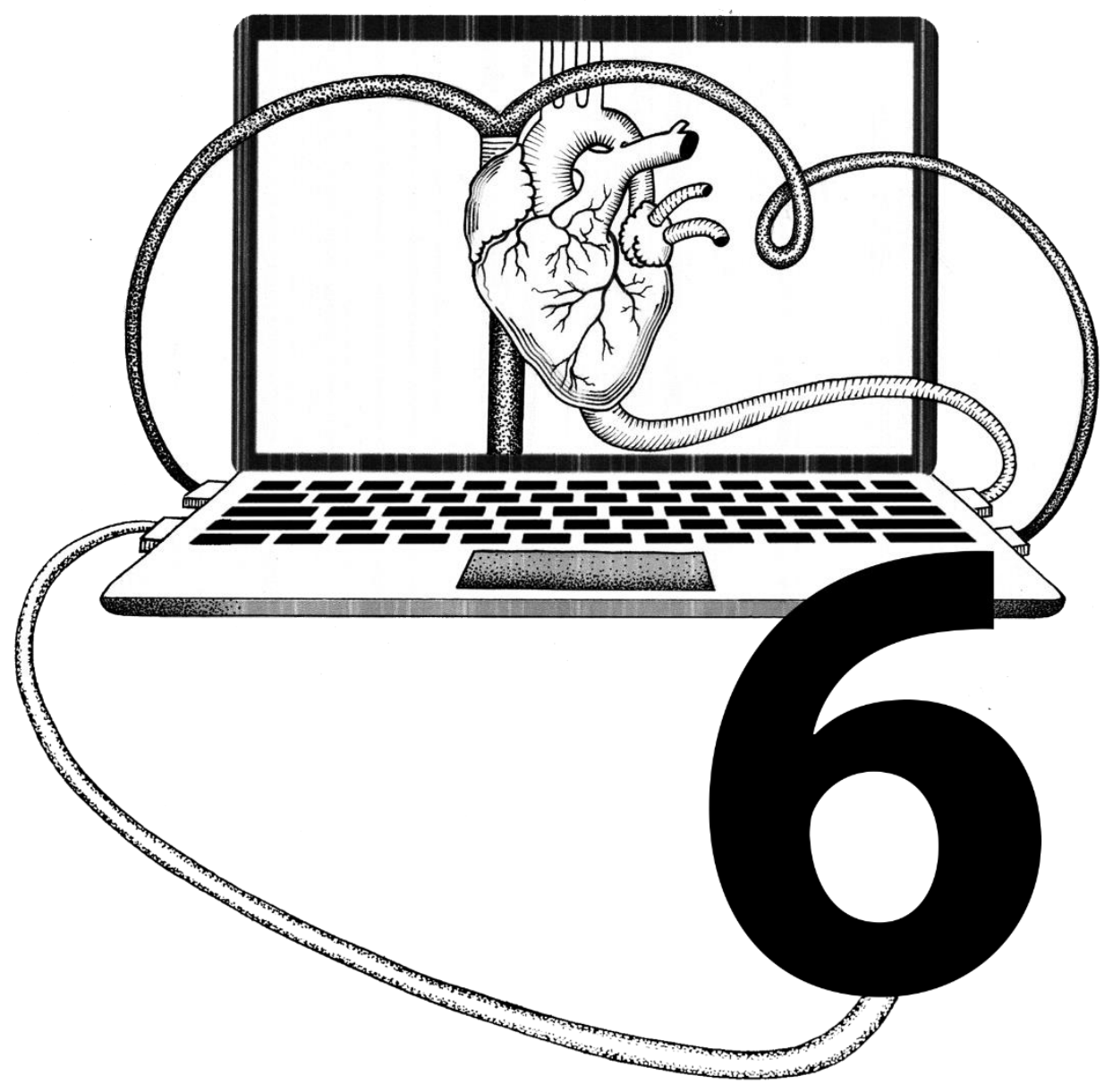




\section{Chapter 6}

\section{Does the Right Go Wrong}

During Cardiac Resynchronization Therapy?

Joost Lumens, PhD, Erik Willemen, MSc, Frits W. Prinzen, PhD

JACC Cardiovasc Imaging . 2020 Jul;13(7):1485-1488. doi: 10.1016/j.jcmg.2020.01.009 

About 1 in 3 patients with chronic heart failure (HF) has left bundle branch block (LBBB) ${ }^{1}$, a ventricular conduction disorder causing a distinct pattern of electromechanical polarization in the left ventricular (LV) wall, characterized by a decrease of work load in the early activated septum and an increase of work load in the late activated LV lateral wall ${ }^{2-}$ ${ }^{4}$. Many clinical studies have shown that LBBB is associated with increased morbidity and mortality ${ }^{5,6}$. The introduction of cardiac resynchronization therapy (CRT) has significantly improved the prognosis of patients with HF with reduced LV ejection fraction and hallmarks of LBBB on 12-lead electrocardiogram (i.e., widening and LBBB configuration of the QRS complex) $)^{7}$. The most popular working mechanism attributed to CRT is its instantaneous recoordinating effect on LV electromechanical function. Through biventricular pacing, CRT can (partially) repair the LV mechanical imbalance by inter- and intraventricular resynchronization of the electric activation ${ }^{8}$.

\section{A call for focus on right ventricular mechanical function}

It is important to realize that the vast majority of the studies investigating LBBB and its treatment with CRT has focused on LV mechanics and pump function. The right ventricle, however, is often dis- regarded in these studies, although it has been known for a long time that a change in the loading condition of either ventricle directly influences the pump function of the other ventricle. Also, several studies have identified right ventricular (RV) systolic dysfunction as an independent predictor of nonresponse to $\mathrm{CRT}^{9}$. For this reason, the publication of Storsten et al. ${ }^{10}$ in iJACC is important, as it provides mechanistic insight into the effects of both LBBB and CRT on RV mechanical function. The work is composed of an elegant combination of ani- mal experimental and human measurements. Echocardiographic longitudinal strain analysis in a group of patients with LBBB with nonischemic cardiomyopathy and 
normal RV function revealed an abnormal contraction pattern in the RV free wall, characterized by premature systolic shortening before RV ejection. In most patients, this early-systolic RV free wall shortening was significantly reduced or entirely abolished by CRT.

Storsten et al. ${ }^{10}$ observed the same premature RV systolic shortening in an animal model as soon as LBBB was induced by radiofrequency ablation of the left bundle branch. Because the intrinsic His-Purkinje conduction of the right ventricle was maintained in this acute model of LBBB, it was concluded that the RV contraction abnormality was a result of a change in direct LV-RV interaction. Interestingly, they also investigated what this change of ventricular interaction means for the distribution of work load over the ventricular walls.

\section{Myocardial unloading and reloading during $L B B B$ and CRT}

By combining LV and RV pressure measurements with myocardial deformation analysis using sonomicrometric crystals, the distribution of myocardial work across the ventricular walls was determined during LBBB and CRT. These experimental data corroborate previous experimental and clinical studies 3,11 by showing that LBBB increases myocardial work load in the late activated LV lateral wall and decreases work load in the early activated septum and that CRT (partially) restores the distri- bution of myocardial work load. Furthermore, the animal experimental data validate previous computer simulation work showing that $\mathrm{LBBB}$ reduces work load in the RV free wall and that CRT reverts this unloading effect (3). In other words, CRT instantly increases the work load of the RV myocardium in a patient with HF with LBBB. Therefore, as Storsten et al. ${ }^{10}$ suggest, their study raises the important question whether this pacing-induced change in RV work load can explain the association between base- line RV dysfunction and worse outcome after CRT. 


\section{Failure}

LVEF 33\%

RVEF $65 \%$
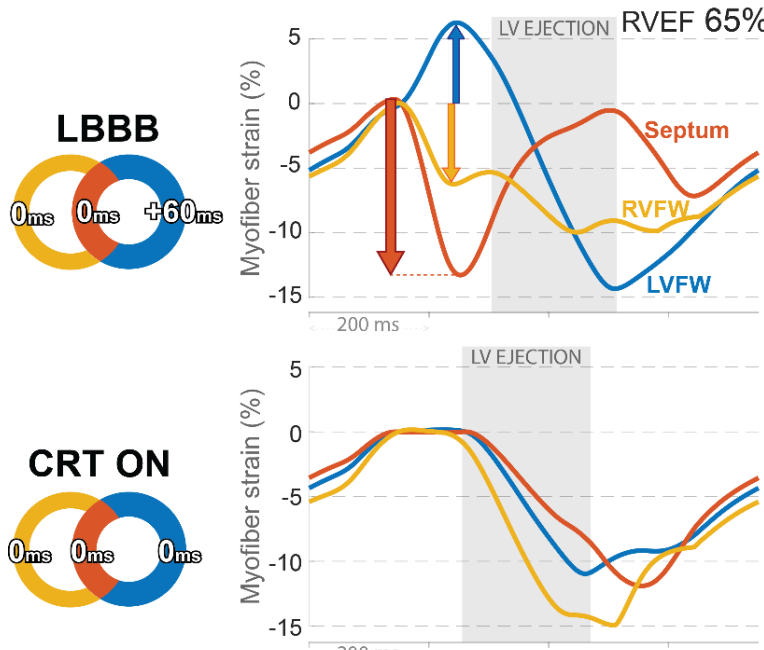

LV EJECTION

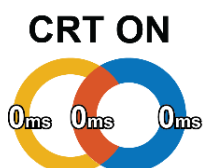

LV \& RV Failure

LVEF 33\%

RVEF 31\%
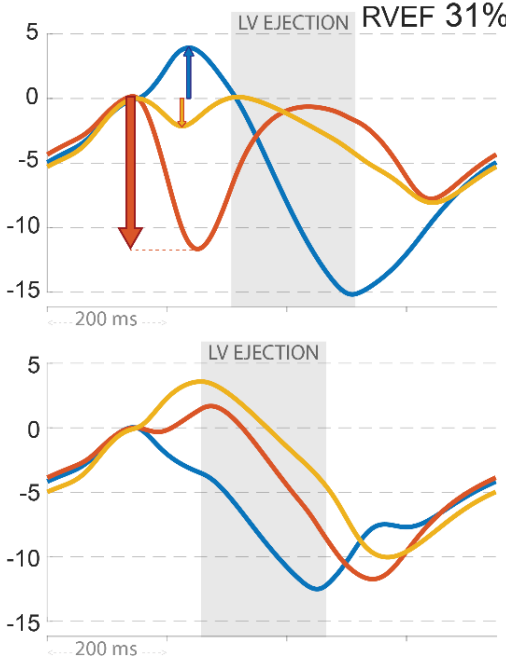

ACUTE CRT RESPONSE

$\triangle$ Cardiac Output
$+15 \%$ difference due to $\longrightarrow+3 \%$

Figure 1: Simulations of myofiber strain in the three ventricular walls during left bundle branch block ( $L B B B$, top row) and cardiac resynchronization therapy (CRT ON, middle row) in a heart with left ventricular (LV) failure (left column) and in a heart with both $L V$ and right ventricular (RV) failure (right column).

Simulations were performed with the CircAdapt model of human cardiovascular system dynamics (www.circadapt.org). LBBB was simulated by delaying mean LV free wall activation time $60 \mathrm{~ms}$ relative to mean septal and $R V$ free wall activation times. CRT was simulated by acutely synchronizing mean activation times of all three ventricular walls at an atrioventricular delay of $132 \mathrm{~ms}$. The bottom row presents CRT response for both $\angle B B B$ hearts. CRT response is defined as the acute pacing-induced change of cardiac output relative to the baseline cardiac output of 4,1 L/min in the LBBB simulations. Heart rate was kept constant at 80 bpm during all simulations. $L V E F=L V$ Ejection Fraction; LVFW $=$ LV Free Wall; $R V E F=R V$ Ejection Fraction; RVFW $=$ RV Free Wall. 


\section{Is RV work load the link between RV failure and nonresponse to CRT?}

For this editorial, we have performed computer simulations to test this hypothesis. We have used the well-established CircAdapt model of the human heart and circulation (CircAdapt, Maastricht University, Maastricht, the Netherlands), which has been extensively used for simulation of mechanics and hemodynamic status in the failing heart with $L B B B$ and during its treatment with $C R T^{3,12}$. Figure 1 shows the simulated strain patterns of the 3 ventricular walls during LBBB (Figure 1, top row) and during CRT (Figure 1, middle row) in a heart with LV failure and normal RV function (Figure 1, left column) and in a heart with both LV and RV failure (Figure 1, right column). The LBBB simulation with LV failure shows the same early systolic RV and septal shortening and LV pre-stretch as observed by Storsten et al. ${ }^{10}$ in dogs and HF patients with LBBB. In addition, the simulations predict that RV failure significantly reduces the amount of early systolic RV shortening and LV pre-stretch in LBBB. In the simulations of CRT (Figure 1, middlerow), the 3 ventricular walls are synchronously activated. As expected, CRT made the strain patterns of the 3 ventricular walls rather uniform in the heart with LV failure alone. Interestingly, CRT did not lead to such mechanical equilibration in the heart with additional RV failure. Instead, the LV free wall strain pattern showed early systolic shortening, and the RV freewall and septum were stretched before the onset of LV ejection. Given the synchronous electric activation of the 3 ventricular walls during CRT, this mechanical imbalance can be explained only by a marked interventricular difference in contractile strength during early systole, which is due to the pacing-induced redistribution of myocardial work load in the LBBB heart from the LV free wall to the RV free wall. The failing right ventricle cannot handle this acute increase in mechanical work load. The bottom panel of Figure 1 illustrates the effect of RV failure on hemodynamic response to CRT, in terms of acute change of 
cardiac output relative to baseline LBBB conduction. The simulations predict that RV contractile failure significantly reduces the potential for acute CRT response.

\section{RV deformation as a predictive biomarker}

In an earlier study, Van Everdingen et al. ${ }^{13}$ showed that conventional LV mechanical dyssynchrony as indexes (i.e., time to peak strain between the septum and LV lateral wall, interventricular mechanical delay, and septal systolic rebound stretch)do not reflect the negative impact of RV contractile dysfunction on CRT response. The data published by Storsten et al. ${ }^{10}$ and the simulations presented in this editorial suggest that the early systolic RV shortening can be of prognostic value, given its sensitivity to the electromechanical LBBB substrate that is amenable to CRT and to the contractile function of the RV free wall, which seems to be a strong determinant of CRT response.

\section{Going the right way with CRT}

Taking all these data together, we strongly support the notion by Storsten et al. ${ }^{10}$ that the diagnostic work-up of patients with HF who are candidates for CRT should include the assessment of baseline RV function. Their combination of patient and animal experimental data as well as the aforementioned computer simulations indicate that the RV free wall, which is mechanically spared in the LBBB heart, should be strong enough to bear the sudden increase in work load imposed by CRT. Future studies are needed to determine how much of the current problem of nonresponse to CRT is due to a lack of RV contractile reserve. 


\section{References}

1. Clark AL, Goode K, Cleland JGF. The prevalence and incidence of left bundle branch block in ambulant patients with chronic heart failure. Eur J Heart Fail. 2008;10(7):696-702. doi:10.1016/j.ejheart.2008.05.001

2. Gjesdal O, Remme EW, Opdahl A, et al. Mechanisms of abnormal systolic motion of the interventricular septum during left bundle-branch block. Circ Cardiovasc Imaging. 2011;4(3):264-273.

doi:10.1161/CIRCIMAGING.110.961417

3. Lumens J, Ploux S, Strik M, et al. Comparative electromechanical and hemodynamic effects of left ventricular and biventricular pacing in dyssynchronous heart failure: Electrical resynchronization versus left-right ventricular interaction. J Am Coll Cardiol. 2013;62(25):2395-2403. doi:10.1016/j.jacc.2013.08.715

4. Vernooy K, Verbeek XAAM, Peschar M, et al. Left bundle branch block induces ventricular remodelling and functional septal hypoperfusion. Eur Heart J. 2005;26(1):91-98. doi:10.1093/eurheartj/ehi008

5. Baldasseroni S, Opasich C, Gorini M, et al. Left bundle-branch block is associated with increased 1 -year sudden and total mortality rate in 5517 outpatients with congestive heart failure: A report from the Italian Network on Congestive Heart Failure. Am Heart J. 2002;143(3):398-405. doi:10.1067/mhj.2002.121264

6. Houthuizen P, Van Garsse LAFM, Poels TT, et al. Left bundle-branch block induced by transcatheter aortic valve implantation increases risk of death. Circulation. 2012;126(6):720-728. doi:10.1161/CIRCULATIONAHA.112.101055

7. Zareba W, Klein H, Cygankiewicz I, et al. Effectiveness of cardiac resynchronization therapy by QRS morphology in the multicenter automatic defibrillator implantation trial-cardiac resynchronization therapy (MADIT-CRT). Circulation. 2011;123(10):1061-1072. doi:10.1161/CIRCULATIONAHA.110.960898

8. Prinzen FW, Vernooy K, DeBoeck BWL, Delhaas T. Mechano-energetics of the asynchronous and resynchronized heart. Heart Fail Rev. 2011;16(3):215-224. doi:10.1007/s10741-010-9205-3

9. Damy T, Ghio S, Rigby AS, et al. Interplay between right ventricular function and cardiac resynchronization therapy: An analysis of the care-HF trial (Cardiac resynchronization-heart Failure). J Am Coll Cardiol. 2013;61(21):2153-2160. doi:10.1016/j.jacc.2013.02.049

10. Storsten P, Aalen JM, Boe E, et al. Mechanical Effects on Right Ventricular Function From Left Bundle Branch Block and Cardiac Resynchronization Therapy. JACC Cardiovasc Imaging. 2020;13(7).

doi:10.1016/j.jcmg.2019.11.016 
11. Russell K, Eriksen M, Aaberge L, et al. A novel clinical method for quantification of regional left ventricular pressurestrain loop area: A non-invasive index of myocardial work. Eur Heart J. 2012;33(6):724-733. doi:10.1093/eurheartj/ehs016

12. Walmsley J, Arts T, Derval N, et al. Fast Simulation of Mechanical Heterogeneity in the Electrically Asynchronous Heart Using the MultiPatch Module. PLoS Comput Biol. 2015;11(7):e1004284. doi:10.1371/journal.pcbi.1004284

13. van Everdingen WM, Walmsley J, Cramer MJ, et al. Echocardiographic Prediction of Cardiac Resynchronization Therapy Response Requires Analysis of Both Mechanical Dyssynchrony and Right Ventricular Function: A Combined Analysis of Patient Data and Computer Simulations. J Am Soc Echocardiogr. 2017;30(10):1012-1020.e2. doi:10.1016/j.echo.2017.06.004 


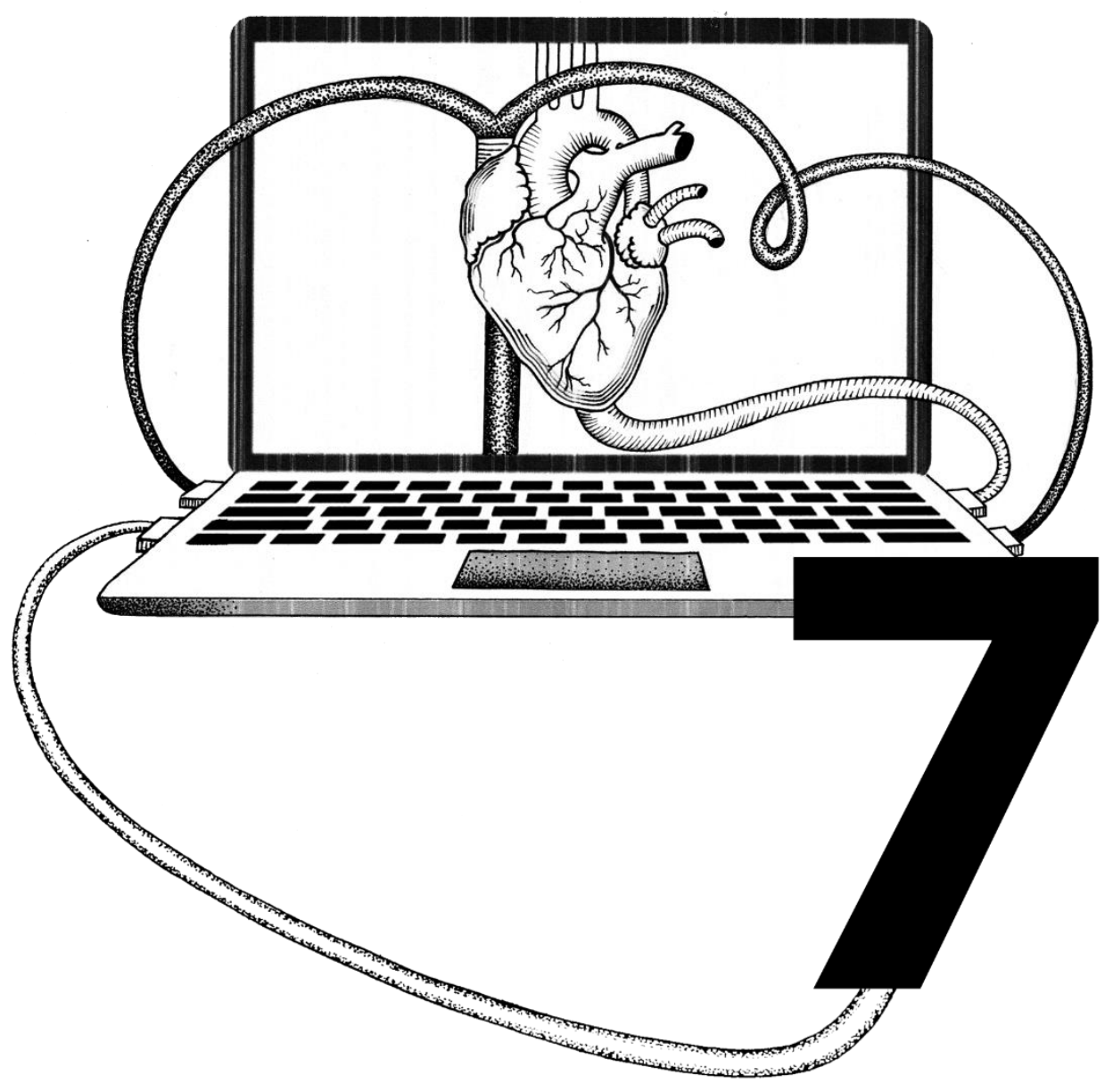


Chapter 7

General discussion 

The general aim of this thesis is to improve our insight into factors affecting response to pacing therapy. We did so by computationally assessing different aspects of cardiac pacing and evaluating the effect of various influences on cardiac deformation patterns.

In this thesis, cardiac pacing is studied in the context of both ventricular pacing against bradycardia (Chapter $\mathbf{2}$ ) and cardiac resynchronization therapy (CRT) (Chapter 3-6). During ventricular pacing the timing between atrial and ventricular contraction is affected by the atrioventricular delay ( $\mathrm{AVd}$ ) of the pacemaker. In Chapter 2 we showed that heart rate, inter-atrial delay and ventricular pacing site(s) affect the optimal AVd which delivers the best whole-heart pump function, in terms of cardiac output. The effective whole-heart AVd as percentage of the cardiac cycle length, incorporating activation times of both the left and right heart, enabled a more consistent definition of atrio-ventricular coupling.

In CRT, besides the AVd, the timing between ventricular contractions can also be affected by the ventriculo-ventricular delay ( $\mathrm{Vd}$ ) of the pacemaker. In Chapter 3 we combined animal-experimental and computer simulations over a wide range of $A V d$ and $\mathrm{VVd}$ settings and demonstrated that the left (LV) and right ventricle (RV) respond in an opposite manner to variations in LV or RV pacing delays, with RV contractility being optimal during RV pre-excitation and LV contractility being better at LV pre-excitation. Simulated cardiac output data, on the other hand, showed optimal values during (almost) simultaneous RV and LV pacing. In Chapter $\mathbf{4}$ we showed the importance of ventricular dyssynchrony during pacing in a combination of patient studies and computer simulations. We demonstrated that LV dyssynchrony during pacing is associated with lack of 6-month reverse remodeling in CRT patients.

The benefit of CRT not only depends on the settings of the pacemaker but also on the condition of the patient's heart. Echocardiographic 
measurements of ventricular deformation and myocardial work may be used to improve the selection of CRT candidates. In Chapter 5 we demonstrated that the typical pattern of deformation and myocardial work that exists during dyssynchronous ventricular activation is amplified when the same electrical dyssynchrony occurs at a higher LV afterload. Afterload is thus an important additional variable that should be known for proper interpretation of ventricular deformation data.

A further study showed that prediction of CRT response can further be improved by not only measuring LV deformation but also RV deformation. As shown in Chapter $\mathbf{6}$ a decrease in RV contractile reserve decreases the CRT response. Noninvasive measurement of RV deformation can help to identify patients with impaired RV function and thereby improve patient selection for CRT.

In this chapter, we place the results presented in the previous chapters into a broader perspective. The benefits of a whole-heart approach become apparent in the context of patient selection, therapy optimization, deformation imaging and computer modeling, leading to future perspectives that include clinical implications for the delivery of pacing therapy.

\section{Optimizing cardiac pacing}

LV function can improve acutely with changes in AVd and $\mathrm{V} d$ settings. However, no consensus exists on whether and how to perform the optimization of pacing delays and randomized studies did not systematically provide evidence for improved long term clinical outcome after optimization of pacing delays optimization in CRT patients ${ }^{1,2}$. No large clinical studies were found in which the long term clinical benefits of optimization of the AVd was studied in dual chamber pacing. Important in this regard is that all optimization studies targeted 
LV function measures, such as Doppler echo aortic VTI, mitral filling pattern and LV dP/dt $t_{\max }$. In this thesis, we assessed optimization of cardiac pacing in a whole-heart approach and demonstrated that a singular focus on the left heart may not be correct.

\section{Novel insights in AV optimization}

Several clinical studies on the effect of $A V d$ in dual chamber pacing and CRT found that the AVd at which ventricular filling is best also provides the best cardiac pump function ${ }^{3-7}$. Computer simulations in Chapter 2 demonstrated that the pacemaker setting that delivers optimal ventricular filling depends on inter-atrial and inter-ventricular activation as well as heart rate. The influence of inter-atrial delay and heart rate have been shown before ${ }^{3,8-10}$. Our finding that ventricular pacing site also affects the optimal $\mathrm{AVd}$ was new, as was the overarching concept of whole-heart effective $A V d$, incorporating these three factors. We think that our concept facilitates a more consistent definition of how the atria and ventricles interact optimally.

Future research may investigate whether relatively simple measurements like P-wave duration, inter-lead conduction times and/or interventricular mechanical dyssynchrony (speckle tracking or echoDoppler derived differences of aortic and pulmonary valve opening times) can be used to calculate whole-heart effective AVd. Ideally, the required measures should be implemented within a pacemaker, since that would enable continuous automatic optimization. Commercial optimization algorithms incorporating the electrograms derived from the pacing electrode exist. However, this approach did not show improvement over conventional echocardiographic optimization in large clinical trials ${ }^{11,12}$. The exact methodology of these algorithms is proprietary and therefore unknown. We think that a first step in creating a better algorithm would be to determine if pacing lead electrograms and/or echocardiographic measures can be used to reliably estimate effective whole-heart AVd. Such a study would require a 'gold-standard' 
for determining the effective whole-heart AVd such as an animal experimental or ex vivo setup including a large amount of electrodes on both the atria and ventricles. If a reliable estimate for effective wholeheart $A V d$ is found, a second study can investigate how this estimate should be normalized to heart rate in different patient populations. Based on these studies a third, very essential, study can investigate whether estimated effective whole-heart $A V d$, relative to heart rate, truly performs better than a fixed $A V d$, in terms of long-term clinical outcome after pacemaker implant.

\section{Novel insights in VV optimization}

The RV also plays a role in optimizing pacing settings in CRT. In Chapter 3,100 different combinations of $\mathrm{AVd}$ and $\mathrm{VVd}$ were tested in both animal experiments and computer simulations. As expected, ventricular electrical activation changed with variation in the amount of LV or RV pre-excitation. The resulting changes in $\mathrm{dP} / \mathrm{dt}_{\max }$ differed markedly between the LV and RV. Pacing the LV 10-50 ms before the RV led to the

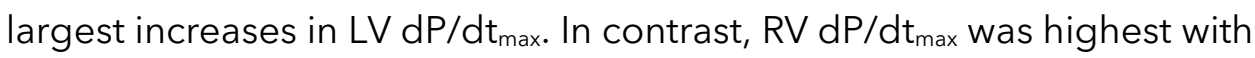
$\mathrm{RV}$ pre-excitation and decreased with LV pre-excitation. Simulations showed that the pacing-induced changes of whole-heart pump function, in terms of cardiac output, differed from the aforementioned changes in both LV and RV dP/dt $t_{\max }$. Therefore, improving LV contractility through LV pre-excitation does not necessarily lead to an improvement of global cardiac pump function. This can be understood from the serial coupling of the right and left side of the heart. For example, when a delay setting increases LV contractility but decreased RV contractility, less blood volume is pumped by the RV to the LV, lowering LV filling. Due to the decreased preload, the setting with the highest LV contractility might not necessarily result in an increased steady-state cardiac output. Steady-state whole-heart cardiac output was optimal during simultaneous pacing (i.e. $\mathrm{Vd}=0$ ), suggesting that optimal global cardiac pump function is a kind of compromise between left and right 
heart function. Measuring whole-heart steady-state cardiac output may be challenging in clinical practice, since the change of LV stroke volume in the first beat after a change of pacing have the highest signal-to-noise ratio ${ }^{13}$. Our results (Chapter 3 ) demonstrate that this higher signal-tonoise ratio might not necessarily result in a better determination of the optimum since the improvement in LV function, measured in these first beats, does not necessarily represent whole-heart improvement. Also, it is striking that several studies and authors describe to use LV preexcitation in CRT because this provides better LV function. Our data suggest that this is possibly not in the patients' interest. Another factor that has to be taken into account in optimization studies is that after a switch in pacing delays the baroreflex often counteracts the improvement or worsening of LV pump function. Future studies could investigate whether it is better to partially circumvent the baroreflex, through higher rate atrial pacing, or incorporate the rate response resulting from the baroreflex as measure of response.

Chapter 4 showed that during standard CRT, in a subgroup of patients LV dyssynchrony exists during biventricular pacing and that presence of such paced LV dyssynchrony (PLVD) is associated with less reverse remodeling after 6 months. PLVD was defined to be present if the time from $L V$ pace to $R V$ sense is longer than the time from RV pace to $L V$ sense. In patients with PLVD, pre-excitation of the LV might lead to an electrical ventricular activation that is similar to simultaneous pacing in patients without PLVD. This approach needs to be studied in order to test this hypothesis, especially since PLVD might also be related to presence of less viable tissue due to ischemia. However, this example demonstrates why a patient-specific approach towards optimization is necessary. Measuring conduction times at implant, as in Chapter 4, could help in finding patients with PLVD in which pacing optimization should be prioritized. The amount of PLVD can potentially be used to determine the amount of LV pre-excitation required. 


\section{Need for a combined whole-heart systems approach when optimizing pacing delays}

Our findings in Chapters $\mathbf{2}$ and $\mathbf{3}$ may provide an explanation why meta-analysis of $\mathrm{AV}$ and $\mathrm{V}$-delay optimization studies did not show any long-term benefits for optimization of pacing delays in CRT patients ${ }^{2}$. In all trials, AV and/or VV optimization were performed in series instead of in parallel. Often AVd optimization is performed first, followed by $\mathrm{Vd}$ optimization. Results from Chapter 2 showed that changes in interventricular delay, which also changes with $\mathrm{VVd}$, can change the effective whole-heart $A V d$ and thereby the optimal $A V d$. If the $V V d$ is changed while maintaining the same optimized paced $A V d$, ventricular filling will thus become suboptimal. This change in ventricular filling, resulting from the change in effective $A V d$, can also affect the measurement that is being used to optimize the $\mathrm{Vd}$. Therefore, it is important to maintain the same effective whole-heart AVd when optimizing the $\mathrm{Vd}$ so that the chosen measurement truly reflects the change in ventricular activation.

The measurements used for the optimization of pacing delay settings differ between studies. As demonstrated in Chapter 3, LV and RV $\mathrm{dP} / \mathrm{dt}_{\max }$ seem most susceptible to changes of contractility in their respective ventricle. Both in the animals and in CircAdapt $L V d P / d t_{\max }$ mostly depended on $\mathrm{VVd}$ but, improving $\mathrm{LV} \mathrm{dP} / \mathrm{dt}_{\max }$ could result in reduced $R V$ contractile function. Importantly, the highest $L V d P / d t_{\max }$ does not correlate with the largest whole-heart cardiac output. This model observations seems to be corroborated by findings in patients

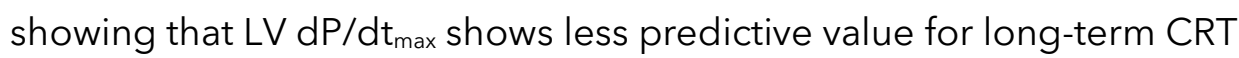
response compared to stroke work ${ }^{14}$. When designing optimization protocols it is important to realize that the hemodynamic response during the first beats after a change of pacing delay can differ substantially from the change in steady-state cardiac output that is eventually reached due to the balancing of ventricular preload during the next few beats. We did not study which hemodynamic outcome 
measure is more important for long-term outcome in patients, but our data demonstrate that the use of $\mathrm{LV} \mathrm{dP} / \mathrm{dt}_{\max }$ as optimization target should be done with care and that studies using it should be interpreted carefully. Combining measures of LV and RV output and/or taking measures in the steady-state, after the first 2-10 beats, will give a better representation of whole-heart function.

\section{A broader perspective based on insights from pacing optimization}

Our insights in optimization of pacing delays also result in proposals for clinical application of cardiac pacing outside of optimization of pacemaker settings. Currently, clinical criteria for CRT focus on patients already exhibiting adverse ventricular remodeling in combination with LV electrical dyssynchrony. At the same time, RV pacing is still standard therapy in patients with preserved ejection fraction receiving ventricular pacing for symptomatic bradycardia ${ }^{15}$. The results of Chapter 3 demonstrate that simultaneous biventricular pacing has the potential to provide higher cardiac output than RV- or LV-only pacing. Therefore, we agree with the suggestion presented in a review by Fang et al $^{16}$ and believe that RV pacing should be replaced as the clinical standard in patients who require frequent ventricular pacing, such as patients with complete AV block. Creating a physiological ventricular activation through pacing the His-Purkinje network provides the most physiological activation and would likely be best, but challenges still exist and the required scientific evidence in terms of randomized controlled trials is missing ${ }^{17}$. Benefits of His-bundle or biventricular pacing should always outweigh the risk of complications and lead dislodgement, thus a patient-specific risk-assessment is always warranted.

At the moment, cardiac pacing is recommended for either treatment of symptomatic bradycardia or ventricular dyssynchrony. The optimization chapters of this thesis suggest that ventricular filling, through atrioventricular coupling, plays a major role in the improvement of cardiac 
pump function with cardiac pacing. This raises the question whether cardiac pacing could be an effective therapy for patients suffering from chronic ventricular filling problems as a result of slow AV conduction, characterized by a long PR interval on the 12-lead ECG. The obvious downside of this is that ventricular pacing introduces some degree of ventricular dyssynchrony. In patients with a prolonged PR interval, the pacing-induced improvement in AV coupling might outweigh the negative effect on pump function of pacing-induced ventricular dyssynchrony, in case of biventricular pacing ${ }^{18}$. This is a hypothesis worth investigating, especially since modes of more physiological Hisbundle or left bundle-branch pacing are becoming clinically available ${ }^{19}$.

\section{Factors influencing ventricular deformation and its potential for CRT response prediction}

In dyssynchronously activated ventricles, early activated regions start to shorten before aortic valve opening, thereby stretching later activated regions. Once these late activated regions are activated, they contract more forcefully due to the local Frank-Starling effect. The stronger prestretched contraction causes mid-systolic stretching of the earlier activated regions. Part of the work performed by the ventricle in dyssynchronous hearts is thus used for stretching ventricular segments activated at another time, instead of building up ventricular pressure. During LBBB this leads to low, sometimes negative, myocardial work in the early activated septum and elevated in the late activated LV lateral wall ${ }^{20-22}$. Earlier studies demonstrated that this distribution of regional myocardial work is potentially related to adverse remodeling since it is related to both regional metabolism ${ }^{23}$ and changes in ventricular wall mass $^{22}$.

Ventricular deformation (stretching and shortening) can be measured with echocardiography and new developments also enable the noninvasive estimation of myocardial work by combining deformation 
measurements with estimated LV pressure ${ }^{24}$. Recent studies have suggested that this noninvasive assessment of LV regional work can help to predict CRT-response ${ }^{21,25}$. One of the reasons why quantification of regional ventricular strain and work can help in assessing the potential for CRT response is that deformation does not only depend on the electrical substrate but also on non-electrical properties of the myocardium, such as contractility and stiffness, which can modulate the potential for response to CRT. Therefore, patients with scar or other contractile problems present with different distributions of deformation and work compared to patients with the same amount of ventricular dyssynchrony but more viable tissue.

As shown by Aalen et al, quantification of mechanical dyssynchrony is also sensitive to LV afterload ${ }^{26}$. Computer simulations performed in Chapter $\mathbf{5}$ suggest that the afterload hypersensitivity of LBBB hearts can largely be explained by a combination of basic muscle properties and interaction between ventricular walls. Afterload dependence of myocardial deformation and work might complicate its use as a selection criterion for CRT, since the same amount of work is not indicative for the same amount of dyssynchrony in a population where hypertension is present in more than $60 \%$ of CRT and pacemaker patients ${ }^{27}$. In clinical practice, afterload is thus an important additional variable that should be known for proper interpretation of mechanical dyssynchrony data.

Other imaging-based measures used in the context of CRT are also sensitive to variations in afterload. For example, LVEF shows afterload hypersensitivity in patients with LBBB and therefore blood pressure should be taken into account when interpreting LVEF measurements ${ }^{26}$. This seems especially important when decisions for device implantation are made based on a cutoff value of $35 \%$. Two patients with the same electrical substrate, thereby having the same potential electrical benefit of CRT, according to today's guidelines could receive a different advice 
based on a different blood pressure at the moment the LVEF was measured. Additionally, lowering the afterload in patients with LBBB might be even more favorable than in patients with narrow QRS complex and thus could be even more important for their proper treatment.

\section{RV deformation in LV dyssynchrony}

Several studies have identified RV systolic dysfunction as an independent predictor for nonresponse to $\mathrm{CRT}^{28,29}$. In clinical practice, however, RV function is not taken into account when selecting patients for $\mathrm{CRT}^{15}$. Furthermore, studies that investigated the use of deformation imaging for improving the selection of CRT candidates have focused on the LV myocardial deformation only. We used computer simulations to study the effect of RV failure on ventricular deformation patterns during LBBB and CRT, thereby extending the work of Storsten et al who elegantly studied the effects of LBBB and CRT on RV mechanical function in animal and human experiments ${ }^{30}$.

Echocardiographic longitudinal strain analysis in a group of patients with LBBB with nonischemic cardiomyopathy and normal RV function revealed an abnormal contraction pattern in the RV free wall, characterized by premature systolic shortening before RV ejection ${ }^{30}$. Computer simulations confirmed the same RV deformation pattern during LV dyssynchrony (Chapter 6). Additionally, simulations predict that RV failure significantly reduces the amount of early systolic RV shortening and LV pre-stretch in LBBB. These same simulations showed that RV contractile failure significantly reduces the potential for acute CRT response. This lack of response was due to the inability of the RV to provide the additional workload that is shifted to the RV upon resynchronization. Early systolic RV shortening can be of prognostic value, given its sensitivity to the electromechanical LBBB substrate that is amenable to CRT and to the contractile function of the RV free wall, which is also a strong determinant of CRT response. Given this 
mechanistic relation between RV failure and non-response to CRT, the diagnostic work-up of patients with HF who are candidates for CRT should include the assessment of baseline RV function through deformation imaging. If deformation imaging is not possible, exercise testing might provide an alternative since exercise capacity is linked to RV function ${ }^{31}$. Furthermore, a low exercise capacity before CRT implant is related to non-response ${ }^{32}$, potentially because of RV dysfunction.

\section{A whole-heart assessment of deformation imaging in CRT}

The finding that viability of the $\mathrm{RV}$ tissue plays a role in the response to CRT (Chapter 6) illustrates that a whole-heart assessment is unavoidable in order to identify all possible factors that can limit the benefits of CRT. It also shows that RV function can directly affect LV deformation. Besides a whole-heart assessment being a prerequisite for a complete interpretation of ventricular deformation in a dyssynchronous HF context, the circulations which connect both sides of the heart also affect cardiac deformation and should thus be taken into account.

Deformation is thus not an easy one-size-fits-all solution but can certainly provide clinical value.

In patients with a mix of moderate dyssynchrony (QRS duration 120$150 \mathrm{~ms}$ ) and ischemic heart failure it can be hard to distinguish to what extent the dyssynchrony or mechanical tissue properties determine a deformation pattern. Strain-based indices like systolic stretch index ${ }^{33}$ do allow better characterization of underlying myocardial disease substrates than time-to-peak strain measurements. However, there remains room for improvement since generic cutoff values are unlikely to work for all patients under all conditions. We hypothesize that the afterload dependence of ventricular deformation patterns in dyssynchronous hearts (Chapter $\mathbf{5}$ ) can also be used to gain additional information about the extent of ventricular dyssynchrony within a patient. For this hypothesis we assume that changes related to ischemia respond differently to a change in afterload, an assumption that still has 
to be investigated. If this assumption is proven, a afterload challenge, for example through pneumatic compression of the lower extremities ${ }^{34}$, could prove to be a more robust method to identify deformation changes that result from an electrical dyssynchrony substrate.

\section{Computer simulations in cardiac pacing}

\section{Tool for hypothesis generation with potential for guiding future clinical decision making}

As demonstrated in this thesis, response to cardiac pacing is a multifactorial problem and therefore a challenging puzzle to solve. Mechanistic computer models provide an integrative platform which, in a simplified way, describes the complex non-linear dynamics of the cardiovascular system or subsystems, using physical and physiological principles. Since all aspects are quantitatively described within a model, specific system properties can be studied in isolation, i.e. without changes that would occur in the more uncontrolled in vivo setting. Therefore, simulations can be used to complement both animalexperimental and clinical trials, obtaining information not measured or even unmeasurable. Subsequently, simulations can help to improve the design of experimental and clinical studies, thereby reducing experimental cost and risk for animals and patients. In our view, collaboration between computer scientists, experimental and clinical researchers are a key component of this process since data from in vivo and in vitro experiments will be necessary to parameterize and validate the state of a model in a physiological manner. At the same time, this thesis shows that simulations can also extend findings from the experiments that were used for model validation (Chapter $\mathbf{3}$ ). 


\section{Advantage and Limitations of the CircAdapt model}

The advantage of CircAdapt in the context of cardiac pacing is that it incorporates all the chambers in the heart and the circulations connecting the right and left heart. Both the direct mechanical ventricular interaction as well as the serial coupling though the circulation are essential for the match between simulations and measurements as demonstrated in this thesis. The phenomenological representation of the Frank-Starling mechanism within CircAdapt's sarcomere module and assuming a simple geometry enable fast simulation without the need for thousands of elements. However, when more regional or anatomic differentiation and interaction are required, the CircAdapt model will not suffice and a more complex and computationally demanding finite-element approach will be necessary.

In this thesis, we used different definitions of response to pacing therapy, with the change in steady-state cardiac output being used the most. We also showed that different definitions of response to pacing therapy do not provide the same result. In animal-experimental and clinical studies it is not always possible to measure everything.

Sometimes the measurements that can be done can be extended through simulations, such as in Chapter 3. All computer simulations presented in this thesis were limited to the acute hemodynamic response to pacing therapy, for which it remains unclear whether it relates to chronic response measures, such as reverse remodeling, or to more clinical outcome measures, such as HF hospitalization or death. While we found both acute and long-term benefits of avoiding paced LV dyssynchrony in Chapter 4, this can hardly be considered evidence for a causal relation. Computer models could potentially provide more insight into the relation between acute and long-term response. Within our lab at Maastricht University, a cell-level model of electro-mechanical interaction ${ }^{35}$ is currently being coupled to the more macroscopic CircAdapt model of organ- and system-scale cardiovascular physiology. 
Upon completion, results from in vitro studies into ion channel pathophysiology can potentially be related to whole-heart function through simulations. Going from cellular function to whole-heart function is especially interesting for the modeling of growth and remodeling at the tissue level, which is essential when going from acute to long-term response.

\section{Improvements and alternatives for cardiac pacing simulations}

Many components of cardiac and circulatory mechanics and hemodynamics are present within the CircAdapt model. However, the model does not incorporate cellular interaction, electrical conduction, 3D anatomy and other components that may be relevant for (among others) cardiac pacing. In part these limitations can be circumvented by integration of CircAdapt with additional modules, depending on the research question asked. For example, the recently developed MechChem module describes sarcomere mechanics from a physiological instead of phenomenological perspective ${ }^{36}$. The more physiological perspective can help when a research question requires electro-mechanical coupling on a more microscopic level. Some questions, however, require very different types of models. For example, electrophysiological questions regarding transmural electrical conduction and/or conduction within the Purkinje network, could be addressed by models describing electrical propagation in more detail ${ }^{37-}$

${ }^{39}$. Chapter 3 demonstrated that the output of such electrical models can subsequently be used for simulations of hemodynamics and mechanics within CircAdapt. Since CircAdapt is based on the one-fiber model of sarcomere mechanics ${ }^{40}$, mechanical finite element can provide a solution when incorporation of myofiber orientation or transmural structural differences is required for a research question ${ }^{37-39,41}$.

Homeostatic regulation, as implemented in the CircAdapt model, changes the total circulating blood volume (and thereby preload) as well as systemic vascular resistance in order to reach pre-defined target 
values of cardiac output and mean arterial pressure, given a fixed heart rate. In this process, no limitations are placed on the total circulating blood volume or resistance. For computational studies on long-term response and more diverse pathologies, homeostatic regulation should be implemented in a more physiological way. For chronic response a more gradual change in systemic resistance and circulating volume could for example be studied, where the targeted cardiac output is not always reached if this is not (patho)physiologically possible. Allowing a change in heart rate should also be part of this improved homeostatic control. A physiologically correct, chronologically realistic homeostatic regulation would allow a more realistic input for sensing parameters at the tissue level which is needed to model realistic tissue growth and remodeling. Furthermore, a better implementation of homeostatic regulation would also allow more realistic simulation of exercise which is essential for a patient's quality of life and has additional potential for clinical application in exercise-stress testing.

While the homeostatic regulation relates to a relatively short term process, cardiac remodeling is a slower process that adjusts the mass of the myocardium to mechanical load. In dyssynchronous hearts the remodeling process is locally different ${ }^{22}$, most likely due to the extensive local differences in workload (see above). Kerckhoffs et al demonstrated that simulating ventricular remodeling in in the context of ventricular dyssynchrony is possible using a finite-element approach ${ }^{43}$. While adaptation can be implemented in CircAdapt through the ventricular adaptation module developed by Arts et a ${ }^{42}$, additional research is required to validate and/or adapt this module for dyssynchronous hearts. 


\section{Creating a 'digital twin': patient-specific simulation-assisted pacing therapy advice}

The 'digital twin' is a medical engineering concept that has gained interest in the field of clinical cardiology. A digital twin is an in silico representation of a physical system, i.e. an engine or a patient, used to optimize design or control processes, with a real-time connection between the physical system and the model ${ }^{44}$. If each patient would have his or her own perfect digital twin, the optimal treatment could always be administered since therapeutic response for all options could be simulated. The fact that this thesis could still be written means that there a still a long road ahead before the first perfect digital twin can be modeled. The research presented in this thesis did provide several important stones to pave the road towards a realistic personalized cardiovascular model: 1) realistic simulations of cardiac pacing requires a model with all four chambers of the heart describing their mechanical and hemodynamic interactions (Chapter 2, $\mathbf{3}$ and 6) and 2) both sides of the heart need to be connected through a circulation (Chapter 2, $\mathbf{3}$ and 5), forming a closed-loop system that can run for several beats until a hemodynamic steady-state is reached (Chapter 3). CircAdapt, as is, can already function as a platform for personalized cardiovascular modeling. Steps in model personalization, based on patient measurements, are currently under research within multiple projects at Maastricht University. However, a lot of work is still required before a CircAdapt based digital twin can truly help in clinical decision making. 


\section{Impact}

In the Netherlands a total of 12.176 pacemakers were implanted in 2018 45. The European average is a bit higher with 938 implants per million inhabitants in $2011^{46}$. A better understanding of cardiac pacing and pacing therapy delivery can therefore impact a large amount of people and society as a whole. By combining cardiovascular computer modeling with animal experimental and patient data, we aimed to improve our understanding of the working mechanism(s) of cardiac pacing and thereby facilitate optimal pacing delivery and patient selection. Here, we summarize the clinical impact of the findings reported in this thesis and address the impact our findings have on future scientific and computational modeling work.

\section{Clinical impact}

The results presented in this thesis provide opportunities for the improvement of pacing therapy delivery through optimization and selection through measurement of deformation patterns. Our finding that both optimization of cardiac pacing and selection of candidates for CRT require a whole-heart approach may have major clinical impact. While the right heart is often not taken into account in current clinical practice, Chapters 2, 3, and $\mathbf{6}$ demonstrate that the right heart does have a significant role in cardiac performance during pacing therapy. A singular focus on improving LV function through optimization of pacemaker settings might not provide the largest improvement in global cardiac pump function (Chapter 3 ). Taking into account both the right- and left atrial and ventricular activations can provide a universal $\mathrm{AVd}$ optimization target, applicable in all forms of cardiac pacing. Additional research, as suggested previously in this chapter and in Chapter 2, can provide insights in how to obtain this whole-heart activation data in clinical practice. In Chapter $\mathbf{4}$ we found that measurements of conduction times between the left and right 
ventricular pacing electrodes can predict LV reverse remodeling after 6 months. Performing these measurements at the time of implant is quick and easy, enabling rapid clinical implementation. Future research is required to check if $\mathrm{Vd}$ optimization (Chapter 3 ) can help to improve outcome in patients where non-response is predicted through measurement of PLVD.

As demonstrated in this thesis, echocardiographic measurement of myocardial deformation can be a powerful tool for selection of patients for CRT. Our simulations strongly suggest that LV afterload should be taken into account when interpreting deformation data in the context of ventricular dyssynchrony (Chapter 5). At the same time, acute manipulation of afterload could potentially extend the applicability of deformation imaging for the identification of ventricular dyssynchrony. In addition, including data on RV deformation in the diagnostic assessment of CRT candidates is advised based on the results from Chapter 6. RV deformation is similar to septal deformation in patients with LV dyssynchrony without RV failure, since both the RV and septum are early activated in these patients. Our results suggest that RV failure changes this pattern and that RV failure itself makes patients less likely to respond to CRT. Taking into account LV afterload and also assessing $\mathrm{RV}$ deformation can help in improving the selection for CRT patients which will have significant clinical impact.

\section{Computational assessment can make research more efficient, reducing animal burden}

The work presented in this thesis demonstrates how animal experimental and/or clinical research studies can be enhanced through simulations. For example, the simulated cardiac output data in Chapter $\mathbf{3}$ add an important level of detail that was not measurable in a sufficiently accurate manner in the animal experiments. Knowledge about the overall cardiac pump function, in terms of cardiac output, enabled conclusions which were not possible based on the 
experimental pressure data alone. Additionally, in Chapter 2 we demonstrated that computer simulations alone can be used in hypothesis generation which hopefully leads to a new and improved focus in future clinical/experimental research studies. Multidisciplinary collaboration between scientists from different but complementary fields, such as biomedical engineering, physiology and clinical cardiology, is key to harvest most potential from computational modeling and simulation. This includes indirect collaboration by building upon previous work, which is an essential part of checking validity of propositions and thereby the creation of scientific evidence. Striving for a more open scientific community, such as sharing the source code during publishing as in Chapter 3, can therefore speed up the scientific process. Data from animal experimental and clinical research is needed in order to develop and validate computer models. However, these same computer models requiring experimental data can help in reducing the amount of animal and clinical studies required by improving hypotheses and related research protocols. Furthermore, once a computer model is sufficiently validated, and thereby credible, parts of the research process can be performed through computer simulations in 'in silico trials' ${ }^{\prime 4}$, potentially significantly reducing costs and time. 


\section{Conclusion}

Computer modeling can be a valuable tool to unravel whole-heart and circulatory interactions that play a role in the working mechanism of cardiac pacing therapy. The combination of computer simulations with animal experimental and clinical data showed that measurements providing insight in whole-heart activation are important for determining the patient's potential for positive response to cardiac pacing. Measurement of left ventricular deformation alone provides incomplete insight into ventricular electrical dyssynchrony, since right ventricular function and LV afterload also modulate the mechanical discoordination in dyssynchronous hearts. Therefore, an integrative whole-heart assessment is the key to achieve optimal response in patients receiving cardiac pacing therapy. 


\section{References}

1. Sohaib SMA, Whinnett ZI, Ellenbogen KA, et al. Cardiac resynchronisation therapy optimisation strategies: Systematic classification, detailed analysis, minimum standards and a roadmap for development and testing. Int J Cardiol. 2013;170(2):118-131. doi:10.1016/j.ijcard.2013.10.069

2. Auger D, Hoke U, Bax JJ, Boersma E, Delgado V. Effect of atrioventricular and ventriculoventricular delay optimization on clinical and echocardiographic outcomes of patients treated with cardiac resynchronization therapy: A metaanalysis. Am Heart J. 2013;166(1):20-29. doi:10.1016/j.ahj.2013.03.021

3. Mehta D, Gilmour S, Ward DE, Camm AJ. Optimal atrioventricular delay at rest and during exercise in patients with dual chamber pacemakers: a non-invasive assessment by continuous wave Doppler. Heart. 1989;61(2):161-166. doi:10.1136/hrt.61.2.161

4. Manisty $\mathrm{CH}, \mathrm{Al}-$ Hussaini A, Unsworth $\mathrm{B}$, et al. The acute effects of changes to AV delay on BP and stroke volume: potential implications for design of pacemaker optimization protocols. Circ Arrhythm Electrophysiol. 2012;5(1):122-130. doi:10.1161/CIRCEP.111.964205

5. Wish M, Fletcher RD, Gottdiener JS, Cohen Al. Importance of left atrial timing in the programming of dual-chamber pacemakers. Am J Cardiol. 1987;60(7):566571. doi:10.1016/0002-9149(87)90306-7

6. Whinnett ZI, Davies JER, Willson K, et al. Determination of optimal atrioventricular delay for cardiac resynchronization therapy using acute noninvasive blood pressure. Eur Eur pacing, arrhythmias, Card Electrophysiol J Work groups Card pacing, arrhythmias, Card Cell Electrophysiol Eur Soc Cardiol. 2006;8(5):358-366. doi:10.1093/europace/eul017

7. Auricchio A, Stellbrink C, Block M, et al. Effect of pacing chamber and atrioventricular delay on acute systolic function of paced patients with congestive heart failure. Circulation. 1999;99(23):2993-3001. doi:10.1161/01.CIR.99.23.2993

8. Kyriacou A, Rajkumar CA, Pabari PA, et al. Distinct impacts of heart rate and right atrial-pacing on left atrial mechanical activation and optimal AV delay in CRT. PACE - Pacing Clin Electrophysiol. 2018;41(8):959-966. doi:10.1111/pace.13401

9. Liang HY, Cheng A, Chang KC, et al. Influence of atrial function and mechanical synchrony on LV hemodynamic status in heart failure patients on resynchronization therapy. JACC Cardiovasc Imaging. 2011;4(7):691-698. doi:10.1016/j.jcmg.2011.02.019

10. SHEPPARD RC, REN J-F, ROSS J, McALLISTER M, CHANDRASEKARAN K, KUTALEK SP. Doppler Echocardiographic Assessment of the Hemodynamic Benefits of Rate Adaptive AV Delay During Exercise in Paced Patients with Complete Heart Block. Pacing Clin Electrophysiol. 1993;16(11):2157-2166. doi:10.1111/j.1540-8159.1993.tb01021.x 
11. FREEDOM - A Frequent Optimization Study Using the QuickOpt Method. https://clinicaltrials.gov/ct2/show/results/NCT00418314. Accessed August 11, 2020.

12. Ellenbogen KA, Gold MR, Meyer TE, et al. Primary results from the smartdelay determined AV optimization: A comparison to other AV delay methods used in cardiac resynchronization therapy (SMART-AV) trial: A randomized trial comparing empirical, echocardiography-guided, and algorithmic atrioventr. Circulation. 2010;122(25):2660-2668. doi:10.1161/CIRCULATIONAHA.110.992552

13. Whinnett ZI, Nott G, Davies JERR, et al. Maximizing efficiency of alternation algorithms for hemodynamic optimization of the AV delay of cardiac resynchronization therapy. PACE - Pacing Clin Electrophysiol. 2011;34(2):217225. doi:10.1111/j.1540-8159.2010.02933.x

14. Zweerink A, Salden OAE, van Everdingen WM, et al. Hemodynamic Optimization in Cardiac Resynchronization Therapy: Should We Aim for dP/dtmax or Stroke Work? JACC Clin Electrophysiol. 2019;5(9):1013-1025. doi:10.1016/j.jacep.2019.05.020

15. Brignole M, Auricchio A, Baron-Esquivias G, et al. 2013 ESC Guidelines on cardiac pacing and cardiac resynchronization therapy. Eur Heart J. 2013;34(29):2281-2329. doi:10.1093/eurheartj/eht150

16. Fang F, Sanderson JE, Yu CM. All Heart Block Patients with a Pacemaker Indication Should Receive Biventricular Pacing: One Move, Double the Gains? Circ Arrhythmia Electrophysiol. 2015;8(3):722-728.

doi:10.1161/CIRCEP.114.000626

17. Sharma PS, Vijayaraman P, Ellenbogen KA. Permanent His bundle pacing: shaping the future of physiological ventricular pacing. Nat Rev Cardiol. 2020;17(1):22-36. doi:10.1038/s41569-019-0224-z

18. Salden FCWM, Kutyifa V, Stockburger M, Prinzen FW, Vernooy K. Atrioventricular dromotropathy: Evidence for a distinctive entity in heart failure with prolonged PR interval. Europace. 2018;20(7):1067-1077. doi:10.1093/europace/eux207

19. Zanon F, Ellenbogen KA, Dandamudi G, et al. Permanent His-bundle pacing: A systematic literature review and meta-analysis. Europace. 2018;20(11):18191826. doi:10.1093/europace/euy058

20. Zweerink A, De Roest GJ, Wu L, et al. Prediction of Acute Response to Cardiac Resynchronization Therapy by Means of the Misbalance in Regional Left Ventricular Myocardial Work. J Card Fail. 2016;22(2):133-142. doi:10.1016/j.cardfail.2015.10.020

21. Vecera J, Penicka M, Eriksen M, et al. Wasted septal work in left ventricular dyssynchrony: A novel principle to predict response to cardiac resynchronization therapy. Eur Heart J Cardiovasc Imaging. 2016;17(6):624-632.

doi:10.1093/ehjci/jew019 
22. Vernooy K, Verbeek XAAM, Peschar M, et al. Left bundle branch block induces ventricular remodelling and functional septal hypoperfusion. Eur Heart J. 2005;26(1):91-98. doi:10.1093/eurheartj/ehi008

23. Delhaas T, Arts T, Prinzen FW, Reneman RS. Regional fibre stress-fibre strain area as an estimate of regional blood flow and oxygen demand in the canine heart. $J$ Physiol. 1994;477(3):481-496. doi:10.1113/jphysiol.1994.sp020209

24. Russell K, Eriksen M, Aaberge L, et al. A novel clinical method for quantification of regional left ventricular pressurestrain loop area: A non-invasive index of myocardial work. Eur Heart J. 2012;33(6):724-733. doi:10.1093/eurheartj/ehs016

25. Galli E, Leclercq C, Hubert A, et al. Role of myocardial constructive work in the identification of responders to CRT. Eur Hear J - Cardiovasc Imaging. 2017;(July):1-9. doi:10.1093/ehjci/jex191

26. Aalen J, Storsten P, Remme EW, et al. Afterload Hypersensitivity in Patients With Left Bundle Branch Block. JACC Cardiovasc Imaging. 2018. doi:10.1016/j.jcmg.2017.11.025

27. Massoullié G, Chouki C, Mulliez AA, et al. Effect of Optimization of Medical Treatment on Long-Term Survival of Patients With Heart Failure After Implantable Cardioverter Defibrillator and Cardiac Resynchronization Device Implantation (from the French National EGB Database). Am J Cardiol. 2018;121(6):725-730. doi:10.1016/j.amjcard.2017.12.013

28. Damy T, Ghio S, Rigby AS, et al. Interplay between right ventricular function and cardiac resynchronization therapy: An analysis of the care-HF trial (Cardiac resynchronization-heart Failure). J Am Coll Cardiol. 2013;61(21):2153-2160. doi:10.1016/j.jacc.2013.02.049

29. Ricci F, Mele D, Bianco F, Bucciarelli V, De Caterina R, Gallina S. Right heartpulmonary circulation unit and cardiac resynchronization therapy. Am Heart J. 2017;185:1-16. doi:10.1016/j.ahj.2016.11.005

30. Storsten P, Aalen JM, Boe E, et al. Mechanical Effects on Right Ventricular Function From Left Bundle Branch Block and Cardiac Resynchronization Therapy. JACC Cardiovasc Imaging. 2020;13(7). doi:10.1016/j.jcmg.2019.11.016

31. Sljivic A, Kleut MP, Bukumiric Z, Celic V. Association between right ventricle twoand three-dimensional echocardiography and exercise capacity in patients with reduced left ventricular ejection fraction. PLoS One. 2018;13(6):1-14. doi:10.1371/journal.pone.0199439

32. Arora S, Aarones M, Aakhus S, et al. Peak oxygen uptake during cardiopulmonary exercise testing determines response to cardiac resynchronization therapy. J Cardiol. 2012;60(3):228-235. doi:10.1016/j.jjcc.2012.03.004

33. Lumens J, Tayal B, Walmsley J, et al. Differentiating Electromechanical from 
Non-Electrical Substrates of Mechanical Discoordination to Identify Responders to Cardiac Resynchronization Therapy. Circ Cardiovasc Imaging. 2015;8(9):1-12. doi:10.1161/CIRCIMAGING.115.003744

34. Park HE, Chang S-A, Kim H-K, et al. Impact of Loading Condition on the 2D Speckle Tracking-Derived Left Ventricular Dyssynchrony Index in Nonischemic Dilated Cardiomyopathy. Circ Cardiovasc Imaging. 2010;3(3):272-281. doi:10.1161/CIRCIMAGING.109.890848

35. Lyon A, Dupuis LJ, Arts T, et al. Differentiating the effects of beta-adrenergic stimulation and stretch on calcium and force dynamics using a novel electromechanical cardiomyocyte model. Am J Physiol Circ Physiol. 2020. doi:10.1152/ajpheart.00275.2020

36. Dupuis LJ, Lumens J, Arts T, Delhaas T. Mechano-chemical Interactions in Cardiac Sarcomere Contraction: A Computational Modeling Study. PLoS Comput Biol. 2016;12(10):1-20. doi:10.1371/journal.pcbi.1005126

37. Niederer SA, Smith NP. Using physiologically based models for clinical translation: predictive modelling, data interpretation or something in-between? J Physiol. 2016;594(23):6849-6863. doi:10.1113/JP272003

38. Niederer SA, Lumens J, Trayanova NA. Computational models in cardiology. Nat Rev Cardiol. 2019;16(2):100-111. doi:10.1038/s41569-018-0104-y

39. Lee AWC, Costa CM, Strocchi M, Rinaldi CA, Niederer SA. Computational Modeling for Cardiac Resynchronization Therapy. J Cardiovasc Transl Res. 2018;11(2):92-108. doi:10.1007/s12265-017-9779-4

40. Arts T, Bovendeerd PH, Prinzen FW, Reneman RS. Relation between left ventricular cavity pressure and volume and systolic fiber stress and strain in the wall. Biophys J. 1991;59(1):93-102. doi:10.1016/S0006-3495(91)82201-9

41. Pluijmert M, Lumens J, Potse M, Delhaas T, Auricchio A, Prinzen FW. Computer modelling for better diagnosis and therapy of patients by cardiac resynchronisation therapy. Device Ther. 2015;4(1):62-67. doi:10.15420/aer.2015.4.1.62

42. Arts T, Lumens J, Kroon W, Delhaas T. Control of whole heart geometry by intramyocardial mechano-feedback: A model study. PLoS Comput Biol. 2012;8(2). doi:10.1371/journal.pcbi.1002369

43. Kerckhoffs RCP, Omens JH, Mcculloch AD. Mechanical discoordination increases continuously after the onset of left bundle branch block despite constant electrical dyssynchrony in a computational model of cardiac electromechanics and growth. 2012:65-72. doi:10.1093/europace/eus274

44. Corral-Acero J, Margara F, Marciniak M, et al. The 'Digital Twin' to enable the vision of precision cardiology. Eur Heart J. 2020:1-11.

doi:10.1093/eurheartj/ehaa159 
45. NHR Rapportage 2019;; 2019.

https://nederlandsehartregistratie.nl/wp-content/uploads/2020/01/NHR-

Rapportage-2019-per-spread-230120.pdf.

46. Brignole M, Auricchio A, Baron-Esquivias G, et al. 2013 ESC Guidelines on cardiac pacing and cardiac resynchronization therapy: Addenda The Task Force on cardiac pacing and resynchronization therapy of the European Heart Rhythm Association(EHRA). Eur Heart J. 2013;ESC Guidel:1-21.

doi:10.1093/eurheartj/eht180

47. Viceconti M, Pappalardo F, Rodriguez B, Horner M, Bischoff J, Musuamba Tshinanu F. In silico trials: Verification, validation and uncertainty quantification of predictive models used in the regulatory evaluation of biomedical products. Methods. January 2020. doi:10.1016/j.ymeth.2020.01.011 


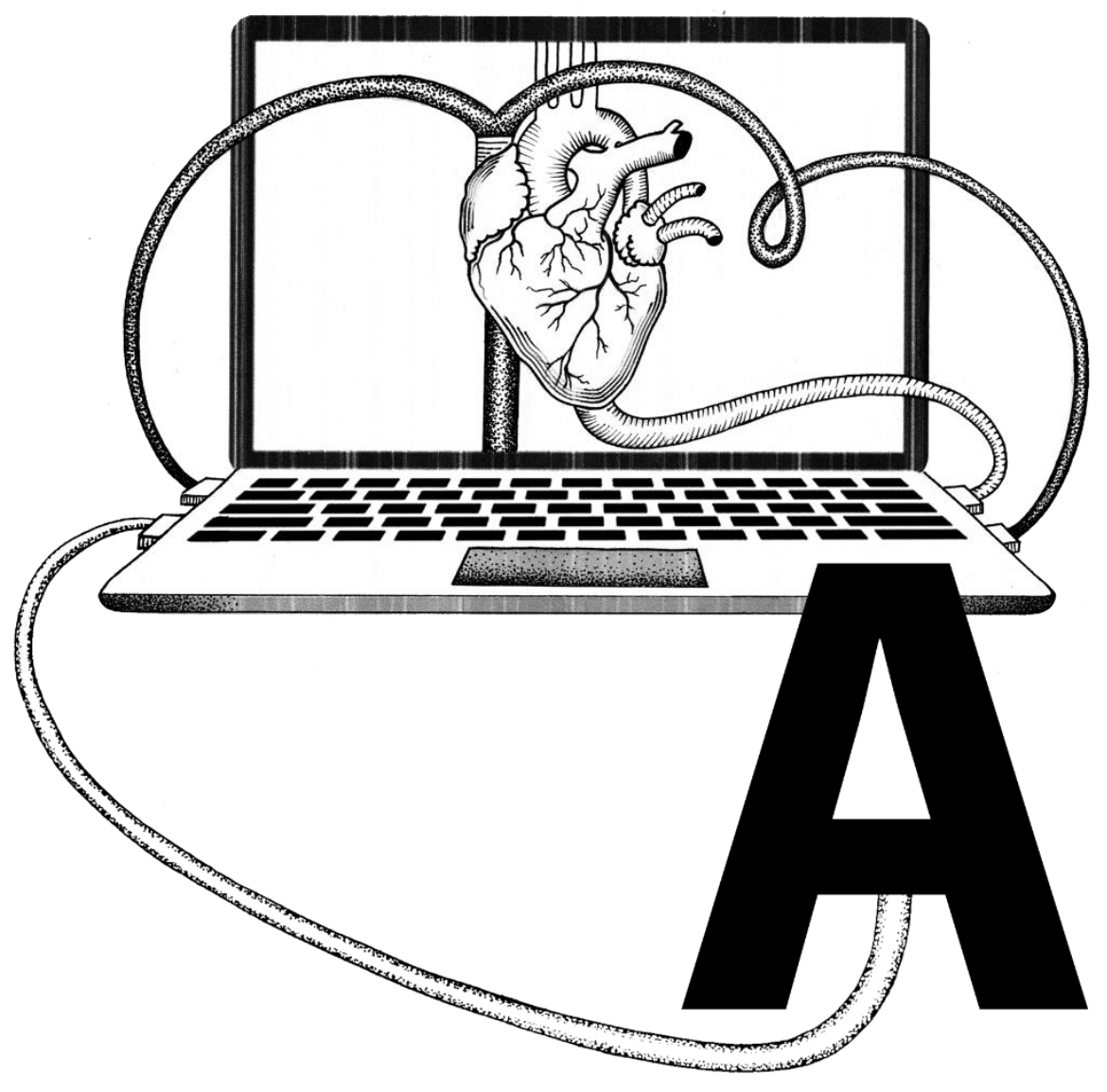




\section{Appendix A}

Summary 

The pumping action of the healthy human heart ensures circulation of blood through our cardiovascular system. Normal cardiac pump function is by no means self-evident, but requires all four chambers to work together in a continuous and tightly coordinated effort. Cardiac muscle tissue and thereby the heart contracts when it is triggered with an electrical stimulus. Disturbances in conduction of this electrical stimulus can result in impaired cardiac function and thereby insufficient blood circulation.

When disturbances in electrical conduction lead to symptoms, artificial electrical stimulation of the heart using a pacemaker may (partially) restore a patients' electrical activation. One or more electrodes are placed in the atria and/or ventricles to provide an electrical stimulus. In this thesis, we aim to improve mechanistic understanding of how the variable aspects of the delivery of pacemaker therapy influence cardiac pump function. Where most studies primarily focus on left ventricular (LV) function we used a whole-heart approach to better understand how cardiac pacing therapy can be optimized and selection of patients receiving pacing can be improved. For this whole-heart assessment we used the CircAdapt computer model to study interactions between the heart chambers and between the heart and surrounding circulation. CircAdapt is a lumped parameter computer model of the heart and circulation that enables real-time beat-to-beat simulations of the mechanics and hemodynamics of the human cardiovascular system under physiological and pathophysiological circumstances.

In a healthy heart, the atrioventricular (AV) node is the only location where electrical signals can propagate from the atria to the ventricles. In the $A V$ node the electrical wave front propagation is slower than in cardiac tissue, causing a delay between atrial and ventricular activation, which is essential for ventricular filling. Cardiac pacing can be employed to restore AV coupling in patients with a blockage in the AV node. In

Chapter 2 we used CircAdapt computer simulations to find the optimal AV delay at various inter-atrial delays, ventricular pacing sites and heart rates. The simulations showed that an increase in heart rate leads to a shift of optimal pump function to shorter AV delays. In contrast, the optimal AV delay increases with increasing interatrial activation delay and with decreasing interventricular activation delay (such as during 
biventricular rather than right ventricular (RV) or LV pacing). The latter observations suggest that both LV and RV filling dynamics determine the optimal AV delay. Simulations suggested that the effective wholeheart AV delay as percentage of the cardiac cycle length, incorporating activation times of both the left and right heart, enabled a more consistent definition of AV coupling.

Blockage of parts of the ventricular rapid conduction system, such as the left and right bundle branch, can lead to dyssynchronous ventricular activation and a less efficient contraction. Cardiac resynchronization therapy (CRT) can be applied in patients with ventricular dyssynchrony to obtain a more synchronous activation by pacing both ventricles. In $\mathrm{CRT}$, besides the AV delay, the timing between ventricular contractions can also be affected by the ventriculo-ventricular (VV) delay of the pacemaker. In Chapter $\mathbf{3}$ we combined animal-experimental data and computer simulations, covering a wide range of $A V$ and $V$ delay settings, and demonstrated that the LV and RV respond in an opposite manner to variations in LV or RV pacing delays, with RV contractility being optimal during RV pre-excitation and LV contractility being optimal during LV pre-excitation. On the other hand, simulated cardiac output data showed optimal values during (almost) simultaneous RV and LV pacing, suggesting that overall cardiac pump function depends on both left and right heart function. In Chapter $\mathbf{4}$ we showed the importance of ventricular dyssynchrony during pacing for outcome after CRT in patients and computer simulations. We demonstrated that the presence of significant LV dyssynchrony during pacing is associated with lack of LV reverse remodeling in CRT patients.

The benefit of CRT not only depends on the settings of the pacemaker but also on the condition of the patient's heart. Echocardiographic measurements of regional ventricular deformation and myocardial work may be used to improve the selection of CRT candidates. 
In Chapter 5 we demonstrated that the abnormal distributions of myocardial deformation and work that exist during dyssynchronous ventricular activation are amplified when the same electrical dyssynchrony coincides with a higher loading (afterload) of the LV. Afterload is thus an important additional variable that should be known for proper interpretation of ventricular deformation data.

A further study in this thesis extents animal experimental and clinical work of others, showing that prediction of CRT response can further be improved by not only measuring LV deformation but also RV deformation. As shown in Chapter $\mathbf{6}$ a decrease in RV contractile reserve decreases the CRT response. Noninvasive measurement of RV deformation can help to identify patients with impaired RV function and thereby improve patient selection for CRT.

The work presented in this thesis demonstrates that CircAdapt can be a valuable tool to unravel the complex mechanical and hemodynamic interactions that play a role in the working mechanism of cardiac pacing therapy. The combination of computer simulations with animal experimental and clinical data showed that measurements providing insight in whole-heart activation are important for determining the patient's potential for positive response to cardiac pacing. Measurement of LV deformation alone provides incomplete insight into ventricular electrical dyssynchrony, since right ventricular function and LV afterload also modulate the mechanical discoordination in dyssynchronous hearts. Therefore, an integrative whole-heart assessment is the key to achieve optimal response in patients receiving cardiac pacing therapy. 


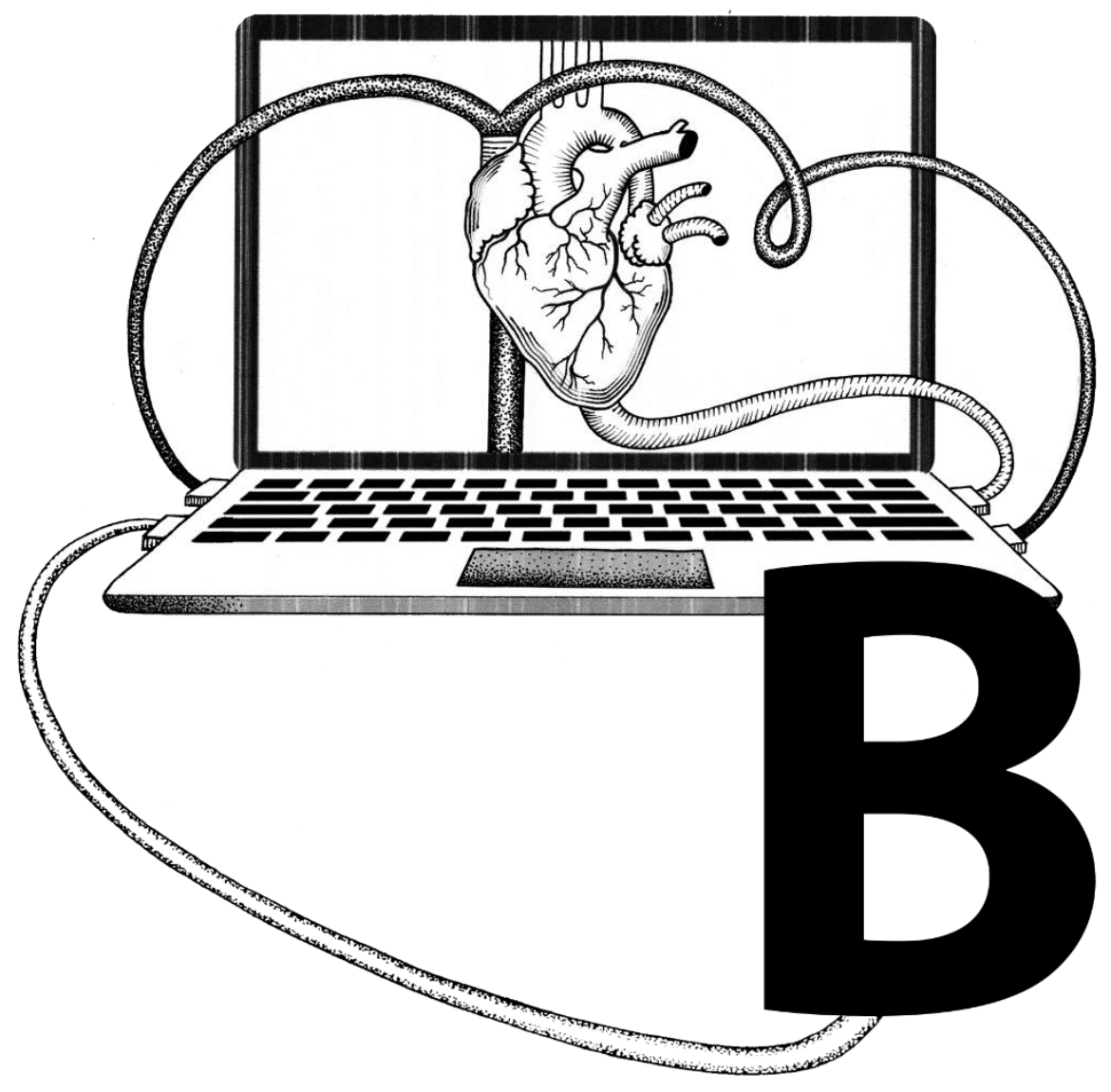


Appendix B

Acknowledgment Dankwoord 

In dit leven is het lastig om in je eentje dingen voor elkaar te krijgen, hoe graag ik dat zelf soms ook zou willen (en probeer). Zonder hulp en aanwijzingen van velen was het nooit gelukt om aan deze PhD te beginnen, laat staan het af te maken. Maar hier ben ik dan toch het dankwoord aan het typen. Om te beginnen wil ik iedereen die het mogelijk heeft gemaakt op dit moment te komen, met mijn hele hart bedanken.

De PhD en daarmee de bijzondere tijd die dit opgeleverd heeft waren niet mogelijk geweest zonder Joost en Frits. Dankzij het project waar ik in heb kunnen werken, werd het mogelijk mijn PhD te doen en dankzij jullie was het ook mogelijk iets te maken wat uiteindelijk geresulteerd heeft in deze thesis. Joost, doordat we op een leuke manier over de (vaak gesimuleerde) fysiologie konden praten ontstonden er veel gedachtestromen waarvan (helaas?) slechts een klein deel opgeschreven is. Ook is het dankzij jou mogelijk geweest op verschillende plekken buiten Maastricht hierover te praten, iets wat zonder jou niet mogelijk was geweest. Dit samen met het biertje, wijntje en diner af en toe maakte het tot een gezellige leuke periode! Frits, jij was een belangrijke schakel waardoor soms weer nieuwe ideeën ontstonden, maar waardoor vaak ook onderdelen wel in deze thesis terecht gekomen zijn. Naast de inhoudelijke gesprekken zullen vooral de gezellige avonden mij bijblijven.

Tammo, bedankt voor de feedback die je gegeven hebt. Hoewel er een enkele keer een generator geregeld moest worden, was het met een paar biertjes erbij vaak gezellig.

\section{Prof. Schotten, dr. Allaart, prof. Crijns, prof. Peeters en dr.} Westerhof, leden van de beoordelingscommissie, wil ik bedanken voor het kritisch lezen en beoordelen van mijn thesis.

Peter en John wil ik in het bijzonder bedanken voor het mij laten begrijpen hoe CircAdapt werkt, en later het ouwehoeren daarover en andere zaken (vaak met een paar biertjes). 
Lauren, Frank en Aurore wil ik bedanken voor de leuke tijd in Maastricht en op reisjes daarbuiten in deze periode. Lauren without you (especially in combination with Peter) Maastricht and time at BME would have been a whole lot more boring. You're probably the one that pulled me (and lots of others) along in the beginning, blaming people for planning ahead, how life can change...

Een leuke tijd op een afdeling heb je door de mensen! En een leuke afdeling was het, bedankt daarvoor Ben, Sjeng, Maarten, Raoul, Bennis, Letty, Myrthe, Anneloes, Koen, Wouter, Mehrdad, Koen, Shaiv, Niek, Pamir, Tim, Tijmen, Nick, Job en alle (andere) studenten door de jaren heen. Het liefst had ik dit bedanken gedaan bij een BBQ buiten op het gras of een borrel, toch wel de leukste after-work momentjes maar helaas niet mogelijk in het afgelopen jaar. Claudia, Melania, Ahmed, Afrah and Feddo I guess it's up to you (and the lot mentioned above to help you all) to keep BME a great department and Maastricht a good time. Claire bedankt voor het managen van heel BME en het regelen voor van alles en nog wat.

Floor en Luuk, dankzij jullie zat er toch nog een beetje een klinische kant aan het werk in Maastricht, maar was er ook een andere kant van de gang waar ik soms wat geroddel kon horen. Ook de andere mensen van de CRT meeting wil ik graag bedanken. Het was zeer leerzaam om te zien hoe vanuit verschillende kanten gekeken wordt naar pacing en te zien/horen wat er binnen en buiten Maastricht zoal gebeurt. Bedankt Frans, Kevin, Nienke, Meike, Moedi, HongXing, Philip, Richard, Rick, Twan, Uyen en de rest.

Bedankt Bjorn, Bart, Tom en Niels voor de top tijd in Enschede en daarna. Zonder jullie was het begin van mijn academische carrière niet geslaagd, of in ieder geval stukken minder leuk geweest. $\mathbf{5 0} \%$ Erik $\mathbf{1 0 0 \%}$ awesome wil ik op voor hetzelfde bedanken iets later in Enschede en daarna.

Ad bedankt voor het aanwakkeren van mijn interesse in de cardiologie. Tiny bedankt voor alles door de jaren heen, als ik op vrijdag in Hengelo ben, weet ik wie ik moet appen. Ton, bedankt voor al je adviezen. 
Luuk en Jim bedankt dat jullie mijn paranimfen willen zijn voor deze gelegenheid maar vooral bedankt dat jullie mijn broers (naja broertjes) zijn... Ook bedankt voor het helpen met klussen/slopen in deze periode! Ma bedankt voor alles, van het praktische tot het motiveren... Kan niet altijd makkelijk zijn geweest en toch ben je altijd mijn Ma.

Bedankt Shannen, voor het tolereren van mijn gegodver als het toch weer eens tegen zat en het aanhoren van mijn gedram. Dankzij dit hele PhD avontuur ben ik je tegengekomen in Maastricht en alleen daarom al (zijn genoeg andere redenen) zou ik niet zo moeten godverren op de mindere momenten van het afronden ervan...

Pa, helaas kan je dit niet lezen. Toch wil ik ook jou bedanken voor alles. In dit avontuur was de nieuwsgierigheid en eigenwijsheid, die jij had en doorgegeven hebt, van grote waarde! 


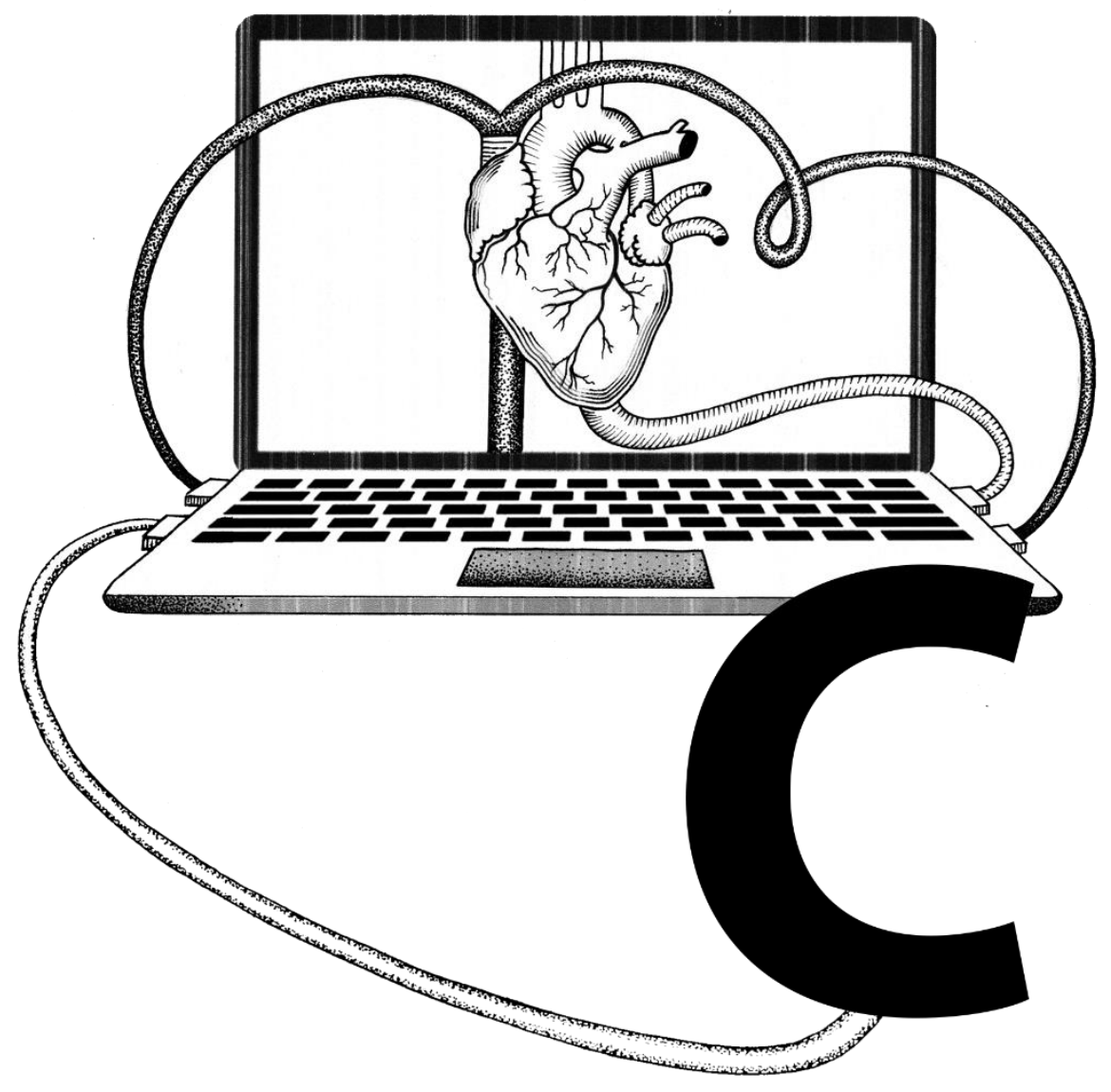


Appendix C

Curriculum Vitae 



\section{About the author}

Erik Willemen was born on January 10, 1989 in Hengelo, Overijssel, the Netherlands. After attending high school in Hengelo, he went to Enschede to study Technical Medicine. He obtained his bachelor degree in 2010, followed by his master degree in 2015.

During the clinical research internships in the Master's track 'Medical Signaling' he worked within the department of Urology, Pulmonary medicine and Internal medicine. During his studies he developed an interest for systems physiology which resulted in a graduation internship at the Laboratory of Clinical Cariovascular Physiology at the Amsterdam Medical Centre. Erik finished his master degree successfully with a thesis on the effect of body position (supine or upright) on the cardio- and cerebrovascular response to exercise.

Erik's academic career continued at the department of BioMedical Engineering at the Maastricht University with research focused on cardiac pacing. Starting out as a project within the PushCart project, computer simulations formed a important basis within this research. Further collaboration with the physiology and cardiology departments allowed the creation of a wholehearted computational assessment of cardiac pacing, presented in this thesis.

Erik will continue his career at the clinical neurophysiology department at the Canisius Wilhelmina Ziekenhuis, in Nijmegen.

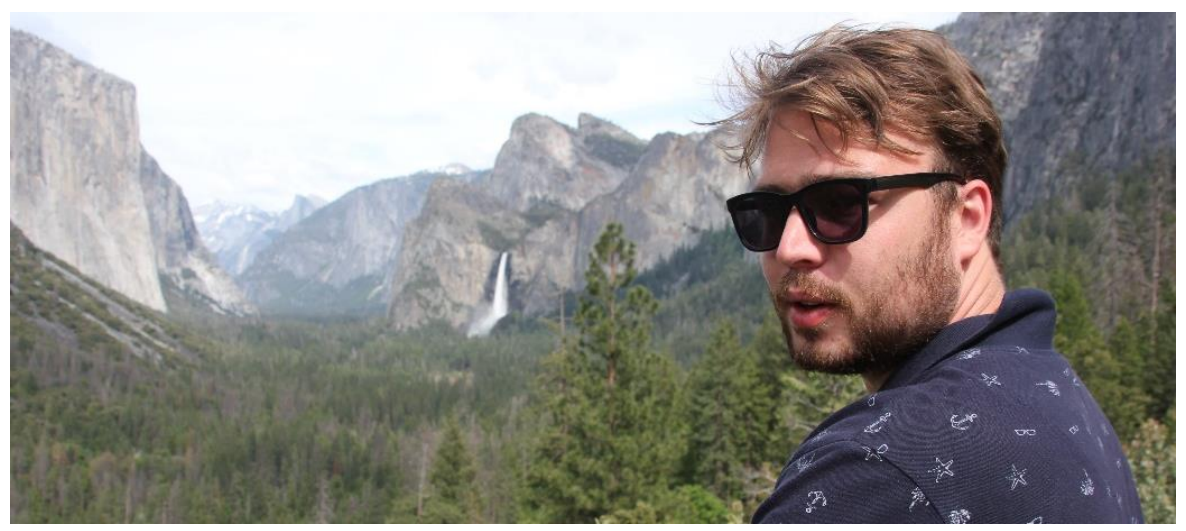




\section{List of publications}

\section{Peer reviewed journals}

Willemen E, Schreurs R, Huntjens PR, Strik M, Plank G, Vigmond E, Walmsley J, Vernooy K, Delhaas T, Prinzen FW and Lumens J (2019) The Left and Right Ventricles Respond Differently to Variation of Pacing Delays in Cardiac Resynchronization Therapy: A Combined Experimental-Computational Approach. Front. Physiol. 10:17.doi: 10.3389/fphys.2019.00017

Gauthey A, Willemen E, Lumens J, Ploux S, Bordachar P, Ritter P, Prinzen FW, Lejeune S, Pouleur AC, Garnir Q, Marchandise S, Scavée C, Wauters A, de Waroux JLP. Impact of paced left ventricular dyssynchrony on left ventricular reverse remodeling after cardiac resynchronization therapy. J Cardiovasc Electrophysiol. 2020 Jan 6. Doi: 10.1111/jce. 14330.

\section{Editorial comments}

Frits W. Prinzen, Erik Willemen, Joost Lumens, LBBB and High Afterload: A Dangerous Liaison? JACC: Cardiovascular Imaging; 10.1016/j.jcmg.2017.12.002

Lumens J, Willemen E, Prinzen FW. Does the right go wrong during cardiac resynchronization therapy? JACC Cardiovasc Imaging. 2020 Jan 16 


\section{Conference proceedings and (poster) presentations}

- Conference of Applied Modeling in Acute Care, Enschede, April 2017; Oral presentation: Difference in right and left ventricular response during cardiac resynchronization therapy (CRT): Insights from canine-specific simulations

- EHRA CardioStim 2017, Vienna, June 2017; Poster Presentation: Difference in right and left ventricular response during cardiac resynchronization therapy (CRT): insights from canine-specific simulations

- Myocardial Velocity and Deformation Imaging, Leuven, February 2018; Oral presentation: Linking load, deformation and pump function in dyssynchronous heart failure

- Dutch Heart Foundation PhD course, Papendal, October 2017; Poster presentation selected for Ted talk: Left-right ventricular interaction during cardiac resynchronization therapy (CRT) optimization

- World Congress of Biomechanics, Dublin, July 2018; Oral Presentation: Left-right ventricular interaction during cardiac resynchronization therapy (CRT) optimization

- EuroEcho-Imaging 2018, Milan December 2018, Young Investigator Award (runner-up), Oral Presentation): Afterload sensitivity of global versus septal wasted work ratio: implications for prediction of cardiac resynchronization therapy response

- EHRA 2019, Lisbon March 2019, Winner at the best oral ecardiology abstract award competition; HRS 2019, San Fransisco May 2019, Poster Presentation: Afterload dependence of left and right ventricular response to pacing delay optimization: a combined experimental-computational study 